$\begin{array}{ll}\text { Italique } & \text { Italique } \\ \text { Poésie italienne de la Renaissance }\end{array}$

VII | 2004

Varia

\title{
Una gestazione e un parto gemellare : la prima e la seconda parte dei Sonetti di Benedetto Varchi
}

\section{Giuliano Tanturli}

\section{(2) OpenEdition}

\section{Journals}

\section{Edizione digitale}

URL: http://journals.openedition.org/italique/128

DOI: $10.4000 /$ italique. 128

ISSN: 1663-4438

\section{Editore}

Librairie Droz

\section{Edizione cartacea}

Data di pubblicazione: 1 novembre 2004

Paginazione: 43-87

ISBN: 2-600-00976-0

ISSN: 1423-3983

\section{Notizia bibliografica digitale}

Giuliano Tanturli, « Una gestazione e un parto gemellare : la prima e la seconda parte dei Sonetti di Benedetto Varchi », Italique [Online], VII | 2004, online dal 05 octobre 2009, consultato il 01 mai 2019. URL : http://journals.openedition.org/italique/128; DOI : 10.4000/italique.128 


\author{
G I U L I A N O TAN T UR L I \\ U N A G E T A Z I O N E \\ E UN PARTO GEMELLARE : \\ LA PRIMA E LA SECONDA PARTE \\ DEI SONETTI \\ DI B ENEDETTO VARCHI
}




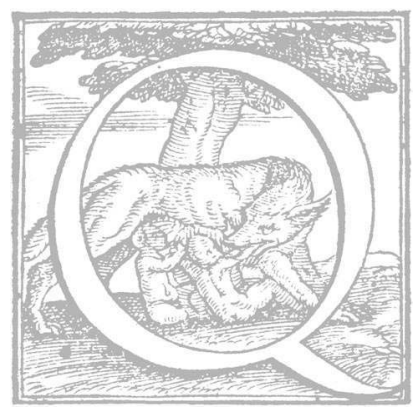

Uesto contributo vorrebbe ricercare e descrivere come Benedetto Varchi (Firenze I503Is65) riuni e dispose un gran numero di sonetti suoi e di corrispondenti, mandati a stampa in due parti nel I555 e nel I557; potrà classificarsi come studio filologico d'una struttura. In quanto struttura il duplice libro, difficile per l'autore da ordinare e per noi da studiare a causa anche della mole, è di non poco interesse proprio per il contrasto fra ricerca d'unità e necessario sdoppiamento e ancor più per la ragione di quel contrasto. Da un canto tira il collaudato schema, a cui non si vorrebbe rinunciare per la garanzia d'organica unità, del "canzoniere" secondo il modello primo del Petrarca, di libro, però, se non intimo, in cui prevale lo sguardo rivolto a sé; dall'altro c'è la natura epistolare della maggior parte dei sonetti, quindi rivolta all'esterno, ai multiformi rapporti sociali d'un grande e autorevole letterato.

Nel I55s, dunque, Benedetto Varchi stampava la prima parte dei sonetti, De sonetti di m. Benedetto Varchi, Parte prima., Con Privilegio. In Fiorenza apresso m. Lorenzo Torrentino. MDLV ( $\mathrm{t} 5 \mathrm{5})$, con una dedica a «don Francesco Medici principe di Firenze», datata «d'Orvieto la vigilia del Corpus Domini dell'anno MDLV》, cioè il I2 gingno (a Orvieto l'autore doveva trovarsi in visita a monsignor Lorenzo Lenzi, il suo "Lauro»). ${ }^{1}$ La stessa silloge senza varianti di rilievo, nessuna nell'ordine e nelle presenze, e aggiunte in fine tre ecloghe, comparve lo stesso anno col titolo I sonetti $\mathrm{di} \mathrm{m}$. Benedetto Varchi, novellamente messi in luce., in Venetia per Plinio Pietrasanta. MDLV, e con dedica a firma di Giorgio Benzoni a Giovanni della Casa, datata "Di Vinegia il primo di luglio MDLV》. Le due edizioni, quindi, furono di fatto contemporanee. Il Benzoni cosi delimita l'ufficio suo: l'autore «s'è contento ch'altri dedichi quel ch'a lui si conveniva dedicare; perciò che, sendogli io quello strettissimo amico, che io sono, m'b commesso che io ponga studio che l'opera sua si ricoveri sotto l'ombra d'un signore che per sé il vaglia et che sia amicissimo suo; et senza specificar altramenti il desiderio che aveva, ha lasciato che da me ne faccia elettione». Dunque anche di questa stampa l'iniziativa spetta al Varchi, che incaricò il Benzoni di stendere la prefazione per un personaggio di valore e amicissimo suo. É ovvio che l'incaricato si dovesse premunire d'averne centrato il desiderio, se, come forse è altrettanto ovvio, non gli era stato rivelato. Le due dediche all'erede del duca Cosimo e al nuovo segretario di stato di Paolo IV, interprete e artefice d'una politica antimperiale e pertanto antimedicea, seguendo in cio i propri sentimenti d'esule volontario, sono in patente opposizione; ma consone a un Varchi antico repubblicano, che era dovuto venire a patti col duca e accettare gli obblighi 
conseguenti, senza tuttavia rinnegare il proprio passato e gli amici che, diversamente da lui, in patria non erano dovuti tornare. Ma non di questo, che era giusto richiamare, si vuole ora discorrere, se non per avvertire che una dedica era, come l'autore scrive in quella a sua firma, novità estranea all'"esempio dei migliori e più gravi autori, che rime habbiano composto e mandato fuorì, cioè, specificava, il Petrarca e il Bembo (t5 5, c. * IIr-v). E già questo mette in tutta evidenza la proiezione sociale anche del libro nel suo insieme. Che le dediche siano due, con due conseguenti e separate stampe, mostra come $i$ rapporti nei quali esso si colloca siano anche divergenti.

Alla prima parte dei sonetti segui due anni dopo presso lo stesso stampatore fiorentino, e solo presso di lui, la seconda: De' sonetti di $\mathrm{m}$. Benedetto Varchi colle risposte, e proposte di diversi parte seconda., in Fiorenza appresso Lorenzo Torrentino MDLVII ( 5 5 7). Le dueparti coordinate nei titoli, che ne fanno due eventi d'un solo progetto, si presentano come libri d'aspetto diverso, se non di diversa natura. Più semplice e breve dire del secondo, che è un epistolario poetico diviso in due sezioni da una carta bianca: settantanove proposte del Varchi ciascuna con la sua risposta; centoquarantaproposte d'altri a lui e la sua risposta; un tipo di libro poetico non comune, per il quale non mi soccorrono precedenti. Si comincia con quattro scambi di sonetti con Annibal Caro su Lorenzo Lenzi Lauro, di cui il Caro fu precettore, e sull'amore del Varchi per lui. Sulla soglia della seconda parte, cosi, s'affaccia l'ombra di «Quella pianta» (par. $2^{a}$ I 3), che subito dopo entra in scena in prima persona, rispondendo ai sonetti V e VI. ${ }^{2} \mathrm{Ne}$ segue uno a Schiatta Bagnesi e risposta ancora sull'amore, anzi, mi pare, su questo principale amore, a chiudere l'inizio appunto nel suo nome; che spesso e variamente ritornerà nelle corrispondenze. Entro le due sezioni le coppie sono riunite per corrispondenti in serie anche abbastanza lunghe, come quelle con Lelio Bonsi, da XIV a XIX e da CXXXIV a CXLI, e con Lucio Oradini, da XX a XXV e da CXLII a CXLVIII. Ė ovvio anche perché queste due corrispondenze si susseguano nell'una e nell' altra sezione: $i$ due sono $i$ discepoli prediletti del Varchi a partire dai tardi anni Quaranta, introdotti giovanissimi nell' Accademia Fiorentina (vi lessero pubblicamente nel' 49, il Bonsi, e nel'5o, l'Oradini). ${ }^{3}$ Talvolta le successioni sono indotte dalla parentela dei corrispondenti: per esempio, la corrispondenza con Luigi Alamanni, LIX-LX, e col figliolo Battista, LXI-LXIII, che nella seconda sezione resteranno uniti, scambiandosi di posto: prima Battista, CV-CVII, poi ilpadre, CVIII-CX; o dal casato: Ludovico, LXXX, Vincenzio, LXXXI, Niccolo, LXXXII, Ugolino Martelli, LXXXIII-LXXXVII, che aprono la seconda sezione. Di questi solo Ugolino compariva nella prima, x, chiuso fra due Strozzi, Giovambattista, IX, e Carlo, XI-XII: delprimo è evocato un soggiornopadovano; il secondo lo condivise con lo stesso Ugolino nel I540, col quale dal I548fu a Roma al seguito del cardinale Ridolfi, sicché fra i duepersonaggi è stato rilevato un percorso in parte parallelo. ${ }^{4}$ Oltre a queste affinità fra $i$ corrispondenti c'è l'affinità di 
materia della corrispondenza X (con Ugolino Martelli) e XI (con Carlo Strozzi), di compianto e conforto funebre. $E$ altre volte, naturalmente, gli scambi di sonetti sono riuniti dall'argomento, come quello inviato da Simone della Volta, CXV, posto dietro $i$ CXI-CXIV del Bronzino, trattando tutti dell'invidia che s'abbatté sul Varchi. Talvolta il legame sarà un'esplicita o implicita presenza: LXXV (a Bernardo Cappello), LXXVI (a Domenico Venier), LXXVII (a Giovanni della Casa), tutti parlano del Bembo. Dall'ultima di queste corrispondenze si passa opportunamente a quella con Francesco Nasi (LXXVIII), nella quale si parla del Casa, suggerendo al Nasi di ricordargli un passaggio d'un sonetto giustappunto del Bembo.' Il legame può esser anche formale. Fra l'ultimo scambio di sonetti con Giovambattista Busini, CIV, e il primo con Battista Alamanni, CV, è comune la rima A con un intero secondo emistichio, «seconda morte» (CIV risposta, 4, e CV proposta 4); gli incipit di CIV proposta e risposta, Arsi con dura e insopportabil sorte, Ben riconosco in voi quel saggio e forte, sono variamente echeggiati e come riassunti da quello della proposta di CV, Ben contender mi può l'empia mia sorte. La divisione formale e oggettiva fra $i$ sonetti di proposta e di risposta del Varchi viene talvolta a separare dialoghi di per sé in continuità: $i$ sonetti LXXI, a Antonio Landi, e LXXII-LXXIII, a Bernardo Davanzati, con le risposte, su un soggiorno nella villa della Tana del primo, posti verso la fine della prima sezione, trovano il loro seguito nei due ultimi della seconda, CCXVIII, del Landi e risposta, sul Monte Senario, il luogo dell'amore e della poesia del Varchi, che era stato introdotto in LXXI, come l'unico superiore alla Tana, e CCXIX del Davanzati e risposta in lode di questa villa. D'altra parte, richiamandosi quelle corrispondenze con tutta chiarezza e essendo poste in parallelo, la dislocazione, causata dalla circostanza che vede il titolare del libro prima proponente quindi risponditore, assume un esplicito valore architettonico.

La prima parte dei sonetti del 's's ha un'apertura retrospettiva al modo del Petrarca, esemplata su quella del Bembo:

Quel ch'Amor mi dettò casto e sincero

D'un Lauro verde ne' miei più freschi anni

Cantai colmo di gioia e senza inganni,

Se non leggiadro, almen felice e vero.

Febo, che puoi sol dar condegno e 'ntero

Pregio e ristoro alle fatiche e a i danni

Di quell'alme innocenti che coi vanni

Volano al ciel del loro ingegno altero,

Ch'io viva no, ma ben ti prego humile,

Se mai per te soffersi o freddi o fami,

Che non del tutto mi disfaccia morte; 
E quei più d'altri mai ben colti rami

Della tua pianta e mia con nuova sorte

Fioriscan sempre in rozzo e secco stile.

Del primo sonetto delle Rime di Pietro Bembo è condivisa la rima $B$ e tre delle parole in rima, che sono anche $(A)$ del primo dei Sonetti e canzoni di Iacobo Sannazaro e provengono dal Petrarca, Rerum vulgarium fragmenta 60 e 357. Del Bembo è il generale schema, che più proemiale non si potrebbe: argomento, I-4, invocazione, 5-I4, con identica mossa, «Febo, che puoi», da «Dive, per cui»; come identica è anche quella della seconda terzina, «E quei più d'altri», da «E quella strada». Del Bembo, I, è il «Cantai» (3); ma non n'è tolto il «Piansi», anzi, c'è il suo contrario: "Cantai colmo di gioia e senza inganni», come impone la natura omosessuale dell'amore del Varchi, che non puo mostrare $i$ tratti della passione, ma deve ammantarsi di quelli nobilitanti e disinteressati dell'amicizia. Proemiale anche "Quel ch'Amor mi dettò», da Ov. Am. II I 38: "Carmina, purpureus quae mibi dictat Amor).

Come c'è una così evidente apertura di libro, di libro di poesia lirico-elegiaca, che noi abbiamo l'abitudine di chiamare per comoda convenzione canzoniere, altrettanto esplicita, anzi dichiarata è la funzione di chiusura dell'ultimo, DXXXIV:

Da voi felice e senza alcuno affanno

Hebbe principio il mio cantare et hora

Felice e lieto in voi fornisce ancora,

Arbor del sole, al ventottesimo anno;

Ma le sante radici, che mi stanno

E stetter dentro al cor sì dolci, ognhora

In mezzo l'alma (o viva il corpo o mora)

Fibre maggiori e più profonde havranno.

Per voi de la comune schiera fuore

Uscii, pianta del ciel, per voi mi volsi

All'erta, e la seguii, strada d'honore.

Altro che voi né chiesi mai né volsi

Né voglio o cheggio infino all'ultime hore,

Che ben fin fa chi bene amando muore.

Quanto la chiusa di questo libro di sonetti è dichiarata e esplicita, tanto, e di conseguenza, poco bisogno ha, per avvertire che si «fornisce», di essere scopertamente canonica. Alla fine più canonica dal Petrarca (Rvf. 364-366) al Bembo (Rime CLXIII-CLV), domani al Casa (Rime LXIV) era e sarà una o più preghiere. In verità nemmeno questo chiudere facendo il punto e computo 
cronologico, di cui il Varchi si contenta, manca della debita autorizzazione del Petrarca, Rvf. 364, I-4: "Tennemi Amor anni ventuno ardendo, / Lieto nel foco, e nel duol pien di speme; / Poi che madonna e'l mio cor seco inseme / Saliro al ciel, dieci altri anni piangendos; e subito dopo questo consuntivo, dal v. 5 del sonetto, la contrizione e le preghiere. Quindi della chiusa secondo $i$ Rerum vulgarium fragmenta il Varchi si ferma al primo atto, non si scosta. Meno esemplare e rilevato il computo degli anni nel XCVIII dei Sonetti e canzoni del Sannazaro: "Giungendo al fin del sestodecim'anno» (IO), che se non è l'ultimo testo della stampa del I530, è seguito solo dai tre capitoli, ben separati dal resto, quasi un'appendice, se non altro metricamente, e da una pagina bianca (intercalata anche fra capitolo e capitolo). Un accenno di preghiera, a parte i sonetti spirituali posti all'interno, c'è, nel libro del Varchi, ma in principio, a II, I-8: "Alsi et arsi gran tempo e fu l'algore / E l'ardor cosi dolce e cosi santo, Che quel ch'a gl'altri suol vergogna e pianto, / A me sempre portò gioia et honore. I A te vero del cielo, alto Fattore / E della terra, sia la gloria e'l vanto, / E a voi, cui sole adoro al mondo e canto, / Frondi, degno del sol pregio et amore». Le preghiere in fine dei Rerum vulgarium fragmenta, delle Rime del Bembo sono preghiere che chiedono perdono o intercessione (Vergine bella) e rispondono al senso di vergogna e pentimento del consuntivo proemiale (Rvf. I, 9-I4; Bembo, Rime I, 9-I4). Posto che l'amore omosessuale del Varchi non può proporsi che come pura amicizia, santa e nobilitante, la preghiera non poteva essere che di ringraziamento, quale è quella di II; anticipata in principio forse proprio per chiarire sotto quest'aspetto non l'adeguamento, ma l'opposizione ai modelli. Il richiamo al principio dall'epilogo c'è anche nel Varchi: nel ribadire la felicità e la serenità del suo amore; ma anche, più sottilmente, in un dato formale. S'è visto che tre delle parole in rima A di I sono in rima, rispettivamente $B$ e A, nei sonetti proemiali del Bembo e del Sannazaro; la quarta, affanni, non si perde, è serbata a aprire, declinata al singolare e preceduta da preposizione privativa, l'epilogo e chiudere cosi circolarmente il tutto.

La prima parte dei sonetti si presenta, dunque, nell'inizio e fine che la delimitano e definiscono come libro organico di poesia amorosa, dell'amore che ora si diceva, per il Lauro o (DXXXIV) «Arbor del sole», nella cronaca Lorenzo Lenzi. La presenza d'un senhal, oltre che di questo senhal, che volge al maschile quello del Canzoniere, lo riporta all'archetipo piu di altri esemplari cinquecenteschi, che, e fra questi quello di Pietro Bembo, non sempre ce l'hanno. La chiusa sembrerebbe alludere anche a un percorso forse non rettilineo e, certo, a un flusso temporale: «Da voi [...] Hebbe principio il mio cantare et hora [...] in voi fornisce [...] al ventottesimo anno» (DXXXIV, I-4), il cui filo non è per nulla facile da individuare e seguire in quella selva interminabile di cinquecentotrentaquattro sonetti. 
Più facile, semmai, riconoscervi nuclei tematici, col conforto anche delle due didascalie poste alle pagine 179 e 2Is, di «Sonetti pastorali» a Annibal Caro $e$ «Pastorali. A Giovanvettorio Soderini» (la terza, a p. 229, riguarda una circostanza puramente esteriore: «Alcuni sonetti del medesimo autore, parte ritrovati nello stampare e parte aggiunti di nuovo»), e ancor più da appunti presenti nella ricchissima eredità di carte del Varchi conservate nelle Filze Rinnuccini della Biblioteca Nazionale Centrale di Firenze, nella filza I4, inserto 78 , cc. $357 v$ e 358 r. Precede a c. $357 r$ a mo' di titolo: "Ordine della prima parte de' sonetti / Sonetti del Varchi. I». Segue sul verso quest'elenco incolonnato a sinistra (le sbarre indicano a capo; il resto - uso di maiuscole e minuscole, segni d’interpunzione, lineette - è riprodotto fedelmente): «Pastorali ultimi / Primi - secondi - e terzi / Ducali / Carini primi - e secondi / Hercolani / Farnesi / Mendozzi / Curradini / Infermi / Morti / Artefici nobili / Prelati / Soldati / Monti / fiumi e fonti / Ville / Donne nobili: d'Amore / letterati / Bembi / Giovani di virtù / Lelii / Oradini / Vivaldi / Leone d'Arezzo / Temporali idest d'anni / Ad Asinarum / De Asinaro / Ad Laurum et de Lauro / Di fantasia . et stravaganti / Ad Amicoss; verso destra, separati dai precedenti da una linea ondulata: «Di Bologna / fuorusciti» (all'altezza di «Mendozzi》 e «Curradini»). A c. 358 r, altro elenco in gran parte coincidente nei lemmi in piccola nel loro ordine: "Ad Laurum De Lauro / Ad Asinarum - De Asinaro / Ad montes / Ad fontes fluvios / Di fantasia . stravaganti / Donne. Amori-Nobili-Laudi / Prelati / Sodales Ad Amicos familiares. Di Bologna, Fuorusciti / Ad Invenes De virtute / Ad litteratos Bembo / Ad Artifices nobiles. Leon d'Arezzo / Ad Duces et milites / Infermi / Morti / Temporali idest d'anni / Ducali / Farnesii / Mendozzi / Curradini / Ad Carinum De Carino primi et 2 ${ }^{i}$ / Ad Herculanum De herculano / Ad Laurum De Lauro / Pastorali primi $-2^{i}$. terzì. E certo che gli elenchi non si riferiscono direttamente alla stampa del 'ss, nella quale, come si vedrà, più d'una categoria non trova riscontro, altre ve lo trovano dubbio o ancipite, tanto meno l'ordine di nessuna delle due versioni; è probabile che si riferiscano a un progetto di ristampa che l'autore non poté realizzare. Difatti in fine di quell'inserto $78, c .375 v$, si legge, scritto a foglio capovolto: «Sonetti prima parte - ristampanda».

Ma la gran parte dei nuclei elencati si riconoscono anche nella stampa del 'ss e possono essere un utile traccia per non perdersi nel bosco. Dopo i due sonetti proemiali, i numeri III-XX rispondono senz'altro alla categoria ad Laurum, de Lauro, inteso come emblema e senhal, esaltando e invocando tutti l' «arboscel》, le «fronde», l'«arbusto», l'«arbor sacro del sol》, la «pianta gentil», l"«alloro». Vi si può riconoscere, anticipata proprio al primo posto e ingrandita da uno a diciotto la funzione di Rvf. s: esaltazione del nome. Segue la serie XXI-XXIX, ad Asinarum, de Asinaro, sulla cui pendice, a Bivigliano, 
nominato a XXV 6, sul finire d'agosto (il 28?) del I527 all'ora sesta, come appunto preciserà il XXXVI, il Varchi conobbe Lorenzo Lenzi (Lauro). ${ }^{7}$ E questa per dire e celebrare il luogo dell'innamoramento. Dal quale con naturalezza la descrizione s'allarga ai circostanti dintorni fiorentini sulla destra dell'Arno: gli altri monti $o$ ad montes, XXX-XXXV, $e$ fiumi e fonti $o$ ad fontes fluvios, XXXVI-XLIV, per lo più benedetti da un'occasionale presenza del Lauro, magari in compagnia dell'autore. I seguenti, XLV-L, sono ancora su questi luoghi, ma si riferiscono a uno o più episodi: il ritorno al monte dell'incontro amoroso, cioè monte Senario in una suggestiva luce lunare (XLV), e a Fiesole (XLVI-L), alcuni (XLVI, XLVIII, XLIX) alludendo all'assedio di Firenze e deprecandone gli effetti su quel paesaggio. ${ }^{8}$ Vi s'aggiunge il LI, su due colline alla sinistra dell'Arno, Rusciano e Giramonte, in grazia della rievocazione anche in questo (5-I4) dell'assedio. Per LII-LXII di nuovo soccorre con esattezza un lemma di quegli appunti delle Filze Rinuccini: temporali idest d'anni, cioè anniversari dell'innamoramento, fra il quarto e il venticinquesimo in ordine progressivo. Sonetti d'anniversario, di questo o d'altri eventi ce ne sono stati e saranno ancora, a parte l'ultimo, disseminati nel libro: il XII $e$ XIII, per esempio, che stanno nella sequenza ad Laurum et de Lauro $e$ sarebbero potuti stare benissimo in questa. Ma questa è e si volle che fosse la serie compatta dei sonetti temporali. Anche il successivo, LXIII, lo è, ma l'anniversario è il nono da quando «la Brenta e 'l gran Bembo lasciai» (3). Non sarà li per caso, ma per affinità, quasi a sfumare il variare d'argomento particolare; che in generale, compreso questo e fino al LXIX rimane l'amore del Lauro, fuor che il solo LXIV, e piu il ricordo del tempo e luogo dell'innamoramento, nell'ultimo toccando anche d'un anniversario, il ventiquattresimo.

La prima svolta decisa viene ora, dopo i primi sessantanove sonetti, dedicati, come s'è visto, all'amore del Lauro secondo le categorie petrarchesche, ben dilatate, del nome, del luogo e del tempo. Dal LXX alla fine ci saranno anche sonetti sul Lauro o al Lauro o, senza senhal, a Lorenzo Lenzi, spesso e volentieri sul luogo o $i$ circostanti luoghi dell'innamoramento, che s'impongono come protagonisti inscindibili dall'oggetto umano dell'amore e delle poesia, su vari episodi o circostanze (assenze, ritorni, visite, eventi della sua carriera ecclesiastica), ma saranno isolati o in coppie o in serie per lo più brevi: per esempio, LXXXVII e LXXXVIII, XCI, C e CI, CLXVI, CCXLIX e CCL, CCLIXCCLXI, CCLXIII, CCLXVII-CCLXX, CCLXXV-CCLXXVIII, CCCIV, CCCVII e CССVII, СССХ-СССХII, СССLXXVI (chiamato Dafni, trattandosi di sonetto pastorale), CCCLXXXII, CCCLXXXVI, CCCXIV-CCCXVI, CCCCLXXXIX, collocati, forse, di preferenza in punti nei quali, come si vedrà, non si ravvisa alcun motivo o solo un blando elemento che riunisca nuclei definibili. Altre volte del Lauro è più o meno fuggevolmente richiamato l'amore o la presenza in sonetti d'altro principale argomento, per esempio nel XC (nel successivo emergendo in 
primo piano), CLXVIII e CLXIX, CLXXIV-CLXXVI, CLXXXII, CCXXXIV, CCLIII, CCLVII, CCLXXXIX, CCXCVIII, CCCLXXXIV, CCCXVII-CCCXIX, CCCXLIII e CCCXLIV, CCCXLVII. Né fra questi due elenchi d'esempi è sempre facile distinguere; ma importa e basta che in tutta l'interminabile raccolta l'amore del Lauro e, ripeto, dei luoghi dell'amore sia una presenza effettiva e unificante del libro. Il quale, però, dopo i primi sessantanove sonetti si scandisce secondo nuclei d'altro argomento. I numeri LXX-LXXXIV rispondono al lemma composito Donne nobili, d'amore, e nell'altra poco chiara versione Donne. Amori - Nobili - Laudi. Ma non c'è dubbio che i sonetti da LXX a LXXXII siano d'amore, non per il Lauro, ma amore passione, e a LXXIV-LXXVII, LXXIX, LXXX e LXXXII certamente per donna; LXXXIII e LXXXIV sono in lode di due nobildonne: Beatrice Pia degli Obizzi e Camilla Strozzi de' Malvezzi (dove secondo l'uso cinquecentesco il secondo cognome preceduto da preposizione degli, de' è quello del marito). Segue l'LXXXV, alla Gloria formalmente assimilabile ai precedenti per la sua raffigurazione in persona di donna e l'invocazione iniziale: "Donna, ch'bor di sudor piena e di polve». Altra personificazione a LXXXVI, il Sonno, e percio legato all'ultimo, ma in nessun modo assimilabile al gruppo. Con questo si è sotto ogni aspetto fuori dal nucleo sulle donne e gli amori e fino al CI non è facile individuare motivi o altri elementi che riuniscano $i$ testi, se non per XCIV-XCVII le lodi di Lodovico e Ugolino Martelli, Vittoria Colonna, ignoto toscano defunto (le Laudi che nell'appunto di Filze Rinuccini I4, c. 358r, sono nel lemma poco chiaro Donne. Amori - Nobili - Laudi?). Invece, anche se la categoria non compare in quegli appunti, CII-CVII, sono sonetti per lo più spirituali (il CV, che si rivolge al papa, potrà classificarsi come religioso); cui ne seguono altri, CVIII-CXX, che esprimono un'interiore sofferenza, tutti chiusi da una preghiera a Dio, CXXI. Succedono e ben si riconoscono le sequenze degli Infermi, CXXII-CXXVII, e dei Morti, CXXXVIII-CLXV, cioè sonetti per malattie e di compianto funebre.

Da qui, CLXVI, a CCCI, una lunga e centrale porzione del libro, si possono riconoscere nuclei ben chiari e anche definiti dagli appunti della filza Rinuccini; ci si riferisce a nuclei in sé compatti, ma che difficilmente o forse mai riuniscono tutti $i$ sonetti che per argomento o destinazione vi potrebbero essere inseriti: letterati $o$ ad litteratos, CCII-CCXXII, entro $i$ quali si segnala per ampiezza la serie al Bembo, $i$ Bembi CCX-CCXVI; artefici nobili o ad artifices nobiles, CCXXXIX-CCXLIII; Lelii (cioè a Lelio Bonsi), CCXLVIIICCLVII, e Oradini (cioè per Lucio Oradini), CCLVIII-CCLXII;'9 soldati $o$ ad duces et milites, CCLXXXI-CCXC. Si potrebbe isolare una serie ad amicos $o$ ad amicos familiares, CLXXIII-CLXXVIII; qui, più nutrita che in appendice agli amori, una a donne nobili, CLXXXIX-CXCIII. Per $i$ prelati ce ne potrebbero essere tre: CLXX-CLXXII, CCXXIV-CCXXVI, CCLXVII-CCLXXIII, ma 
nessuna o per la scarsa quantità o per intrinseca o relativa debolezza convince; per $i$ fuorusciti o in ogni modo sulla perdita della libertà di Firenze e conseguente diaspora i CCXXVII-CCXXXI; ma perché il sonetto a Iacopo Nardi ne è separato e di cosi poco, сСXXXVI? Fuori dai lemmi degli appunti della filza Rinuccini si ravvisa un nucleo piccolissimo ma definito e calibrato: CLXXIX, a Michelangelo, CLXXX a e sull'amico di lui Tommaso Cavalieri, CLXXXI, all'amico proprio Lorenzo Lenzi sull'Aurora e la Notte di Michelangelo. Si potrebbe ravvisare un gruppo di sonetti di commiato e lontananza nei numeri CXCIV-CCI, anche se n'andrebbe levato il CXCV. Ma questa categoria, come anche altre a partire dal CLXVI e fino al CCCI, è indotta dalla vera costante di quest'ampia porzione del libro, che di regola, tolti, se non sbaglio, CLXIX, CCXV, CCLXIX, CCLXXVIII, CCXCV, è fatta di sonetti di corrispondenza. L'impostazione, certo, è ricorrente anche in altre parti del libro. E non mi riferisco all'invio, e verrebbe da dire dedica, che nella tavola alfabetica degli incipit ne accompagna la più gran parte, ma alla formulazione epistolare del testo con apostrofe a una persona reale e viva, costituita dal nome o da un titolo o dal semplice pronome e verbo di seconda persona, come in diciotto, per esempio, dei trentotto sonetti funebri, $i$ morti, CXXVIII-CLXV, in uno dei sei infermi, CXXII-CXXVII. Fra $i$ ventuno sonetti, CII-CXXI, che resultano o spirituali o di sofferenza interiore, ma per i quali non sovviene un titolo dalle carte dell'autore, il numero di quelli epistolari si rialza a undici, concentrandosi nei CXI-CXIX, preceduti dal CV (al papa). In precedenza, oltre LXXXIII, LXXXIV, XCII, XCV, non ne trovo (non si tien conto dei sonetti in lode o d'amore svolti in seconda persona o rivolti a entità personificate). Il fenomeno comincia, dunque, col nucleo CII-CXXI, precisamente col CXI. Ma solo nel tratto in questione, CLXVI-CCCI, ampio e centrale, si può parlare piuttosto di costante che di tendenza epistolare. Nei successivi undici, CCCII-CCCXII, cinque sono di corrispondenza, che era anche in precedenza, CI-CLXV, più o meno la media. Né altro li unifica tutti, $i$ singoli come le sequenze ben individuate; che di per sé rimangono tali, ma, senza saldarsi l'un l'altra, per cosi dire, vi galleggiano, come, nel magma, separati coaguli.

I CCCXIII-CCCXX sono $i$ Farnesi, cioè per il cardinale Alessandro Farnese e personaggi del suo seguito in occasione d'una sua visita a Fiesole (CCCXIII), ospite di Cosimo (CCCXIV). Succedono i Mendozzi, CCCXXI-CCCXXX, per il cardinale Francesco Mendoza de Burgos e il seguito in visita a Fiesole (ancora questo luogo) e ai santuari toscani (Vallombrosa, Camaldoli, La Verna, Certomondo). Ecco, infine $i$ ducali, CCCXXXI-CCCLI, per tutta la famiglia regnante, compreso il cameriere Sforza Almeni, che apre la sequenza, come in funzione di portinaio, undici a Cosimo, due alla madre, Maria Salviati (uno in morte), due alla duchessa, uno ciascuno a cinque figli maschi, Francesco, Giovanni, Garzia, Ferdinando, Antonio. 
La lunga e ben visibile serie encomiastica potrebbe segnare già di suo un forte stacco, rilevato anche tipograficamente da quel che segue, un titolo su pagina bianca (179), Sonetti Pastorali, con una generale dedica a Annibal Caro, costituita dal primo sonetto, CCCLII, p. I80a. Quelli a tema e ambientazione propriamente pastorale arrivano al CCCLXXVI; da CCCLV a CCCLXIV si canta l'amore di Filli e Damone (nome pastorale del Varchi); da CCCLXVIII a CCCLXXIII quello di Licori e Iola, ma anche di Damone per Iola. Succedono con funzione di cesura due sonetti epitalami, CССLXXVII e CССLXXVIII; quindi sonetti, almeno in massima parte, per o su Giulio della Stufa, in alcuni designato col nome pastorale di Carino, CCCLXXIX-CCCCXX. Gli appunti della filza Rinuccini parlano di tre serie di pastorali e di due di Carini. Ecco, difatti, un altro stacco grafico a p. 2I4, occupata da un sonetto solo, CCCCXX, invece che dai soliti due, e a p. 2Is, di nuovo il titolo Pastorali con generale dedica «a messer Giovanvettorio Soderini» e un solo testo. Questa serie, CCCCXXI-CCCCXLVII ha come protagonisti Carino, Nape e Damone e un luogo definito: la valle dell'Ema e Vacciano, sulla sinistra dell'Arno, oltre il colle d'Arcetri. È una regione simmetrica per due rispetti, posto l'asse dell'Arno e la conformazione valliva, al monte Senario, Fiesole e gli altri poggi alla destra del fiume, come nel libro questi ventisette sonetti finali sono speculari ai XXI-L che in principio descrivono quei luoghi dell'incontro e dell'amore col Lauro. Formalmente, difatti, la raccolta finisce qui col commiato da una poesia posta tutta alla sua ombra: "Queste cosè cantò mentre ch'all'ombra / Sedea Damon, di quel sacrato alloro / Che l'Arno e 'l Tebro co' suoi rami adombra; / Hor di cura maggior la mente ingombra / E posto mano a più grave lavoro, / Il canto e'l suon dal cor per sempre sgombra) (CCCCXLVII, 9-I4), cui segue sul piano ideale e progettuale la chiusa col computo cronologico e il positivo bilancio del DXXXIV.

Nelle carte del libro a stampa si frappongono a mo' di dichiarata e non ordinata appendice gli ottantasette sonetti "parte ritrovati nello stampare e parte aggiunti di nuovo» (p. 229): CCCCXLVIII-DXXXIV. Anche questi hanno in massima parte carattere epistolare, qualcuno pastorale. Oltre al consueto ricordo del Lauro è parecchio presente l'amore per Giulio della Stufa o Carino $e$ il motivo del suo traviamento. Si possono riconoscere alcuni nuclei, come quello funebre di CCCCLVI-CCCCLXI e soprattutto quello, DXV-DXXII, celebrativo d'un bronzo di Leone d'A rezzo raffigurante Filippo II: gli appunti della filza Rinuccini I4, cc. $357 v$ e 358r, registrano Leone d'Arezzo. La serie è inserita in un contesto milanese e lombardo, nel quale lo scultore operava, facendo appello ai letterati locali, preceduta da un sonetto, DXIII, a Francesco Vinta, ambasciatore mediceo a Milano, e da un altro, DXIV, a Girolamo Cardano e seguita da uno, DXXIII, a Giuliano Goselini. ${ }^{\text {IO }}$ Idealmente $i$ componimenti di quest'appendice si dovranno distribuire ai luoghi loro nelle rico- 
nosciute sequenze all'interno del libro, che, come detto, si chiude con l'addio alla poesia del cCCCXLVII e col bilancio di quello effettivamente ultimo, DXXXIV.

Dunque la prima parte dei sonetti (t5 5), costituisce il canzoniere di Benedetto Varchi, aperto e chiuso nel nome del Lauro, nella prima sezione (fino a LXIX) dominato $e$ in seguito variamente segnato e percorso dal motivo di quell'amore $e$ del luogo di quell'amore. Tuttavia il libro anche si articola rispecchiando in maniera assai diretta le relazioni sociali e culturali, insomma la vita pubblica dell'autore, se non nelle due serie dei sonetti pastorali, più squisitamente letterarie o, meglio, impostate secondo questa tipica convenzione e finzione letteraria; le quali sole, difatti, sono contrassegnate e isolate da un particolare titolo. Di conseguenza al di fuori di queste ultime e della prima sezione prevale il tono epistolare, che nel cuore magmatico del libro è parso unico elemento unificante e che sarà unica ragione di $\mathrm{t57}$. A questo s'accorda e assimila un'altra peculiarità della raccolta varchiana, quella d'esser fatta solo di sonetti: il genere metrico deputato allo scambio o, tecnicamente, tenzone, quindi il genere metrico dialogico, epistolare e sociale per eccellenza. Allora a progettare e costruire un altro libro, strettamente coordinato nel titolo come seconda parte, in cui anche comparissero le risposte e le proposte dei corrispondenti, forse l'autore fu indotto sia dal numero spropositato di sonetti che dovevano essere sistemati (si sarà facilmente intuito che alla selezione fosse poco portato) sia e più sostanzialmente dall'impossibilità oltre un certo limite di far convivere la forma canzoniere, su cui con ogni evidenza vuol modellare t5 5, col debordante carattere epistolare di tanti sonetti e lunghe serie di sonetti, che, seppure rime di corrispondenza sono raccolte in ogni canzoniere, in questo caso minacciavano di sformarlo e snaturarlo. Ė come se quello del Varchi, o prima parte dei sonetti, cosi largamente impregnata di questa dimensione epistolare e di relazioni sociali, se ne dovesse sgravare, almeno un po', partorendo un secondo libro, la seconda parte ( 5 7), che schiettamente e pienamente fosse un epistolario in versi, un frutto che per la conformazione, una voce costante di riferimento, sempre alternata a quella dei risponditori o dei proponenti, trova pochi o punti esempi o analogie.

Non è, questa del parto, una metafora vaga o al massimo un'ipotesi non verificata né verificabile per la genesi dei due libri diversi ma coordinati in una prima ( 55 ) e una seconda parte dei sonetti (t57). Ci sono, difatti, due codici della Biblioteca Nazionale di Firenze, il Magl. VII I073 (M) e il II VIII I43 $(\mathrm{N})$, che portano questo titolo: I sonetti di m. Benedetto Varchi, con alcune proposte, et risposte [risposte, e proposte $\mathrm{N}$ ] di diversi, in $\mathrm{N}$ corretto su: I sonetti di $\mathrm{m}$. Benedetto Varchi insieme con alcune risposte e proposte da diverse persone sopra varie materie mandategli, e che contengono riuniti in un unico libro e sotto quel titolo unitario con 
scarse diversità reciproche nelle presenze un gran numero dei sonetti delle due parti che appariranno separate nelle stampe del 'ss e del's7, progressivamente avvicinandosi all'ordinamento dell'una e dell'altra.

Prima di descrivere e esaminare $i$ due codici in rapporto alle stampe bisogna ricordare che delle carte di Benedetto Varchi è pervenuta una ricchissima e assai compatta eredità, riunita nelle Filze Rinuccini della Biblioteca Nazionale di Firenze e nei codici di provenienza Rinuccini distribuiti nel fondo principale della medesima biblioteca, come il codice $\mathrm{N}$, ma anche altro non poco di diversa provenienza alla Nazionale, come il codice $\mathrm{M}$, e nelle altre biblioteche fiorentine, senza escludere affatto quelle non fiorentine, sicché $i$ dati che saranno qui esposti sono parziali anche rispetto a quanto è disponibile. In particolare le Filze Rinuccini, benché esplorate solo in parte, di sicuro conservano altro che interessa l'argomento. A parte raccolte di sonetti anche parecchio consistenti, come la filza 3, cc. 7 I sgg., in cui si riconosce l'ossatura di 55 e t57, ma che sembrerebbero collocarsi in un periodo posteriore, forse in vista d'una progettata ristampa, non anteriore e in preparazione, se ne trova almeno una ragguardevole che a un primo esame appare precedente. È la filza I4, inserto numerato 77, cioè cc. 268-319, fatto di tredici quaderni con numerazione originaria a pagine, I-I04, e un sonetto per pagina. Tenuto conto dei resultati che si esporranno di seguito, questa raccolta molto parziale rispetto alle stampe del 's $e$ del 's7 $e$ dei due codici che si esamineranno, sembra collocarsi in una fase anteriore a quelle come a questi, ma forse per certe connessioni ben riconoscibili incamminata sulla stessa strada. Sia essa frammentaria o appartenga a un diverso più breve disegno, s'è preferito accantonarla, restringendo l'esame alle due più ampie di $\mathrm{N}$ e di $\mathrm{M}$, nel contenuto delle quali si riconoscono per intero differenti fasi del progetto consegnato alle stampe $\mathrm{t} 55$ e $\mathrm{t} 57$. Si considerano, dunque, queste con la coscienza che altre testimonianze manoscritte sulla genesi delle due stampe esistono e che il quadro potrà essere integrato anche di molto.

Il II VIII I43, della Biblioteca Nazionale Centrale di Firenze (N), ${ }^{\text {I }}$ di provenienza Rinuccini, è un codice cartaceo del XVI secolo, di cm. $27 \times 17$, di 192 carte numerate originariamente e esattamente a pagine, cominciando dalla terza, pp. I e 2, fino alla centottantacinquesima, $p$. 365 e 366, le seguenti, come la prima, sono bianche, salvo un'annotazione di mano cinquecentesca sul verso dell'ultima: "carte [?] I69», come anche la p. 366, a parte la nota che registra l'entrata in Magliabechiana del codice dalla biblioteca Rinuccini in data "V Id. Mart. [II marzo] I850». Sono dodici fascicoli d'otto fogli ciascuno, non numerati e senza richiamo; raccolti già nella biblioteca dell'autore in una legatura membranacea a busta, che porta scritto all'esterno del piatto anteriore in grafia cinquecentesca: $" G$ sonetti con risposte». La lettera $G$, difatti, sembra trovare corrispondenza nell'Inventario de' libri del Varchi 
(Filže Rinuccini II, inserto 49, cc. 265-344), c 335r, ossia la sezione di Rime del Varchi in penna in $4^{\circ}$ per l'A.B.C., dove sotto $G$ si registra: $« I^{\circ}$ libro in carta pecora in $4^{\circ}$ ' (in cartapecora s'intenderà la legatura). C'è una mano fondamentale seppure con variazioni anche accentuate, che scrive il testo dei sonetti, due per pagina, corretti, oltre che dalla stessa, da una scrittura più calligrafica e rigida, d'impostazione e con caratteristiche più arcaiche, che mette anche gran parte dei nomi degli autori ai sonetti di risposta o di proposta, o da una corsiva ben riconoscibile per quella dell'autore, che intervengono anche reciprocamente l'una su correzione dell'altra. Quindi anche le scritture di per sé non riconducibili all'autore sono da lui guidate e controllate

Sul recto della seconda carta c'è il sonetto con attribuzione a «M. Michelagnolo Vivaldi» Voi che leggete in varii suoni eletti, che invita a esaltare lo stile d'un innominato, dalle evidenti caratteristiche del Varchi poeta, scritto dalla mano fondamentale, anche se più allentata (viene a occupare due terzi della pagina) e la seconda terzina, scancellata, è riscritta, variata, dalla scrittura più calligrafica delle correzioni; in fine $\mathrm{M}$. Mi, sbarrato con due freghi (s'era ricominciato a scrivere il nome dell'autore?). Sul verso il titolo riferito sopra in capitali sistemato al centro della pagina, sicché il sonetto I della prima parte a stampa, cioè di $\mathrm{t5} 5$, Quel ch'Amor mi dettò, viene a occupare la parte inferiore libera, riducendo il margine. Sembra che in origine la pagina prevedesse solo il titolo e che questo sonetto proemiale vi venisse aggiunto in un secondo tempo. La pagina I e quindi il corpus originario comincia col sonetto III e prosegue nelle successive con due sonetti per pagina, fuor che la 317 che n'ba uno. Sulla 204 fu incollato con gocce di ceralacca (ora s'è staccato) un foglio delle stesse dimensioni su cui fu ripetuto il numero e furono ricopiati, inserendo a testo le correzioni, $i$ due sonetti (proposta e risposta della par. $2^{a}$ CXXI). Il nome dell'autore della proposta, Mattio Franzesi, che era, come per solito, della scrittura calligrafica d'alcune correzioni, nella seconda copia è della mano dei testi. Lo stesso avviene a p. IgI solo per la metà inferiore (risposta a Bernardino Tomitano, par. $2^{a}$ CLVIII). Tracce di ceralacca anche su p. I88; ma non trovo la carta che vi potrebbe esser stata sovrapposta. Da $p .3 I 7$ alla fine la scrittura si fa nettamente più trasandata, dilatandosi verso il margine inferiore e quelli laterali. Sono le pagine degli ultimi due fascicoli, scalettate sul margine esterno e, fino a p. 353, contrassegnate da una lettera dell'alfabeto (da A a $\mathrm{V}$, senza $\mathrm{K}$ ), come le nostre rubriche, forse predisposti per un indice alfabetico e poi usati per altri sonetti. Un mutamento analogo, benché più contenuto, di scrittura e anche d'inchiostro s'avverte alle pp. I68-72 e 274-79, meno p. 278 . I sonetti contenuti in queste pagine non sono contrassegnati da un numero, che, come si vedrà, deve riferirsi a un loro riordinamento, eccetto l'unico di p. 317, e $i$ due, ai quali fu depennato, di p. 278. A p. I29 si nota un altro cambiamento nell' inchiostro e anche un certo ricompattarsi della scrittura 
e $i$ sonetti delle pp. I24a-29b non hanno il numero, meno quello di p. I28a. Da qui, cioè da p. I29, a p. I68 c'è il primo blocco di corrispondenza con proposta dell'autore e risposta (prima ce ne sono solo tre, alle pp. I00-IO e I24); il secondo blocco col Varchi in veste di risponditore occupa le pp. 173-273. Altro sonetto suo con risposta, senza numero, a p. 276, uno di Lucantonio Ridolfi, Parmi, Varchi, ogni dì più di mille anni, con due risposte alle pp. 280-8Ia L'arbor gentil che forte amai molti anni $e$ Nell'altrui dolci rime $i$ tristi (su vostri) affanni; altri scambi di sonetti alle pp. 282 (senza numero di riordino), 3II, 3IS (senza numero di riordino) e non pochi da p. 318 in poi, tutti senza numero. Fra $i$ due blocchi maggiori e le pagine che precedono e seguono e nell'intervallo che li separa, come s'è visto, si osservano variazioni di scrittura e d'inchiostro. Il sonetto di p. I28b (CCLXVII) e quello di p. 28Ib, Hor che due volte cinque lustri intorno, portano l'annotazione autografa «ultimo», questo spicca anche per la scrittura di modulo più piccolo e più serrata. Altra decisa variazione d'inchiostro distingue quello di $p .292 b$ (CCLXVIII), che non ba numero, come il precedente (CCXXI), a p. 292a. In queste pagine segnate da variazione d'inchiostro e di grafia e dall'assenza del numero di riordinamento $i$ nomi dei corrispondenti sono sempre della mano dei testi, invece che della corsiva d'impostazione più calligrafica e rigida, cui spettano tutti gli altri, compreso il nome di Lucantonio Ridolfi a p. 280 e, con qualche dubbio, di Lelio Bonsi a p. 3II; inoltre i sonetti non seguiti da risposta, che nelle altre e anche in gran parte di queste non sono preceduti dal nome di colui al quale si rivolgono, alle pp. $274 a$ (valido anche per quello di 274b, al medesimo Giovanbattista Tedaldi), 277a, 279b, 283a, 288a, 288b, 292a, 292b, 294b, 333a, 333b, 345a, 348a, lo portano scritto dalla stessa mano del testo. Se la medesima differenza distingue la prima pagina 204 dalla seconda incollata sopra, è probabile che anche in queste altre tradisca un momento successivo della copia. La stessa differenza e la stessa dedurione s'estendono anche a alcune pagine contigue in cui non si apprezza con sufficiente chiarezza variazione d'inchiostro e scrittura e i numeri di riordinamento per solito accompagnano i sonetti, cioè alle pp. I67, 273, 286a (completato dalla corsiva del Varchi), 317 coinvolgendo potenzialmente quelle intermedie. S'avverta, tuttavia, che a p. 295a "Alla Signora Donna Giovanna d'Aragona» per CXCIII è supplito dalla corsiva del Varchi. Insomma, al di sotto della superficie, cioè la situazione finale del codice, piuttosto caotica, sembra d'intravedere uno strato anteriore di sonetti, che, dopo il titolo di c. $I V$, cominciava di sicuro a p. I col III, in cui probabilmente erano state lasciate alcune pagine o mezze pagine bianche, che furono riempite in seguito, come gli ultimi due fascicoli, destinati, parrebbe, a un incipitario. Se la struttura della silloge che costituisce lo strato originario, togliendo il sonetto I, quelli delle pp. I24-28 e I68 (anzi probabilmente già I67)-72, appare abbastanza definibile fino a $p$. 
272 e, dopo altre pagine bianche, pp. 280a-8Ib, benché si debba ritenere aperta, per l'ipotesi, ora formulata, di queste pagine in origine bianche, in seguito sfuma e il codice da copia a pulito, pur fortemente rivista, dopo aggiunte plurime e stratificate assomiglia alla fine e in certi punti, almeno gli ultimi due fascicoli, fin da subito a un deposito di materiale da ordinare. E possibile che esaminando la composizione di $\mathrm{N}$ nella più ampia dinamica redazionale che coinvolge M, 55 e 557 si possa determinare in modo meno vago anche la distinzione fra quello ora definito primo strato e i successivi, o meglio, fra cio che presumibilmente appartiene a una trascrizione in ordine e il materiale da ordinare collocato in carte disponibili. Ma l'esame delle scritture o l'osservazione di altre costanti esterne non permettono d'essere più precisi.

Alcuni sonetti soprattutto di quello che pare individuarsi come primo strato furono espunti e talvolta riscritti più o meno variati altrove con o senza avvertenza o con avvertenza generica o incompleta, di regola in pagine successive, più spesso verso la fine, quindi in una parte del codice che non pare mai appartenuta a una trascrizione in ordine, ma adibita fin da principio a deposito. Per esempio, CXXXV, cassato a $p .32 b$, è riscritto senza avvertenza a p. $283 b$; LXXVI $\grave{e}$ a $p$. $46 b$ scancellato con l'avvertenza autografa: "scritto a 356», dove, infatti, si ritrova; nessuno dei due è mai accompagnato dal numero di riordino. LXXVII era a $p$. 49 a con numero di riordino, fu sbarrato e spostato secondo indicazione autografa a p. 356b. CLIII, espunto a $p .42 b$ con l'indicazione autografa «a carte», si ritrova a $p .313$ col numero di riordino I23. Solo il XLIX della parte seconda, che è scritto con la risposta a p. 282, si ritrova da solo e scancellato a $p$. 294b, mai accompagnato dal numero di riordino, senza varianti che permettano di stabilire quale delle due copie preceda. Sopra s'è rilevato qualche indizio che nessuna delle due pagine appartenga al primo strato. C'è anche il caso d'un sonetto che compare due volte senza essere espunto mai: il LXXVIII della parte seconda si trova col numero di riordino $e$ con la risposta a p. I67, senza l'uno e l'altra a p. 2886. Ma stavolta ci sono varianti che fanno ritenere questa copia anteriore a quella. A p. 288 ci sono le seguenti correzioni autografe (in tondo le parole scancellate; non si ripete ciò che resta invariato): 4 "Si stan qual bronzo, in ben fondata basa» $\rightarrow$ «O marmo in ferma salda»; 7 "Di virtù, scaltro ingegno, e mente intera» $\rightarrow$ «senno et eloquenza intera»; I4 "Gli altri son foschi e torbidi splendori $\rangle$ «falsi》. A p. I67 le correzioni sono tutte inserite a testo, con una sola piccola incertezza: a 4 《salda» è sostituito a margine con «ferma», la prima soluzione, superata inter scribendum, del margine di p. 288. Pare, allora, confermato che la $p$. I67 era stata lasciata bianca e vi fu inserito in ordine un sonetto, con la risposta, che attendeva in pagine adibite a deposito. Altri furono spostati in punti del codice in cui, invece, non c'era posto per ritrascriverli interamente o con indicazione precisa del punto, per esempio il 
XCV da p. I9 a p. I6a dopo il XCIV (nel senso, quindi, di t5 5), dove il Varchi annotò: "qui s'ha a scrivere o di candido e terso a Ig»; o con indicazione della sezione: CXLV a p. 48a, non depennato, ha l'avvertenza: «ne' morti»; CXXII è cassato a p. 65a e ha l'avvertenza della scrittura più calligrafica d'alcune correzioni: «fra le malattie»; al CLXVI, p. 89a, non espunto, fu annotato: «tra quei di morte». In questi casi $i$ numeri essendo segnati ora accanto alla trascrizione espunta o comunque superata (LXXVII) ora accanto alla seconda (par. $2^{a}$ LXXVIII), significherà di per sé che gli spostamenti avvennero ora dopo ora prima del riordino; quando non vi siano mai, significherà che lo spostamento avvenne prima e che il sonetto piuttosto che spostato fu accantonato $e$ rimase in sospeso finché il Varchi lavorò su N. Ci sono anche sonetti scancellati e non ritrascritti in questo codice e che nemmeno mi resultano mai recuperati, come, per esempio, a p. ob S'a sì rara beltà voglie e costumi. L'altro codice, (M) Magl. VII I073 (provenienza Strozzi, in 4, I33, già 237), è cartaceo del secolo XVI, di cm. 27,7×I6, di carte VIII, I87, numerate modernamente; numerazione originaria a pagine con inizio a c. I fino a 370 , che omette di numerare le due facciate delle cc. 95 e 98 e ne corregge e sostituisce altre interrotte prima della fine ( $i$ testi saranno di regola indicati con riferimento a questa, non alla cartulazione moderna). Si tratta di quattordici fascicoli. Il primo, ternione, è costituito dalle cc. II-VII; il secondo, setternio, da VIII a I3, cui è unita da una braghetta I; seguono altri cinque setterni, cc I4-83, un fascicolo d'otto fogli, cc. 84-99, quattro setterni, cc. 100-55, e due fascicoli d'otto fogli, cc. Is 6-87. Tutti i fascicoli hanno richiamo in fine fuor che il primo e gli ultimi tre. Al settimo, c. II3, un primo vARC[HI] fu rettificato in Bench[è]. Probabilmente fu una banale svista: col vocativo «VARCHI», messo in evidenza dalle capitali, comincia il secondo verso del sonetto di c. II4r (р. 223a), la proposta di Girolamo Mentovato, par. $2^{a}$ CLXXXVII. Il codice è scritto da una sola mano, la stessa del II VIII I43 (N), che qui esegue anche la gran parte delle correzioni, non di banali sviste di copia, ma di portata redazionale e avverte di sonetti da inserire in questo o quel punto; altre correzioni ai testi e disposizioni strutturali sono nella corsiva tipica del Varchi. La scrittura d'impostazione più calligrafica e rigida, che corregge l'altro codice, in questo sembra riconoscersi alle pp. 353-69, di certo nell'estesa correzione a p. 340, ma con minor sicurezza anche in altre qua e là più circoscritte. Anche qui la corsiva del Varchi e la mano base intervengono reciprocamente l'una su correzioni dell'altra almeno nel sonetto di p. 220b. Ma, oltre questa circostanza, l'ampiezza e la qualità delle correzioni conferma ancora in modo evidente che, se la scrittura base di questo come dell'altro codice non appartiene all'autore, che assumerebbe in modo forse troppo deciso e poco credibile caratteri più formalizzati, ̀̀ d'un segretario o collaboratore che scrive sotto dettatura o sotto il controllo stabile di lui. 
Nel codice si distinguono nettamente tre sezioni: la più estesa e centrale è quella delle cc. Ir-I Ir (pp. I-297), seconda nell'ordine, preceduta sul verso di c. VIII dal titolo in capitali, a eccezione d'alcune e, minuscole ingrandite, riferito sopra, non esattamente centrato, ma appena spostato verso il primo terzo della pagina. I sonetti riconducibili a questa sezione, due per facciata, cominciano con I a p. I. In fondo a p. 297, dopo il sonetto Hor che due volte cinque lustri intorno è scritto in capitali, con la solita e minuscola ingrandita, IL FINE. La p. I42 è lasciata bianca e alla successiva cominciano $i$ sonetti di corrispondenza con risposta o proposta. Propriamente la p. I42 intatta non è, portando scritto in cima: "la proposta di questo è all'ultima faccia»; nella quale un sonetto di proposta c'è, del Varchi a Michelangelo Vivaldi, par. $2^{a}$ XXXI. Difficile è capire a quale si riferisca questo che sarebbe la risposta; non certo a quello che precede a p. I4Ib, CCCLXXVI. Pur con questo piccolo mistero è indubitabile la funzione di quella pagina sostanzialmente e intenzionalmente bianca di segnare il passaggio fra la serie continua dei sonetti del Varchi, che vengono prima, e quelli di corrispondenza con proposta o risposta, che segue, quasi altrettanto compatta, con pochi scompagnati: sei tutt'insieme a p. 188 (c. 94v)-c. 95v, altri quattro a c. 98r-v. Quello di Lucantonio Ridolfi, Parmi, Varchi, ogni dì più di mille anni, ha due risposte, L'arbor gentil che forte amai molti anni $e$ Nell'altrui dolci rime i tristi affanni ( $p$. 296-97a) come in $\mathrm{N}$, e, come in $\mathrm{N}$, è seguito da Hor che due volte $e$ IL FINE. La scrittura di questa sezione, come in genere anche quella delle altre due, è regolare e uniforme; solo quella del sonetto LXXXVI a p. I27b è più serrata e di modulo più piccolo; meno accentuata, ma apprezzabile la riduzione del modulo a p. I88 e alla seguente c. 95; la scrittura di c. 98 appare meno accurata, specie nel tenere l'allineamento delle righe, e si dilata verso $i$ margini superiore e inferiore.

La sezione seguente, terza nell'ordine, cc. ISIV-I87v (pp. 298-370), ancora con due sonetti per facciata, fuor che l'ultima, che n'ba uno, scritto largamente e cominciato a circa un terzo della pagina, ha sul margine superiore della prima l'avvertenza: "Questi sottoscritti sonetti s'hanno a scrivere tra gli altri a" luoghi loro come si vedrà di mano in mano». E ciò di suo designa la sezione centrale come trascrizione in ordine. Nella quale, difatti, vi sono inseriti tutti col richiamo dell'incipit e indicazione della pagina in cui si trovano fino a $p$. 330, con l'eccezione del CCLXI, $p$. 3I9b; in seguito sempre meno, finché da $p$. $342 b$ nessuno è recuperato, fuor che la risposta al XXXVI della par. $2^{a}, p .353 a$. S'indicherà come $\mathrm{M}^{\mathrm{I}}$ la sezione centrale $e$ in ordine aggiornata con questi inserimenti. Invece ci sono diversi sonetti li cassati e riscritti qui, a partire da p. 342, ora punto ora più o meno mutati. Si dà anche il caso d'uno, la proposta della par. $2^{a}$ LXIII, scancellato a p. II3a, riscritto con la risposta a p. 342, quindi reinserito in ordine a $p$. I60, facendo un viaggio d'andata e ritorno. 
Questa sezione delle pagine finali appare rispetto a quella centrale e in ordine come una specie di retrobottega o deposito con materiale in attesa d'essere messo, diciamo cosi, in mostra e in cui ne viene appoggiato altro che per il momento n'è tolto. Anche in $\mathrm{N}$ s'è rilevato lo stesso fenomeno, di sonetti scancellati e riscritti più avanti, verso la fine, dove il codice non ha più l'aspetto di copia nemmeno in origine a pulito, ma di deposito di materiale da ordinare. La differenza è che qui, in M, si distingue bene e nettamente il passaggio fra quella che è venuto di chiamare mostra e quello che s'è chiamato e si seguiterà a chiamare deposito; mentre li, in $\mathrm{N}$, al posto d'un chiaro confine e tangibile, come una parete divisoria, s'avverte un passaggio graduale e uno stato di maggior movimento. Li la mostra e il deposito oltre che entità ravvisabili nello spazio, cioè nelle carte del codice, sono situazioni che si succedono nel tempo: quella che in origine era mostra, cioè trascrizione in ordine, affiancata da un deposito, gradatamente specie nell'area prossima al confine si confuse con questo, tanto che s'è parlato di strati.

Ritornando a M, resta da dire della prima sezione, priva della numerazione originaria a pagine, le cc. I-VIII, contenenti sonetti del Varchi di regola due per facciata, diversi con risposta o proposta, solo la c. I n'ba uno sul recto e un altro sul verso, scritti abbastanza largamente da non lasciare spazio a un secondo. Senz'altro proseguendo questa trascrizione a carattere provvisorio, come quella delle pp. 298-370, fu impiegata la parte inferiore di c. VIII, quella destinata al titolo. Lo mostra con chiarezza la scrittura, meno formalizzata e controllata di quella di $p$. I, che gli sta a fronte, e delle successive, con gli "in fuori" in principio di quartina e terzina, che non isolano, come li, la sola iniziale maiuscola, ma vi sono subito attaccate le lettere seguenti. Questa sezione, dunque, si configura come un secondo, piu piccolo deposito di sonetti non ancora in ordine; nessuno dei quali resulta richiamato e inserito nella sezione centrale e in ordine.

Già da questa descrizione si percepisce $\mathrm{M}$ come posteriore a $\mathrm{N}$ o almeno più definito e in quella sezione centrale delle pp. I-297, chiusa da IL FINE, più assestato, seppure soggetta a espunzioni e inserimenti $\left(\mathrm{M}^{\mathrm{I}}\right)$. Certo è che l'elaborazione dei singoli sonetti disegna in modo chiarissimo e senza equivoci la direzione da $\mathrm{N}$ a $\mathrm{M}$ a $\mathrm{5} 55, \mathrm{t57}$, assumendo per solito $\mathrm{M}$ l'ultima elaborazione di $\mathrm{N}$ e $\mathrm{t} 55$ o 557 l'ultima di M. Si dà qualche esempio indicando con $\mathrm{N}$ e $\mathrm{M}$ il testo base dei due codici e con $\mathrm{N}^{\mathrm{a}}, \mathrm{N}^{\mathrm{b}}, \mathrm{M}^{\mathrm{a}}, \mathrm{M}^{\mathrm{b}}$ le successive varianti (l'interpunzione e l'uso delle maiuscole sono posti secondo l'uso moderno). XXV (N p. 7b; M p. ob; 555 p. I5) 5-8: «Da indi in qua come si pianga e canti, / S'arda insieme e s'agghiacce, viva e mora / Provato ho sempre, e come in men d'un'hora / Mille varietà soffran gli amantì N M, "In te bel monte, che di te t'ammanti / E col tuo Biviglian vagheggi Flora, / Mirai quel tronco giovinetto allhora, / Di cui sempre convien ch'io pense o 
canti》 $\mathrm{M}^{\mathrm{a}}$ t5 5; II «crebbe la pianta» N M, «Scors'io la p. » $\mathrm{M}^{\mathrm{a}}$ t5 5; I2-I3 "Qui lieti un di, s'al ver presago sono, / Spargeran fiori e fronde i pastor toschi» N, "Qui lieti un di, s'alme presaghe sono, / Spargeran latte e fiori $i$ pastor toschi》 $\mathrm{N}^{\mathrm{a}} \mathrm{M}$ t5 5. XLVII (N p. I7a; M p. I3a; t5 5 p. 26b) 5-8: «In te lungi dal mondo e dagl'inganni / Farò, sicuro omai, dolce soggiorno, / Ridendo hor lungo un rivo hor sotto un'orno / Il folle vaneggiar de' miei primi anni» N, "In te lungi dal mondo e da suo inganni / Farò, sicuro omai, dolce soggiorno, / Pensando hor lungo un rivo hor sotto un'orno / Quanto chi'l ciel non segue invan s'affanni» $\mathrm{N}^{\mathrm{a}}$, "In te lungi dal mondo e da suo [suoi M] inganni / Farò sicuro omai dolce soggiorno / Pensando hor lungo un rivo hor sotto un'orno / Quanto chi segue non il ciel s'inganni» $\mathrm{N}^{\mathrm{b}} \mathrm{M}$ t5 5. CIV ( $\mathrm{N} p$. 28a; M p. 35a; t5 5 p. 54b) 5-8: «E se ben Signor mio varcato bo 'l segno / Amando eguale a te cose mortali, / Perdonami Signor, che con queste ali / Volo anche spesso al tuo stellante regno» $\mathrm{N}$, «E se forse tal'hor varcato ho'l segno, / Amando oltra il dever cose mortali, / Ben sai tu, Signor mio, che con quell'ali / Sole men volo al tuo stellato regno» $\mathrm{N}^{\mathrm{a}}$, «E se forse tal'hor varcato ho 'l segno, / Amando oltra il dever cose mortali, / Ben sai tu, Signor mio, che sol queste ali / Ho da volare al tuo stellato regno» $\mathrm{N}^{\mathrm{b}} \mathrm{M}$, «E se pare ad alcun ch'io varche il segno, / Amando, come te, Fronde mortali, / Ben sai tu che sol queste e non altre ali / $\mathrm{Ho}$ da volare al tuo stellato regno» $\mathrm{M}^{\mathrm{a}} \mathrm{t} 55$. CLXXIV (N p. 66a; M p. 74a; t5 5 p. 89b) 5-8: «Non v'incresca per me, ch'alto pensiero / Mi tira e dolce natural disio, / Dove spero apparar, non pur disio, / La cagion delle cose e'l sommo vero» $\mathrm{N}$, «Non v'incresca per me, ch'alto pensiero / Mi spigne e» $\mathrm{N}^{\mathrm{a}}$, «Non v'incresca per me, ch'alto pensiero / Mi sprona, e sferza natural disio / Spregiar quel ch'altri cerca e gir dove io / Appari oprare il buon, sapere il vero» $\mathrm{N}^{\mathrm{b}} \mathrm{M}$, «Non v'incresca .... / Appari oprare il ben» $\mathrm{M}^{\mathrm{a}} \mathrm{t} 5$ 5. Gli esempi potrebbero moltiplicarsi, e di molto; ma basteranno questi a segnare con certezza la direzione delle varianti e a scandire la cronologia relativa delle testimonianze redazionali, che naturalmente tollera eventuali recuperi. S'aggiunga solo questo, in cui la correzione riguarda il computo d'un anniversario e si configura proprio come aggiornamento cronologico: ХХХІІІ (N p. 7a; M p. 9a; t5 5 p. I6b) II: "Già s'avvicina il quindicesimo anno» $\mathrm{N} \mathrm{M}$, "Già s'avvicina il sestodecimo anno» $\mathrm{M}^{\mathrm{a}} \mathrm{t} 55$. S'è detto che in $\mathrm{N}$ quasi tutti $i$ sonetti sono accompagnati da un numero, che solo qualche volta è progressivo. Ė facile constatare che essi corrispondono all'ordinamento di $\mathrm{M}$ con pochi e piccoli aggiustamenti (soprattutto sonetti che o non si trovano in $\mathrm{N}$ o vi si trovano senza numero e evidentemente furono inseriti nel libro organizzato soltanto con $\mathrm{M}$ ) e con scarsi errori: qualche numero saltato o ripetuto; in due casi la ripetizione è di un'intera decina, 340-349 e 360-369; più singolare il caso d'una decina saltata, da 220 a 230, non in sé, ma perché in $\mathrm{M}$ i nove sonetti corrispondenti ci sono, alle pp. IIob-I4b 
(sono CCXCIX, CCXCIII, proposta di par. $2^{a}$ LV, CCXLVI, CCXLVII, proposte di par. $2^{a}$ LXIII e par. $2^{a}$ XLVII, CCXCV, CCLXVII), tutti provenienti da pagine di $\mathrm{N}$ non coperte dalla trascrizione in ordine. E come se quel buco fosse stato lasciato in $\mathrm{N}^{\mathrm{I}}$ col proposito di riempirlo; oppure il riordinamento di $\mathrm{N}^{\mathrm{I}}$ e la conseguente trascrizione in ordine in $\mathrm{M}$ furono contemporanei o quasi e per quei dieci sonetti fu saltato il primo passaggio? In ogni modo è sicuro che l'ordinamento di M nasce, nella sostanza, come trascrizione a pulito dei mutamenti e della maturazione intervenuti in $\mathrm{N}$ sia nella lezione sia nella struttura $\left(\mathrm{N}^{\mathrm{I}}\right)$. Ben chiaro e indubbio, dunque, in che modo le testimonianze e le fasi redazionali che se ne possono ricostruire si succedano: $\mathrm{N}, \mathrm{N}^{\mathrm{I}}, \mathrm{M}, \mathrm{M}^{\mathrm{I}}$, $\mathrm{t} 55 / \mathrm{t} 57$.

Tralasciando in un corpus tanto esteso l'evolversi del dettato, se non quando serva a capire come si succedano le copie d'uno stesso sonetto, avvertito solo qui $e$ in generale che non pochi a carattere epistolare, ma senza risposta, mutano destinatario, si può vedere un po' più da vicino, scorrendo $i$ codici e soffermandosi in qualche punto a titolo d'esempio, come si determini a partire da $\mathrm{N}$ l'ordinamento, che approda alle due parti separate di $\mathrm{t5} 5$ e $\mathrm{t57}$, con le rispettive scansioni, nella prima su base tematica, illustrate in principio e che ora bisognerà tenere costantemente presenti e talvolta richiamare a rischio di qualche ripetizione. Già in $\mathrm{N}$ il Varchi pensava secondo categorie del genere, viste le annotazioni ricordate sopra, descrivendo il codice, per spostare CXLV da p. 48 «ne' morti» e di CXXII da p. 65 «fra le malattie». Il dato è portante, perché autorizza a leggere la storia dell'ordinamento fin da $\mathrm{N}$ secondo quelle categorie, che, semmai, a quell'altezza potevano non essere predominanti, ma che di certo esistevano. Né è difficile ravvisarvele, anche se con assai minore nettezza, oltre che differenza nella composizione dei nuclei. Netto fin da $\mathrm{N}$ (pp. Ia-4b), a dare la direzione e l'impressione del canzoniere per il Lauro, l'avvio nel suo nome, ad Laurum, de Lauro, III, IV, VI-VIII, X, XI, LXIII, richiamando subito anche la peculiarità, se non lo scandalo di quell'amore ("Arsi a me saggio altrui folle ed ardito / [...] / Quindi ogni altra mia speme, ogni disio / Di santo e bello oprar bebben radice; / Che dica o pensi il volgo audace e rio», III 7, I2-I4, N p. I), accennando ben presto anche allo snodarsi d'una vicenda: un allontanamento $(X)$, un anniversario indeterminato (XI). $V i$ s'aggiungeva il LXIII, toccando d'un altro episodio, la dimora padovana, anzi il nono anniversario della sua fine, per far ritorno alla «pianta del sol» (4), e nel contempo replicandone le lodi ("O sacre foglie ...» I2-I4), ragione della sequenza, che cosi pertanto si chiudeva. Questa, compreso il LXIII, resterà stabile fino a $\mathrm{M}\left(p p\right.$. 2b-6a), inserendosi solo il $v$ in $\mathrm{N}^{\mathrm{I}}$ col numero 5 , li dove rimarrà per sempre, e in coda $\left(\mathrm{N}^{\mathrm{I}}\right.$ I2 e $\mathrm{M}$ p. 6b) il XVI, che in $\mathrm{N}$ era poco sotto in queste pagine iniziali (p. Isa). $\mathrm{M}^{\mathrm{I}} v^{\prime}$ aggiunge il $I X\left(p .4 b^{I}\right)$, XIII e XII, in quest'ordine $\left(p . s b^{I}, 5 b^{2}\right)$, e il XV (p. $\left.6 a^{I}\right)$; anche altri (XVII, XVIII, XX), ma 
in altre pagine seppure iniziali, confermando che queste costituiscono una sezione di base tutta sull'amore del Lauro, scandita in sottoinsiemi. Fra quello ad Asinarum (pp. 5a-8b) XXI-XXIV, XXVIII, XXV-XXVII e il successivo ad montes ( $\not p$. IOa-IIa) XXXII, XXXIV, XXXV, strettamente collegati secondo un procedimento estensivo, $\mathrm{N}$ aveva due sonetti né all'uno né all'altro omogenei: il XIX, che alla fine, in $\mathrm{t} 5 \mathrm{5}$, finirà con buona ragione in quello iniziale, ad Laurum, de Lauro, e uno che comincia S'a sì rara beltà voglie e costumi, subito soppresso. Similmente fra la terna ad montes e quella ad fluvios fontes, XLIII, XLIV, XC (pp. I2a-Iza) s'inseriva l'LXXXIX ( ritorno della primavera e dell'amore. Né sarà facile a XIX e LXXXIX trovare un posto plausibile. In $\mathrm{N}^{\mathrm{I}} i$ due sonetti, numerati 4 I e 42, e $\mathrm{M}$ (p. 2Ia-b) s'annideranno nel successivo interstizio, dopo fiumi e fonti e prima dei temporali idest d'anni. Il secondo, anzi, non lo troverà mai; in M prima fu cassato, quindi recuperato, annotandovi l'autore: «scrivasi》, $e$ in $\mathrm{t} 5 \mathrm{f}$ fini a $p$. $47 a^{12}$ in compagnia d'altri non riducibili sotto generale etichetta, cioè LXXXV (p. $45 a)-C I(p ., I b)$.

S'è parlato per $\mathrm{N}$ (pp. I2a-Iza) di terna Ad fluvios, formata da XLIII e XLIV, enumerazione di corsi d'acqua del bacino dell'Arno: «Sieve, Era, Elsa, Mugnon, Bisenzio et Arno», ripetuta a mo' d'anadiplosi e cerniera nell'ultimo e nel primo verso dei due sonetti. Il terzo, XC, porta, invece, lontano, cominciando: "Adice e Po, che 'l fral di me portate». Questo in $\mathrm{N}^{\mathrm{I}}$, col numero 39, e in $\mathrm{M}($. 20a) rimarrà unito al gruppo aperto dagli altri due e nel frattempo accresciuto con XXXVII, XLI, XLII, XXXVIII e XXXIX, numeri 34-38 di $\mathrm{N}^{\mathrm{I}}$ e pp. I7b-19b di $\mathrm{M}$, su altrettanti torrentelli o rigagnoli della destra dell' Arno (quelli che identifico), dal Terzolle alla Mensola; ma in 55 finirà in quella medesima serie varia delle pp. 45a-sIb. La sorte è condivisa dal XCI, rivolto agli enganei Altero Venda e Ruvolon, che $\mathrm{N}^{\mathrm{I}}$ aveva recuperato dal deposito delle carte finali di $\mathrm{N}$ (p. 29Ia) dandogli il numero $3 I$ in coda ai sonetti sui poggi che contornano Firenze sulla destra dell'Arno, XXXII, XXXIV, XXXV di N, 28-30 in $\mathrm{N}^{\mathrm{I}}$; e $\mathrm{M}$ (pp. I 4 b-I6a) aveva confermato. Ma è chiaro che XC e XCI non potevano stare in quella che oltre il pretesto o $i$ pretesti legati al Lauro e all'amore per lui (che di per sé giustificava la presenza di questi due e dell'LXXXIX) si configura come una sorta di guida di quei dintorni fiorentini.

A questi sempre più o meno si legano dei sonetti incentrati sul tema del ritorno al luogo dell'innamoramento, cioè monte Senario, in un caso, a Fiesole, negli altri, che nei piu si determina dopo l'assedio del I529-30 e si fa contemplazione dei suoi effetti su quel paesaggio. Si tratta nella sistemazione finale di $\mathrm{t} 5 \mathrm{~s}$ dei $X L V-L$, dietro la sequenza fiumi e fonti, XXXVI-XLIV, a chiudere cosi l'intera sezione dei luoghi. Il nucleo, poco mutato l'ordine (XLVI, XLIX, XLVIII, XLVII, L) e assente XLV (inserito in $\mathrm{M}^{\mathrm{I}}$ a p. Io dopo XXVII, tratto dal deposito 
di $\mathrm{M}, p .337 a$, e di $\mathrm{N}$, p. 35Ia), era in $\mathrm{N}^{\mathrm{I}}$ (23-27) e $\mathrm{M}$ (pp. I2a-I4a) fra le sequenze ad Asinarum, XXI-XXIV, XXVIII, XXV-XXVII $\left(\mathrm{N}^{\mathrm{I}}\right.$ 13-20, $\mathrm{M} p p$. 7a-Iob) $e$ ad montes, XXXII, XXXIV, XXXV $\left(\mathrm{N}^{\mathrm{I}} 28-30, \mathrm{M}\right.$ pp. I $\left.4 b-I 5 b\right)$ : già, dunque, saldamente inserito nei luoghi, anzi nella parte di questi più omogenea, quella dei poggi. Anche $i$ due che qui immediatamente seguono ad Asinarum, XXXI e XCII ( $\mathrm{N}^{\mathrm{I}} 2 I-22, \mathrm{M}$ p. II $\left.a-b\right)$ facevano parte del gruppo. Il primo, XXXI, tolto dal deposito di $\mathrm{N}(p .3$ IIOa) è precisamente un soggiorno di «dodici e dodici hore» (I3) a Fiesole e apre benissimo l'argomento; può resultare, semmai, sacrificato nella più anonima collocazione di $\mathrm{t} 5 \mathrm{5}$, secondo su Fiesole e della serie ad montes (XXX-XXXV). Il secondo, XCII, è un'allocuzione ai «forti guerrier'» al tempo dell' assedio acquartierati sul «bel poggio» che nel 406 aveva visto la sconfitta di Radagaiso, cioè Fiesole, e aveva buone ragioni d'essere subito prima dei sonetti che gli effetti dell'assedio contemplano sul paesaggio fiesolano. Purtroppo, vien da dire, t5s lo sentì estraneo e lo relegò in quella specie di ripostiglio interno occupato da LXXXV-CI. Anche in $\mathrm{N}$ questo gruppo c'era, aperto proprio dal XCII e composto inoltre da XLVII, XLVI, XLIX, XLVIII (PD. I6b-I8b); non ancora, però, cosi strettamente legato alla sequenza sui luoghi, essendone separato da quella dei, per allora solo tre, sonetti d'anniversario, temporali idest d'anni LIII, LVIII, LXI (pp. I I b-I uno sul Lauro XVI (p. Isa), due lodi di poeti XCVI ( $p$. Isb) per Vittoria Colonna, XCIV (p. I6a) per Ludovico Martelli. Lo seguivano (p. I9a-b) due per Ugolino Martelli, XCV e Se da grandine e pioggia, cassato. Dopo questi e assai lontani dai loro tre compagni di pp. I2a-I3a (XLIII, XLIV, XC), $o$, per meglio dire, da quelli che diventeranno in $\mathrm{N}^{\mathrm{I}}, \mathrm{M}, \mathrm{t} 5 \mathrm{~s}$ loro compagni, s'incontrano due sonetti su corsi d'acqua, il Terzolle e il Rimaggio (o un Rimaggio, data la frequenza di questidronimo intorno a Firenze e in Toscana), XXXVII e XLI (p. 20a-b); ai quali presto s'aggiungeva XLII con un'avvertenza sul margine inferiore della p. 20 di $\mathrm{N}$, diciamo $\mathrm{N}^{\mathrm{a}}$ : "Qui s'ba scrivere il sonetto Vezzoso fonte .3I6.», tratto appunto dal deposito di p. 3166. In queste prime venti pagine di $\mathrm{N}$, in definitiva, si ritrovano i germi di tutte quelle che saranno le sequenze iniziali di $\mathrm{t} 55$; ma, se non si tenesse conto di questo punto d'arrivo, difficilmente potrebbero ravisarsi, per essere mescolate fra loro e a sonetti d'altro soggetto. Il momento in cui questi germi vengono isolati e prendono a svilupparsi come $i$ segmenti in cui s'articola la prima sezione della raccolta è $\mathrm{N}^{\mathrm{T}}$. Ciò avviene creando necessariamente quella specie di cassa di compensazione che in $\mathrm{t} 55$ è formata da $L X X X V-C I$ e in $\mathrm{N}^{\mathrm{I}}-\mathrm{M}$ era ancora un piccolo nucleo: $\mathrm{N}^{\mathrm{I}}$ 63-66, $\mathrm{M}$ pp. 32a-33b (XCIV-XCVI, CLXIX). $E$ cosi anche nelle pagine successive di $\mathrm{N}$ sequenze tematiche omogenee possono trovarsi divise e intersecate da altre. Cosi quella di donne nobili: d'amore vede un primo troncone alle pp. 2Ia-24a, costituito da LXXII, LXX, LXXI, LXXVIII, Furia crudel, Deh, dimmi, Amor, Altera e vaga, e un secondo 
alle $p$ p. 45a-52b, costituito da LXXIII-LXXVI, LXXXIII, CCCCXCII, CXLV, Donna ch'a singular, LXXVII, CXCIII, CXC, LXXIX, LXXX, LXXXI, XCIII, LXXXII. Fra $i$ due s'interpongono sonetti o più facilmente coppie di sonetti, CLXIX, XCVII, XCVIII, CCLXII, CCLXIII, CII-CIV, CCCLXXVII, CCCLXXVIII (pp. 24b-29a), d'argomento, come si capisce anche dalla definitiva numerazione, disparato; e soprattutto la serie compatta di rime funebri, CXXVIII-CXXXIII, CXXXV, CXXXIV, CXXXVI, CXXXVII, CLXII, CXXXVIII, CXLIII, CXXXIX-CXLII, CLII, CXLIV, CXLVI, CXLVII, CCCXLIV,CXLVIII, CXLIX, CLIV, CLI, CLIII, CLVI, CL, CLVII, CLV (pp. 29b-44b). Tuttavia anche quelle d'amore erano, pur divise, due sequenze ben individuate e omogenee; eppure già in $\mathrm{N}$ sottoposte a parziale dispersione e revisione, che strettamente si collega e si congiunge a $\mathrm{N}^{\mathrm{I}}$ $e$ M. Furono scancellati da $\mathrm{N}$ e per sempre Deh, dimmi, Amor e Altera e vaga (che era per Piero Alberti) nella prima ( $p$. 23b, 24a), Donna ch'a singular nella seconda (p. 48b). LXXVI fu depennato da p. 46b, annotatovi "scritto a 356", dove verosimilmente fu trascritto a quel momento in una lezione che non muterà piü; ${ }^{3}$ il sonetto, difatti, rimarrà accantonato anche in $\mathrm{M}($. $348 b)$ per essere reintegrato proprio nel nucleo donne nobili: d'amore da $\mathrm{t} 55$. A CXLV (p. 48a) fu annotato «ne' morti» e qui lo colloca subito $\mathrm{N}^{\mathrm{I}}$ col numero II4, M (p. 58a) e infine 55 . CXCIII fu cassato da $p .49 b$ con rinvio generico «a carte» e si ritrova depositato a p. 295a, dove fu recuperato da $\mathrm{N}^{\mathrm{I}}$ col numero Is2, trascritto in $\mathrm{M}$ a $p .77 a$, pervenendo in $\mathrm{t} 5 \mathrm{~s}$ in quella sezione centrale che s'è definita genericamente di corrispondenza e in una sottosezione, CLXXXIX-CXCIII, di corrispondenza con donne, già delineata in $\mathrm{N}^{\mathrm{I}}$ e M. Anche CXC riceve in $\mathrm{N}^{\mathrm{I}}$ il numero I53, andando in $\mathrm{M}$ a $p .77$ b e rimanendo in 55 a far compagnia a CXCIII. LXXVII e XCIII furono cassati alle pp. 49a e sa con l'avvertenza, rispettivamente, «scritto a 356» $e$ 《a carta $360 »$, e alle pp. $356 a$ e $360 \mathrm{~b}$ si trovano in lezione assai diversa. ${ }^{\mathrm{I}}$ Gli altri, più questi ultimi, riordinati in $\mathrm{N}^{\mathrm{I}}$ come 47-62, secondo la successione LXXV, LXXIII, LXXIV, LXXII, LXX, LXXI, LXXVIII, Furia crudel, LXXIX, LXXX, LXXXI, XCIII, LXXXII, LXXVII, LXXXIII, CCCCXCII vanno a occupare le pp. 24a-3Ib di M. Due sono eliminati qui: Furia crudel, non recuperato, e CCCCXCII, ripescato nella sezione estrema delle novità e, appunto, dei recuperi di t5 5. Altri sono accantonati nel deposito: LXXII, corretto, è espunto a p. 25b, annotatovi: "Questo sonetto è scritto a 355 », e a p. 355 a è ricopiato con le correzioni; XCIII e LXXVII sono anche qui, come nell'altro codice, sbarrati con l'avvertenza, rispettivamente, "questo e riscritto a 3481$\rangle$ e «questo è riscritto a 34I», dove, pp. 348 a e $34 \mathrm{I}$ b, si ritrovano con le stesse varianti e minimi aggiustamenti. ${ }^{15} \dot{E}$ facile vedere che quanto resta, meno XCIII, ma compresi LXXII e LXXVII, formeranno in $\mathrm{t} 5 \mathrm{~s}$ la sequenza continua donne nobili: d'amore, riassunto anche LXXVI, di cui sopra, e inserito il solo LXXXIV. Si è cercato di seguire, cosi, pezzo per pezzo la storia d'un segmento della raccolta. E ciò valga, non 
potendo farlo per tutti, come caso esemplare. Ma, almeno questa volta, la vicenda fu ancor più travagliata e meno lineare di quanto fino a ora potrebbe apparire e, osservando certi movimenti interni alle singole e ben delineate fasi, si può arguire anche qualche generale deduzione. I numeri (si danno nell'ordine in cui si dispongono in $\mathrm{N}^{\mathrm{I}}$ e $\mathrm{M}$ ) LXXV, LXXIII, LXXIV, LXXVIII, LXXXI in $\mathrm{M}$ furono espunti e recuperati con l'annotazione "questo non ha essere scancellato» (LXXV, LXXIII, LXXIV) o "questo si scriva» (LXXVIII, LXXXI): normale ripensamento. Ma anche nel II VIII I43, cioè $\mathrm{N}$ prima del riordinamento, $\mathrm{N}^{\mathrm{I}}$ dopo, LXXV è cassato e in margine a LXXIII, LXXIV, pur non sbarrati, e LXXV (ordine di $\mathrm{N}$, pp. 45a-46a) si legge rispettivamente "questo si scriva», "re questo», "re questo». Il fenomeno è in tutto analogo a quello descritto sopra per XCIII e LXXVII. Che cosa vuol dire? Che, in un caso, $i$ sonetti furono due volte accantonati e recuperati, come, nell'altro, XCIII $e$ LXXVII furono depennati da $\mathrm{N}^{\mathrm{I}}$, che aveva assegnato loro il numero 58 e 60, quindi da $\mathrm{M}$ recuperati e di nuovo accantonati? Oppure, e piuttosto, il Varchi, anche dopo aver riordinato $i$ sonetti nel II VIII I43, $\mathrm{N}^{\mathrm{I}}$, e fatti trascrivere secondo il nuovo ordine in $\mathrm{M}$, non chiuse il primo codice, ma, saltuariamente, fece la stessa operazione sull'uno e sull'altro in parallelo? Simile movimento in parallelo si dà nelle pagine finali di deposito dei due codici: a p. $346 b$ di $\mathrm{N}$ e a p. $329 b$ di $\mathrm{M}$ si trova la proposta della par. $2^{a}$ XVI, cassata in tutt'e due e in tutt'e due riscritta identica con la risposta a $p .350$ di $\mathrm{N}$ e 334 di $\mathrm{M}$; ugualmente la proposta di par. $2^{a}$ XXIII si trova scancellata a p. $347 b$ di $\mathrm{N}$ e a p. 333 b di $\mathrm{M}$ e riscritta con la proposta a p. 353 di $\mathrm{N}$ e 338 di $\mathrm{M}$, intervenendo qui una correzione a IO-II che in parte (s'è detto: saltuariamente) è applicata su tutt'e due $i$ codici. ${ }^{16}$ Solo le pagine estreme di $\mathrm{M}$, da 36 I alla fine, le carte iniziali, I-VIII, in cui s'è ravvisato un secondo deposito, la p. I88 con la successiva c. 95 e la c. 98, che, come si capirà, erano state lasciate bianche, si possono ritenere scritte quando ormai il II VIII I43 era stato chiuso, visto che nulla del loro contenuto compare anche in questo codice.

Ripigliando a sfogliare $\mathrm{N}$ e soffermandosi sui nuclei definiti, si trova, preceduto alle $p$ p. $53 a-59 b$ da molti dei sonetti, o spirituali o improntati a interna sofferenza o nostalgia (CXV, CXIV, CVIII, CVI, CVII, CXIII, CIX, CX, CXV, CXIX, CXVII, CXVIII, par $2^{a}$ XLI, CXXI), che lo precederanno anche in seguito fino a 55 5, quello breve, ma già lì definito «malattie» (pp. 60a-62a): CXXIII-CXXVII. Questo perverrà intatto a $\mathrm{t} 55$, passando per $\mathrm{N}^{\mathrm{I}}$ e $\mathrm{M}(p p .46 a-48 a)$ aggiuntovi in testa ( $\not .45 b)$ il cXXII, che in $\mathrm{N}$ era poco lontano, a p. 65a. Tuttavia il suo viaggio fu piu lungo e non in tutto chiaro. Il sonetto fu sbarrato e spedito "fra le malatties; ma si ritrova a $p$. 289 a in una versione di molto variata e solo qui contrassegnata dal numero 88, primo, appunto, di questa sequenza, del riordinamento di $\mathrm{N}^{\mathrm{I}}$, quindi trascritto in $\mathrm{M} a p .45 b$. Qualcosa c'è da rilevare anche nella numerazione di $\mathrm{N}^{\mathrm{I}}$, nella quale, dopo 88, di cui s'è detto ora, si 
trovano 90, 93, 94, 95 a margine di CXXIV, CXXV, CXXVI, CXXVII; l'89 non c'è, $e$ andrà immaginato accanto a CXXIII, che non ha numero; ma nemmeno di $9 I$ e 92 trovo traccia e non posso pensare a altro che a un salto materiale. CCCXXXIX, per una malattia del duca, in $\mathrm{N}$ a p. 307a, fu inserito nel gruppo da $\mathrm{N}^{\mathrm{I}}$ col numero 94, assegnato anche al CXXVI, e da $\mathrm{M}$ sistemato in coda, p. 48 ; in 555 fu assunto fra $i$ ducali.

La differenza da $\mathrm{N}$ alle successive fasi è più forte nella combinazione dei segmenti. In $\mathrm{N}^{\mathrm{I}}, \mathrm{M}, \mathrm{t} 5 \mathrm{~s}$ la lunga serie dei morti succederà secondo l'ordine di natura alle malattie o infermi, mentre in $\mathrm{N}$, s'è visto, precedeva alle $p$. 29b-44b non solo le malattie, ma il secondo spezzone delle donne nobili: d'amore, mentre al suo interno era già ben definita. Sembra d'avvertire, semmai, una certa disponibilità di $\mathrm{N}$ a includervi sonetti che nemmeno apparissero in morte di qualcuno. Difatti il CLXVI posto a p. 89a, lontano da questa sequenza, descrive, in origine al Molza, sostituito già in $\mathrm{N}$ dall' «Arbor del sol», uno stato d'animo dolente in sintonia con una primavera piovosa; c'è anche un accenno non chiaro a «lei, che tutti ancide e null'ascolta», cioè la Morte, al massimo allusione indiretta e implicita a qualche recente perdita, per nulla vincolante (se mai c'era), visto il cambio di destinazione. Eppure vi fu annotato: «tra quei di morte». Ma non se ne fece di nulla; CLXVI rimase fino in $\mathrm{t} 5 \mathrm{~s}$ in quel lungo tratto vario e vago, in cui si trovava in $\mathrm{N}$ e la serie dei morti li e in seguito netta e concretamente in morte di definite persone. Nella parte ordinata di $\mathrm{N}$ e di $\mathrm{M}$ ancora mancavano rispetto a 55 CLVII e CLIX, che erano nel deposito di $\mathrm{M}($. $363 a-b)$; CLX e CLXIII, che erano sia in quello di $\mathrm{N}$ (pp. 362b e 363a) sia in quello di $\mathrm{M}$ (p. 359a-b); CLXIV e CLXV, che non erano nemmeno nei depositi (piangono la perdita recente, 23 aprile I554, di Gaspara Stampa). CLXI si trovava solo in $\mathrm{M}$ in pagine verosimilmente, come si vedrà, rimaste bianche fra quelle destinate alla corrispondenza con risposta o proposta, c. 98vb. Il CXLV per una signora Lucrezia da Pistoia già in $\mathrm{N} f u$ dirottato dal secondo troncone delle donne nobili: d'amore $(p .48 a)$ «ne' morti), dove $\mathrm{N}^{\mathrm{I}}$ gli diede il numero II4 e $\mathrm{M}$ la p. 58a. Tutti gli altri e in più CCCXLIV, in morte di Maria Salviati, che $\mathrm{t} 55$ sposterà fra $i$ ducali, erano fin da principio in $\mathrm{N}$ e passeranno tramite $\mathrm{N}^{\mathrm{I}}$, che presenta o dei banali incidenti o forse pentimenti di scarso rilievo, in $\mathrm{M}$ con qualche diversità nell'ordine fra loro e rispetto a $\mathrm{t} 5 \mathrm{~s}$ (quello di $\mathrm{N}$, pp. 29b-44b, s'è riportato sopra; ecco quello di M, pp. 49a-64a: CXXVIII-CXXXVIII, CXLIII, CXXXIX, CXL, CLXII, CXLI, CXLII, CXLIV, CXLV, CCCXLIV, CXLVI, CXLVII, CLII, CLIV, CXLVIII, CXLIX, CL, CLIII, CLVI, CLVII, CLV) e qualche ripensamento. Il CXXXV, in morte dell'Ariosto, s'interponeva in N (p. 32b) fra il penultimo e l'ultimo in morte di Giuliano Gondi, CXXXIII e CXXXIV; fu depennato e posto in aspettativa notevolmente variato a p. $283 \mathrm{~b}$, donde lo trasse direttamente $\mathrm{M}$ per porlo ( $p$. s2b) dopo l'ultimo sul Gondi. Il CLXII, a Veronica Gambara, chiedendole di 
consolare insieme al Varchi il Bembo per la morte della sua donna, veniva in $\mathrm{N}$ a p. $34 b$ dopo il сXXXVII; anche questo fu cassato e riscritto, ma in ordine, più avanti a p. I07b, in quella sezione centrale che, precorrendo l'assetto di t5 5 , ̀̀ apparsa dominata dal tono epistolare; fu recuperato alla serie mortuaria da $\mathrm{N}^{\mathrm{I}}$, che lo collocò col numero IIo fra il CXL e il CXLI, dove lo trascrive M (p. s6a). Il CLI in morte di Giovanni Mazzuoli detto Stradino e il CLIII in morte del Tribolo venivano l'uno dietro l'altro a $p .42$ di $\mathrm{N}$ dopo il CLIV in morte di Gismondo Martelli a Simone Strozzi, e furono tutt'e due espunti. Sul primo fu annotato: "Questo è scritto a carte I69", dove si trova con la sirima assai variata, in pagine che s'interpongono fra due blocchi di corrispondenza con risposte o proposte e che un po' si isolano per inchiostro e aspetto della scrittura. $\mathrm{N}^{\mathrm{I}}$ lo ignorò e $\mathrm{M}$ lo trascrisse nelle pagine finali di deposito ( $p$. 309a). $\mathrm{M}^{\mathrm{I}}$ lo riportò in ordine fra $i$ morti, annotandone l'incipit e scancellandolo, sia a p. 60 proprio dopo CLIV, che lo precedeva anche in $\mathrm{N}$, sia a p. 59 dopo CXLVII, in morte del Bembo; infine gli trovò pace a p. 58 dopo quello in morte, quindi all'ombra della sua protettrice Maria Salviati, CCCXLIV. Ma quando questo in 55 fu ricongiunto agli altri per la famiglia ducale, il povero vecchio e bizzarro soldato si trovo fra una signora, una Lucrezia da Pistoia, "Non men di quella antica o casta o bella» CXLV e CXVLVI un cardinale, e che cardinale, Gaspero Contarini, sicché dovette rimettersi in marcia, fino a trovar posto confacente in ragione del mestiere dietro a un gran capitano, anzi tre: Stefano Colonna, Giovan Battista Savelli, Pirro Colonna, CXLVIII-CL. Meno affannoso l'andirivieni del CLIII in morte del Tribolo: posto in attesa, variato, a p. 3Iз a di $\mathrm{N}$, qui lo recuperò subito alla serie $\mathrm{N}^{\mathrm{I}}$ col numero I23 e $\mathrm{M}$ lo trascrisse a $p$. 62 b per intanto fra il CL e il CLVI; $\mathrm{t} 55$ lo riporterà avanti a quel CLIV dietro il quale si trovava in $\mathrm{N}$.

Infine in questa sequenza dei morti si dà un caso curioso e forse istruttivo. $\mathrm{Nel}$ deposito di $\mathrm{N}$ a p. 316 a e 317 aspettavano due sonetti, il primo comincia Quanto dianzi alta, oimé, chiara e gentile, il secondo, intitolato «a mons. D'Arez:o», comincia Quanto stato per me fora il migliore e sarà il CCCCLXIV di t5 5 , cioè fra i sonetti "parte ritrovati nello stampare e parte aggiunti di nuovo». $\mathrm{N}^{\mathrm{I}}$ li segnò coi numeri, rispettivamente, I28 e I27. Ma M, invece d'inserirli semplicemente nella serie dei morti, come richiedevano quei numeri, all'ultimo e penultimo posto, li trascrisse per intero in una delle pagine finali (30Ia-b) nella stessa successione di $\mathrm{N}$. Tuttavia fra $i$ morti furono trascritti ( $p p .64 b$ e 65a) ancora in quest'ordine, non in quello richiesto da $\mathrm{N}^{\mathrm{I}}$, $i$ soli incipit, in piena pagina, non in margine, e lasciando in bianco lo spazio per il resto. Difatti gli incipit furono coperti con freghi di penna e sostituiti coi sonetti, interi, CCCXCII e CCCXCIII; che in $\mathrm{t} 55$ finiranno fra $i$ pastorali (riguardano Giulio della Stufa, Carino, rivolgendosi il primo ai suoi «terrestri soli / D'angioletto mortale», il secondo direttamente a lui) $e$ in $\mathrm{M}$ non 
chiudono, s'intende, la serie dei morti, che resta chiusa come in $\mathrm{N}$ dal CLV compianto di Pierino da Vinci, ma aprono la sezione centrale, varia e in prevalenza epistolare. La ragione di quegli incipit senza seguito è chiara, se si legge in che modo il primo Quanto dianzi alta, oimé continua in $\mathrm{N}(p$. 316a) o in $\mathrm{M}$ (p. 301a) e si capisce che piange la morte di Michelangelo: "Pianga l'Arte e rallegrisi Natura, / che quell'ANGEL divino all' alte ruote / Tornato nulla più del mondo cura» (I2-I3); e difatti troverà debito posto nelle Esequie del divino Michelagnolo Buonarroti [...], in Firenze, appresso i Giunti, I564, c. B[4] v. Del personaggio compianto nel secondo non so dir nulla, se non dedurre che nel I5s5 fosse effettivamente morto, ma da poco, se il sonetto per lui fu stampato in t5 5 nell'appendice di cui s'è detto. Certo, Michelangelo nel 'ss non era morto, tantomeno prima al tempo in cui si lavorava su $\mathrm{N}$ e M. I due sonetti, allora, erano nati da falsi annunci e furono a buon conto tenuti in serbo o erano proprio quelli che in gergo giornalistico si chiamano "coccodrilli"? Nei fatti lo furono. È un caso limite e palmare, ma forse non straordinario d'uso "giornalistico" del sonetto.

Tornando ai codici, s'è arrivati al lungo tratto centrale, da p. 62b a p. IIob di $\mathrm{N}$, in cui già trovavano posto di regola $i$ sonetti che costituivano il corrispettivo del tratto di $\mathrm{t} 55$ (e già di $\mathrm{N}^{\mathrm{I}}$, numeri I29-220, e M, pp. 64b-II4b), debitamente allungato, CLXVI-CCCI. Ci sono delle eccezioni a questa regola, ma sono proprio quelle che la confermano, o, meglio, confermano con somma chiarezza l'unica regola o denominatore comune individuato, considerando l'assetto definitivo di $\mathrm{t5} 5$, a questa sezione centrale, di riunire, cioè, sonetti epistolari. Difatti quasi tutti quelli che erano in $\mathrm{N}$ e che in seguito saranno levati sono sonetti di proposta che saranno ricongiunti alla risposta nella seconda parte: par. $2^{a}$ XXXV, par. $2^{a}$ XXXVI (pp. $\left.77 b-78 a\right)$, par. $2^{a}$ LI (p. 9Ib), par. $2^{a}$ XXXIV (p. 95b). Due sono di risposta già qui preceduti dalla proposta, d'Agostino Beaziano, par. $2^{a}$ CLXIX, e di Lodovico Dolce, par. $2^{a}$ CLXXIX (pp. I09-Io): sarebbero un caso estremo e strano; ma queste pagine sono la carta che precede la serie compatta dei sonetti pastorali dedicati al Caro e $i$ testi non hanno il numero di riordinamento di $\mathrm{N}^{\mathrm{I}}$; e perciò è possibile che la carta fosse stata lasciata bianca e venisse adoprata come spazio di deposito. Il CCCXXXI (p. I05b) a Sforza Almeni, cameriere del duca, che pertanto andrà, ma solo in t5 5, a aprire $i$ ducali è un sonetto epistolare alla seconda persona.

Di questa lunga sezione e dei suoi movimenti non si può render conto in modo capillare e completo. Oltre coppie e terne che resisteranno unite fino alla fine, in $\mathrm{N}$ vi s'isolano già definiti nel numero e nell'ordine $i \mathrm{Bembi}$ (CCX-CCXVI, pp. 85a-88a) che in t5 5 faranno da nocciolo a un gruppo di letterati $o$ ad litteratos (CCII-CCXXIII) e intorno ai quali già tendono a gravitare sonetti con questa destinazione: CCVIII e CCIX a Claudio Tolomei e Trifon Gabriele ( $p$. 84), CCIII e CCIV a Sperone Speroni e Girolamo Muzio (pp. 8rb-82a), CCVII 
alMolza (p. 88b), cCV е CСXVII a Luigi Alamannie Bernardo Capello (pp. 89b90a); più distanti, allepp.93 a e 106b, i CCI a Alberto Lollio e CCXVIII al Trissino. Ilprimo passo di $\mathrm{N}^{\mathrm{I}}$ consisterà nello sceverare in queste pagine ciò che eraperletterati da ciò che non era, determinandone la sequenza, comprensiva al suo interno dei $\mathrm{Bembi}$ (fra parentesi i numeri arabi del riordinamento, avvertendo che quelli mancanti mi resultano, salvo errore, saltati): CCIII (I64), CCIV (I65), CCVIII (I66), CCIX (I67)-CCXVI (I73), CCVII (I74), CCXVII (I78), CCV (I80), CСXVIII (I8I). M la trascrive alle pp. 83b-90b con l'inversione CСXVII (p. 89a)ССИII ( $\mathrm{N}^{\mathrm{I}}$ e ap. 94 bin $\mathrm{M} . \mathrm{t} 55$ non farà che riordinare ilgruppo, saldarvi in principio $C C I$ e aggiungervi CCII a Girolamo Ruscelli, un secondo sonetto, CCVI a Luigi Alamanni, e alla fine $i$ tre a Giovanni della Casa CCXIX-CCXXI, con una sorta d'appendice al nipote di lui, Annibale Rucellai, CCXXII, a cui segue il cCXXIII al segretario del Bembo, Cola Bruno. Quest'ultimo già gravitava vicino al gruppo dei letterati in $\mathrm{N}^{\mathrm{I}}$ (I60) e in $\mathrm{M}(p .81 a)$, mentre in $\mathrm{N}$ era ancora nelle carte di deposito (p. 286a). CCII e CCXIX in $\mathrm{N}$ erano insieme a p. 104a-b. Ilprimo, ignorato dal riordinamento di $\mathrm{N}^{\mathrm{I}}$, fu deposto nelle carte finali di $\mathrm{M}(p .308 a)$. Ma a p. 104, nello spazio fra $i$ due sonetti CCXCV e CCLXVII fu annotato rapidamente con penna grossa «Ferrino», cioè CCXCVII, per il medico Tommaso Ferrini, e «Ruscello», cioè CCII. Il secondo, numerato da $\mathrm{N}^{\mathrm{I}} 21$, , fu trascritto in $\mathrm{M}$ ap. Io7be $\mathrm{M}^{\mathrm{I}}$ v'aggiunse di seguito gli incipit di CCXX, CCXXI e CCXXII, traendoli dal deposito di $\mathrm{M} p$. 298a (Np. 127a), 298b (Np.292a),318a (Np.343a).

Precedeva in $\mathrm{N} i$ letterati, ben visibile, un drappello d'uomini d'arme (soldati; ad duces et milites), pp. 7Ib-74a: CCLXXXI al conte Claudio Rangone, CCLXXXII a Stefano Colonna, CCLXXXIII a Giordano Orsini, cCLXXXVIII a don Garzia di Toledo, CCLXXXIV a Carlo Orsini, CCLXXXVI a don Pietro di Toledo; che in $\mathrm{N}^{\mathrm{I}}$ e M, accresciuto di due (CСLXXXилI a Iacopo d'Appiano e CCLXXXIX a Gabriele Moles, già accoppiati in N, p. I03a-b), passerà dietro, tenendosi ancora abbastanza vicino ai letterati, ma diviso in due tronconi e con due infiltrati nel primo: $\mathrm{N}^{\mathrm{I}}$ I 87 (CCLXXXI), I88 (CCLXXX, primo infiltrato, a Giorgio Bartoli; ma parla dell'autore, non di lui), I89 (CCI, secondo infiltrato, a Alberto Lollio), I90 (CCLXXXII), I9I (CCLXXXIII), I92 (CCLXXXIV), $2 I I$ (CCLXXXVIII), $2 I 2$ (CCLXXXII), 213 (CCLXXXVII), 214 (CCLXXXIX); $\mathrm{M}$ (pp. 93b-96a, I05b-107a) in tutto uguale a $\mathrm{N}^{\mathrm{I}}$. In questo caso si deve dire di queste due fasi coincidenti ciò che altre volte s'è detto di $\mathrm{N}$, che senza questo e $\mathrm{t} 5 \mathrm{~s}$ sarebbe difficile e poco legittimo riunire la sbandata soldataglia. Ma il drappello si ricompatta in $\mathrm{t} 5 \mathrm{~s}$ e cresce d'altri due (CCLXXXV a Chiappino Vitelli dal deposito di N, p. 362a, e di M, p. 353b, CCXC a Mario Savorgnan, che s'aggirava nei paraggi degli altri sia in $\mathrm{N} p .82$, sia in $\mathrm{N}^{\mathrm{I}}$ 199, e in $\mathrm{M}$ p. 99b), presentandosi in ordinata formazione CCLXXXI-CCXC, ormai lontano dagli nomini di lettere. 
Si tralasciano più sparute compagnie, come, per esempio, alle pp. 96b-97b di $\mathrm{N}$ ( $\mathrm{N}^{\mathrm{I}}$ I82-84, M pp. 9Ia-92a) cCXXXIII, CCXXXIV, CCXXXV rivolti a Scipione Bianchini, presentato come un dotto amico del Bembo, a Fabrizio Garzoni, bolognese, presentato come studioso d'Aristotele e d'Ippocrate, al medico e botanico bolognese Luca Ghini, che arriverà compatta in $\mathrm{t5} 5$, aggiunto in testa da $\mathrm{M}^{\mathrm{I}}, p .90$, il cCXXXII, attingendo al deposito di $\mathrm{M}, p .302 b$ (e cfr. N p. 279b), per un altro bolognese, il filosofo aristotelico Ludovico Boccadiferro. Piuttosto si rileverà in $\mathrm{N}$ una corona meno trascurabile di cinque sonetti per Piero Alberti, che subito precedeva alle pp. 93b-95b: CLXXXV, a lui stesso, e, su di lui, Se da leggiadra impresa alto valore a Ventura (?) Strozzi, CCXLV e CCXLIV a Simone della Volta, par. $2^{a}$ XXXIV proposta a Filippo Angeni; che progressivamente s'impoverisce e disperde, rinunciando già in $\mathrm{N}$ a Se da leggiadra impresa, anticipando in $\mathrm{N}^{\mathrm{I}}$ CLXXXV al numero I35, p. 686 di M, riducendosi cosi agli ultimi tre elementi, 200-202 di $\mathrm{N}^{\mathrm{I}}, p p$. rooa-rora di $\mathrm{M}$, e alla solo coppia a Simone della Volta in $\mathrm{t} 5 \mathrm{5}$. Si rammenti che a p. 24 a era stato scancellato un altro sonetto per l'"Altera e vaga e pura e chiara Petra, / Che sopra l'Arno al destro lato splende», cioè lo stesso Piero Alberti. Si dà anche il caso di nucleo, piccolo, di $\mathrm{N}$, che si disfà, ma poi si riaggrega su un diverso equilibrio in $\mathrm{t} 55$. Descrivendo questo, s'è rammentata la terna CLXXIX-CLXXXI su Michelangelo, l'amico suo Tommaso Cavalieri e il terzo sonetto che, con bella mossa, Lenzi, voi dite il ver, se tali e tante, disegna una conversazione fra l'autore e il proprio amico appunto sull'Aurora e la Notte della Sagrestia Nuova. In N alle pp. 62b-63b la terna era, nell'ordine, CLXXXI, Più non mi par Bettin del dritto fore, ${ }^{17}$ sul medesimo tema, CLXXIX; CLXXX, lontano a p. 8ob, non era in causa. CLXXXI e Più non mi par furono depennati; a p. I28 fu riscritta la fronte del primo, seguita dalla sirima del secondo. Con le parti che avanzavano dell'uno e dell'altro se ne sarebbe potuto montare un secondo, ma qui non l'ho ritrovato, né in $\mathrm{M}$, né in $\mathrm{t} 5$ 5, sicché pare che di due ci si rassegnasse a farne uno. In $\mathrm{N}^{\mathrm{I}}$ e $\mathrm{M}$ CLXXIX, il nuovo CLXXXI, come CLXXX, sono dispersi coi numeri I6I, I8I e I47 $e$ alle pagine 8Ib, 92b, 74b. In 555 si ricostituisce la terna che s'è visto, spostando cosi il peso e l'argomento prevalente dalla celebrazione della Sagrestia Nuova a quella dell'amor platonico: sono le ragioni del canzoniere.

Degli altri più o meno definiti e visibili nuclei della sezione centrale di 55 quello dei CCXXVII-CCXXXI, che parlano dell'assedio del I530 e la conseguente diaspora dei repubblicani fiorentini non consisteva minimamente in $\mathrm{N}$ ( ССХХVII a p. 75b, ССXXVIII a p. I72b, ССXXIX a p. 92b, ССXXX a p. 64b, cassato per essere riscritto a $p$. 290b, e che prima era inviato a Baccio Tasio e prima ancora, pare, a qualcun altro che non riesco a leggere sotto la cancellatura di p. 64b, CCXXXI a p. 278b); ma già quagliava con almeno tre in $\mathrm{N}^{\mathrm{I}}$ I5) (CCXXVII), Is6 (CCXXIX), Is7 (CCXXX), e quattro in $\mathrm{M}$ pp. $786-80 a$ (a p. 80 a 
c'è l'ultimo, CCXXXI, che in $\mathrm{N}^{\mathrm{I}}$ aveva ricevuto il numero 278 , cassato e non sostituito da altro, ma nel riordinamento il 158 , che gli toccherebbe, non è assegnato a nessuno). In $\mathrm{M}^{\mathrm{1}}$ il nucleo era già completo, annotato l'incipit del ССХХVIII dopo il ССХХИII a p. $78 b$, con rinvio alle pagine finali, dove, $p .305 b$, è trascritto.

Nessuna traccia in $\mathrm{N}$ e nemmeno in $\mathrm{M}$ della serie di cinque sonetti CCXXXIXCCXLIII artefici nobile; ad artifices nobiles. Anzi solo il primo, al Bronzino, e il terzo, a monsignor Giovan Battista Ricasoli sul Perseo del Cellini erano trascritti in ordine (N pp. 79a, 105a; $\mathrm{N}^{1}$ 196, 218 ? cassato e sostituito con 220; M pp. 98a, IIoa). Il secondo a Alessandro Allori fu inserito, dal deposito di $\mathrm{M}$ p. 3086 (era anche in quello di $\mathrm{N}$ p. 333 ) in $\mathrm{M}^{\mathrm{I}}$, ma in altra compagnia, a $p$. II4a: segno che a quella squadra ancora non si pensava. Gli altri due attesero nei depositi di $\mathrm{N}$, pp. 36rb, $358 a$, e di $\mathrm{M}$, pp. $352 b$ e 35 I b.

Invece $i$ Lelii e gli Oradini, cioè $i$ sonetti per Lelio Bonsi e Lucio Oradini CCXLVIII-CCLX, benché ignorati oltre che da $\mathrm{N}$, anche da $\mathrm{N}^{\mathrm{I}} e \mathrm{M}$, aspettavano, sì, nei depositi, ma già vi si stavano schierando in formazione. Alle pp. 329a-32a di $\mathrm{N}$ e 3roa-Iza di $\mathrm{M}$ si trovavano sette Lelii nella medesima sequenza: CCXLVIII, CCLII, CCL, CCLI, CCLIII, CCLIV, CCLVII. In $\mathrm{N}$ questo è il massimo della loro aggregazione; mentre gli altri vagano nei paraggi da soli, cCLVIII a p. 328 a (ma precedevano alle pp. 326 e 327 proposta e risposta della par. $2^{a}$ CXLVI e CXLVII), CCLX a p. 342b, o in coppia, CCLIX e CCXLLX a $338 a-b, o$ in tre, alle $p$ p. $345 b-46 b$, CCLV, CCLVI e la proposta della par. $2^{a} X V I$ (cassata e ricopiata con la risposta a p. 350 ). Ma in $\mathrm{M}$ oltre $p .313$ a seguivano compatti e senza stacco da $313 b$ a 317 a altri due Lelii CCLV e CCXLIX, il corrispettivo di questi fra gli Oradini CCLIX ( $i$ sonetti sono, mutato indirizzo, variazione stretta l'uno dell'altro: Mirate, Lelio, ove quei verdi et alti, Mirate, Lucio, ove quell'alta e verde $e$ cosi di seguito), due Oradini della seconda parte, proposta e risposta, CXLVI e CXLVII, e un altro della prima cCLVIII. Unici spersi, il CCLX a p. 327b, e, a p. 329a-b, CCLVI e la proposta della par. $2^{a}$ XVI, scancellata e ricopiata con la risposta a p. 334. Infine a margine di CCLVII ( $p$. 3Iza) è annotato: «questo ha esser l'ultimo di quei di Lelion, accantonando, se non era casuale, l'idea di far convergere le due squadre sul contrappunto di CCXLIX e CCLIX. Certo è che questa volta il deposito, almeno quello di $\mathrm{M}$, è usato come uno spazio per le prove. $\mathrm{M}^{\mathrm{I}}$ inseriva tutti quelli che saranno della prima parte a p. II2b dopo CCXLVII a Giovambattista Tedaldi, che, chiamando in causa il Bonsi ( Deh, come volentier vosco e col mio / BONSI, cui tanto già Minerva deve, / Colà verrei, TEDALDO, ove 'l bel Sieve / Accresce l'Arno con non picciol rio», I-4), offriva l'aggancio: "Qui vanno i nove sonetti, cioè qui sotto Deh come, Non sa, Lelio [CCXLVIII] a 310 a 314 [si dovrà intendere $i$ nove da 310 a a 3I4a, fino 
a CCXLIX] e 319 [aggiunto in interlinea; cioè CCLVI] e poi $i$ due Mirate, Lucio a 314 [CCLIX] e Lucio a 317 [CCLVIII] e poi Lucio a 327 [CCLX]»; seguiva inchiostrato e reso illeggibile: «e poi Lucio a ...» (ripetizione per errore o si riferiva alla risposta di par. $2^{a}$ CXLVI a p. 3Is?).

In $\mathrm{t} 5 \mathrm{~s}$ oltre la zona mediana di tono epistolare s'è visto che riprendevano $i$ segmenti consistenti, ben riconoscibili e nettamente definiti col CCCXIII: CCCXIIICCCXX Farnesi, CCCXXI-CCCXXX Mendozzi, CCCXXXI-CCCLI Ducali. Nemmeno questi avevano ancora preso posizione in N. I Farnesi, tuttavia, erano bell'e pronti nel suo deposito (pp. 296b-300a) quasi nell'ordine definitivo: CCCXIII-CCCXVII, CCCXIX, CСCXX, CCCXVIII, e entravano in quest'ordine in $\mathrm{N}^{\mathrm{I}}$ (230-37) solo anticipato il CCCXVII in penultima posizione; e cosi furono trascritti in $\mathrm{M}$ ( $\not p$. IIsa-II8b). $\mathrm{M}^{\mathrm{I}}$ ha un parziale ripensamento, annotando l'incipit di CCCXIII, che si rivolge a Fiesole, a p. I4, come primo dei monti, $e$ scrivendo a p. IIsa, sul margine «I४», ma senza toccare il sonetto; che, difatti, qui alla fine rimase.

Anche dei Mendozzi alcuni, non tutti e non tutti riuniti, aspettavano nel deposito di N: CCCXXI a p. 355a, CCCXXIX a p. 357b, cCCXXX e CCCXXV alle pp. 358b-59a, СССХХVI, СССХХVIII, СССХХVII alle pp. 363b-64b. E tutti, anzi due e una proposta in più, dovranno aspettare anche in quello di $\mathrm{M}$, senza entrare in ordine, col primo CCCXXI a p. 34Ia, i quattro seguenti CCCXXIICCCXXV riuniti alle pp. 344a-46b, racchiudendo anche la proposta al cardinal Mendoza e la risposta della par. $2^{a}$ LXIV (pp. 344b-45a), a p. 35 Ia il CCCXXIX, ap. $352 a$ il CCCXXX, alle pp. 356a-57b CССXXVI, CССXXVII, la proposta della par $2^{a}$ LXXVI a Domenico Venier, invitato, 9-II, a dedicare tempo e poesia al cardinale, anche avvertendo che «la risposta di questo è a 360», СССXXVIII. Anche il primo dei Mendozzi, a Fiesole era proposto da $\mathrm{M}^{\mathrm{I}}$ a p. I4 di seguito al CCCXIII allo stesso modo e con lo stesso esito.

Dei ducali la gran parte stava insieme già nelle carte finali di $\mathrm{N}$ (pp. 30Ia-309b) nell'ordine CCCXXXII, CCCXLV, CCCXLIII, CCCXXXIII-CCCXXXV, СССХХХVII, СССХХХVIII, CССХLVII, СССХLVIII, СССХХХVI, СССХL, CCCXXXIX, CCCXLVI, CCCXLII, CCCXLIX, CCCL, erano trascritti in ordine in altro contesto il CCCXXXI al cameriere Sforza Almeni a p. Iosb, il CCCXLIV per la morte di Maria Salviati a p. 40a, nei morti. $\mathrm{N}^{\mathrm{I}}$ li inseriva in ordine, numerando 237-43 (237 ripetuto: era già assegnato al CCCXX) i CCCXXXIICCCXXXVIII, 244 il CCCXLII, 245 e 246 il CCCXL e il CCCXLI, 247 il CCCXLIII, 248-53 $i$ CCCXLV-CCCL $e$ in questo numero e successione li registrava $\mathrm{M}$ alle pp. IIga-27a. S'è visto che CCCXXXIX, per una malattia del duca, era stato spostato nel gruppo su questo argomento e che lo recuperò a questo $\mathrm{t} 5 \mathrm{5}$, che aggiunse in fine il CCCLI. Prima della svolta decisa ai sonetti pastorali, che in $\mathrm{t} 5 \mathrm{~s}$ saranno preceduti da questo esplicito titolo, $\mathrm{M}$ inseriva il sonetto al Sonno LXXXVI (7. I27b). La scrittura, più serrata, lo isola in modo netto: potrebbe 
essere inserito da ultimo su spazio bianco. In $\mathrm{N}$, subito prima di questo passaggio ci sono due sonetti di corrispondenza seguiti da risposta del Varchi: par. $2^{a}$ CLXIX e CLXXIX; ma s'è visto che si tratta o di due coppie trascritte provvisoriamente in una carta lasciata bianca o degli ultimi della sezione centrale, dalla quale in esso si passa direttamente ai pastorali.

La cui serie consisteva qui, pp. IIIa-23b, di CCCLII-CCCLXIV, CCCLXXIV, CCCLXVII-CCCLXIX, CCCLXXII, CCCLXXI, CCCLXV, CCCLXVI, CCCCXCVI, CCCLXX, CCCLXXV, CCCLXVI, CCCCXCVI, CCCLXXIII, CCCLXXVI. N ${ }^{\mathrm{I}}, 254-79$ (non si dà conto di certi suoi piccoli incidenti di numerazione) e $\mathrm{M}, p p$. I28a-4I b, l'assumevano, ritoccando oltre CCCLXIV l'ordine e collocandovi $i$ due sonetti epitalami CССLХХVII e CССLХХVIII $\left(\mathrm{N}^{\mathrm{I}} 268,269, \mathrm{M}\right.$ p. 135a-b) da $\mathrm{N}$ (pp. $28 b$ e 29a): CCCLII-CCCLXV, CCCLXXVII, CCCLXXVIII, CCCLXVI-CCCLXIX, CCCLXXI, CCCLXXII, CCCLXX, CCCCXCVI (cassato in M), CCCLXXIIICCCLXXVI. Dei rimanenti e successivi sonetti della prima serie pastorale di $\mathrm{t} 55$, tutti inattestati in qualsiasi parte di $\mathrm{N}$, uno, CCCLXXIX, viene dalle carte finali di M (p. 360b); due, СССLХХХVI e CССLХХХИIII, da c. 95ra-b; un quarto, CCCXC, da c. $98 \mathrm{ra}$ (carte, queste, interne alla parte riservata alla corrispondenza poetica con risposta o proposta, che nella descrizione del codice si sono viste isolarsi e sulle quali si tornerà); due altri ancora, CCCXCII $e$ CCCXCIII, aprivano in $\mathrm{M}(\not p$. 64b-65a) la sezione centrale a carattere e tono epistolare; un buon numero aspettava in quelle carte iniziali, I-VIII, di $\mathrm{M}$, non numerate a pagina, usate come quelle finali per deposito, forse secondo deposito, frammisto a sonetti di corrispondenza con risposta o proposta nell'ordine: ССССХVIII, ССССХVII, par. $2^{a}$ XXIX risp., CССLXXX, CCCLXXXIV, CССXCV, CCCLXXXV, CCCXCIV, par. $2^{a}$ XCIX, CCCLXXXIII, CCCLXXXIX, par. $2^{a} \mathrm{LXV}$, par. $2^{a}$ XIX, par. $2^{a}$ CI, CCCLXXXI, CCCLXXXVII, CCCXCVI, CCCLXXXII, par. $2^{a}$ XXV, Varchi immortal, che quanto sete raro di Giulio della Stufa e risposta Ch'a voi, Stufa gentil, ch'a paro a paro, par. $2^{a}$ XXX, CCCXCI. Di tutti gli altri, CССXCVI-CСССXVI e CCCCXIX-CСССXX, come dell'intera seconda serie intitolata Sonetti pastorali a messer Giovanvettorio Soderini non trovo traccia in ordine né nei depositi cosi di $\mathrm{N}$ come di $\mathrm{M}$. Si tocca qui il punto di maggior distanza fra $\mathrm{M}$ (a maggior ragione $\mathrm{N}$ ) e $\mathrm{t} 5 \mathrm{5}$, per la cui costruzione si deve presumere un consistente apporto da alte carte.

Di seguito ai pastorali in $\mathrm{N}$ ci sono alcune pagine, I24-28, con materiale vario: par. $2^{a}$ CLXXIV proposta e risposta p. I24, CCXCIX p. I25a, CCXCV p. $I 25 b$, cassato e riscritto in altra versione, quella che sarà a stampa, di seguito, p. I26a, CCXCIII p. I26b, CCXX p. I27a, par. $2^{a}$ LXIII proposta p. I27b, CLXXXI p. I28a, CCLXVII p. I28b che, come detto, porta a margine l'annotazione autografa: «ultimo». L'impressione è che siano sonetti appoggiati qui in modo provvisorio alla fine di quella che sarà e che già in $\mathrm{N}$ si configurava come la prima parte. Alcune peculiarità della scrittura e dell'impaginazione avevano 
fatto pensare, descrivendo il codice, che potessero essere carte restate bianche riempite in un secondo tempo. Ora si puì dire una cosa scontata e certa, che quando si decise di cassare la prima versione di CCXCV, $p$. I2sb, e riscriverlo in una seconda, la seguente p. I26 era libera, se poté ospitarla. Solo il CLXXXI sarà considerato dal riordinamento di $\mathrm{N}^{\mathrm{I}}$ col numero 181 , che, però, è assegnato anche al CCXVIII (che è a p. Iobb di N). Tenendo conto del posto che $i$ due sonetti occuperanno in $\mathrm{M}$, ci si sarebbe aspettati per il CLXXXI il numero I85. Tutti gli altri, ignorati da $\mathrm{N}^{\mathrm{I}}$, saranno collocati in ordine direttamente da $\mathrm{M}$, fuor che CCXX, che, deposto a p. 298 a di $\mathrm{M}$, aspetterà $\mathrm{M}^{\mathrm{I}}$ per essere inserito in ordine, come s'è visto, a p. Iopb. In $\mathrm{M}$ dopo $i$ pastorali è lasciata una pagina bianca, che viene a separare con più nettezza di quanto ora (ma non probabilmente in origine) non appaia in $\mathrm{N}$ ciò che in tutt'e due $i$ codici segue, vale a dire $i$ sonetti di corrispondenza accompagnati da risposta $o$ proposta e che costituiranno $\mathrm{t} 57$.

Questi già in $\mathrm{N}$, quindi in $\mathrm{N}^{\mathrm{I}}$ e $\mathrm{M}$, hanno la distinzione principale fra le proposte del Varchi seguite dalla risposta ( $\mathrm{N}$ pp. I29-66; $\mathrm{N}^{\mathrm{I}}$ 280-319; $\mathrm{M}$ pp. 143-87) e le proposte d'altri a lui e sua risposta ( $\mathrm{N}$ pp. 173-272; $\mathrm{N}^{\mathrm{I}} 320-402$; M pp. I89-297a). Si sarà notato che fra l'una e l'altra sequela in tutt'e due $i$ codici c'è un intervallo; che non è fatto, almeno ora, di pagine bianche, ma occupate da sonetti di varia natura, d'alcuni dei quali già è capitato di parlare. In $\mathrm{N}$ da p. I67 a p. I72b si succedono par. $2^{a}$ LXXVIII e risposta, par. $2^{a}$ XVIII e risposta, CLI, CCXCI, la risposta di Dionigi Lippi a par. $2^{a}$ XLI, LXXXVIII, ССХСИI, ССХСIV, ХХ, ССХХVIII. Un altro vario drappello si trova in questo codice fra la serie compatta dei sonetti di corrispondenza col Varchi risponditore e la proposta di Lucantonio Ridolfi, Parmi, Varchi, ogni dì con le due risposte, che (ricordo) hanno il nome del proponente e le due $R$ delle risposte della corsiva più calligrafica e rigida diversa da quella dei testi, come avviene nel primo strato del codice, e Hor che due volte contrassegnato dalla nota autografa «ultimo», alle pp. 280a-8Ib, cioè quelli delle pp. 273-79b: par $2^{a}$ CXXXIX (proposta e risposta), CCXLVI, CCXLVII, LX, XIII, par. $2^{a}$ XXII (proposta e risposta), CLXXVI, XII, CCXXIV, CCXXXI, C, CCXXXII. Non deve ingannare la presenza anche in questi due gruppi di pagine di sonetti con proposta o risposta: s'è visto che esse si isolano o in genere per l'aspetto grafico o perché ci sono (contro al solito) $i$ nomi dei destinatari e dei corrispondenti della stessa mano del testo; s'è potuto, anzi, appurare che il sonetto par. $2^{a}$ LXXVIII fu certamente riscritto in seconda redazione con la risposta a $p .167$, che perciò doveva essere bianca. Si deve allora ribadire il sospetto e quasi trasformarlo in certezza che tutte quelle pagine 167-72 e 273-79 fossero state lasciate bianche, forse in attesa d'altre coppie di corrispondenza, e effettivamente ricevendone alcune, come appunto par. $2^{a}$ LXXVIII, ma anche usate alla stregua di quelle finali che si son dette di deposito. Come almeno per alcuni 
sonetti s'è visto, poco materiale qui depositato fu messo in ordine già da $\mathrm{N}^{\mathrm{I}}$ : par. $2^{a}$ LXXVIII col numero 3I9, par. $2^{a}$ CXXXIX col numero 382; e sono proprio le coppie collocate ciascuna nelle prime pagine, I67 e 273, dei due intervalli che si sono supposti bianchi (anche CCXXIV e CCXXXI, p. 278a-b ebbero $i$ numeri 272, mal leggibile, e 278, poi depennati). Altri furono trascritti in ordine direttamente da $\mathrm{M}$ o riposti nel suo deposito, per esserne quasi tutti tratti da $\mathrm{M}^{\mathrm{I}}$. Allora (ciò che importava il più possibile chiarire) questa sezione, che diventerà la seconda parte dei sonetti, $\mathrm{t} 57$, era già nel disegno iniziale di $\mathrm{N}$ destinata tutta e soltanto alla corrispondenza accompagnata da proposta 0 risposta.

Forse non si sarebbe aspettato che analoghi intervalli adibiti a piccolo deposito di materiale, che andrà in ordine solo in $\mathrm{t5} 50$ in $\mathrm{t} 57$, venissero a riprodursi entro la stessa sezione in M. Eppure anche qui, come segnalato poco sopra e prima descrivendo il codice, dopo $i$ sonetti del Varchi seguiti da risposta e prima di quelli preceduti da proposta ce ne sono sei scompagnati: $p$. I88 (=c. 94v) a-b LXVII e LXVIII; c. 95r-v CССLХХХVI, CССLХХХVIII, proposta di par. $2^{a}$ XXIX, LVI. Un secondo intervallo è poco lontano, dopo p. I92 $(=c .97 v)$, a c. 98r-v occupata da CCCXC, proposta di par. $2^{a}$ LXVII, CCCXI, CLXI. Si son dovute indicare le carte e non le pagine, fuor che la prima, perché dopo I88 era stato segnato I89, I90 I9I ..., ma le cifre furono depennate, I9I sostituita con I89 e cosi via, saltando le due facciate di c. 95. L'altra carta occupata da sonetti scompagnati fu subito saltata dalla numerazione a pagine. Si volle segnalare che $i$ testi di quelle carte erano fuori ordine? Si tratta di sonetti scritti in tempi diversi, come dimostra il carattere della scrittura e la sua disposizione sulla pagina, avvertiti nel descrivere il codice, su carte lasciate bianche per della corrispondenza poetica, che poi non vi fu depositata? Probabilmente l'una e l'altra cosa.

Isolandosi con netterza in tutt'e due i codici ciò che alla sezione non pertiene, di questa si osserverà che a aprirla in $\mathrm{N}$ (par. $2^{a}$ I-III) come in $\mathrm{N}^{\mathrm{I}}$ e $\mathrm{M}$ (par. $\left.2^{a} \mathrm{I}-\mathrm{IV}\right)$ fino, come illustrato all'inizio, in $\mathrm{t} 57$ tocca a sonetti inviati a Annibal Caro, al quale si rivolge in tutt'e quattro le medesime fasi anche il primo dei pastorali. La posizione in vista goduta fin da principio dall'amico vorrà riconoscere anche il legame stretto da lui intrattenuto con Lorenzo LenziLauro come precettore. E, come in $\mathrm{t} 57$, agli scambi di sonetti col Caro, che trattano dell'amore per il Lauro, già in $\mathrm{M} e \mathrm{~N}^{\mathrm{I}}$ ne seguono subito due (par. $2^{a}$ Ve VI) e uno in $\mathrm{N}$ (par. $2^{a} \mathrm{~V}$ ) col Lauro stesso; nel cui nome, dunque, fin dall'origine la sezione di corrispondenza con risposte e proposte, che diventerà 5 5, s'apre. A chiuderla era in $\mathrm{N}$ e in $\mathrm{M}$ la proposta di Lucantonio Ridolfi con due risposte del Varchi. E cosi forse sarà da intendere anche in $\mathrm{N}^{\mathrm{I}}$, seppure, posto il numero 402 alla seconda risposta, né la prima né la proposta ce l'hanno; tuttavia il 40I, che le toccherebbe, non mi resulta assegnato a 
nessuno. Invece né in fine né in altra parte di 557 , tantomeno di $\mathrm{t} 55$, ci sarà traccia di questo particolare e finale scambio di corrispondenza. Eppure anche la prima risposta al Ridolfi cominciava nel nome del Lauro: L'arbor gentil che forte amai molti anni; e la rima A riproponeva due parole in rima, anni $e$ danni, di I, che è tale da $\mathrm{N}^{\mathrm{I}}$. La causa che avrà fatto sparire, non spostare, questa corrispondenza sarà altra e sostanziale; ma nella prospettiva del libro unico ribadire quel richiamo e far risonare la stessa rima e le stesse parole in rima aveva un effetto forse non primario, ma apprezzabile, che nell'autonomia della seconda parte non aveva più ragion d'essere. Anche il Molza, che in $\mathrm{N}$ p. $173, \mathrm{~N}^{\mathrm{I}}$ numero 320, $\mathrm{M}$ p. I89, apriva la sequela dei sonetti d'altri al Varchi, come degno corrispettivo del Caro, cominciava con lo stesso, forse ovvio, richiamo: "Mentre che lieto vi godete all'ombra, / Varchi, del vostro casto amato Lauro ...». In $\mathrm{t} 57$ questa corrispondenza recederà al centocinquantesimo posto a vantaggio d'un amico di giovinezza, Lodovico Martelli, e della sua memoria, che permette lo stesso di cominciare nel nome dell'alloro: "Se quella virtuosa altera fronde ...» (par. $2^{a}$ LXXX I), benché ancora non fosse il Lauro amato e cantato dal Varchi, ma la più generale insegna dei poeti e facesse, nel caso, piuttosto da premonizione. Insomma, una volta che questa sezione del libro unico di $\mathrm{N}$ e $\mathrm{M}$ diventò la seconda parte dei sonetti in libro separato, $\mathrm{t} 57$, la presenza del Lauro, non in generale, ma nei punti strategici, sembra attenuata. Il suo libro in forma di canzoniere era l'altro, t5 5; questo è principalmente l'epistolario poetico.

Entro questi estremi e posta la distinzione di base, $\mathrm{N}$ rivela qui un ordine ancora parecchio provvisorio. Nemmeno vi erano sempre riunite in unica serie le corrispondenze con la stessa persona. Dopo, per esempio, i primi tre sonetti mandati al Caro (sempre s'intenda, anche se d'ora in poi non importerà più dichiararlo, colle risposte) alle pp. I29-3I il quarto, che $\mathrm{N}^{\mathrm{I}}$ metterà in successione, si trovava lontano a $p$. I60, seppure una nota a margine già disponeva il ricongiungimento «cogli altri al medesimo». Similmente il sesto al Lenzi, che seguiva a p. I6I, era indirizzato "cogli altri». E si sarebbe dovuto dire: con l'altro, perché un altro solo, il quinto è, e rimarrà, inviato al Lauro, p. I32: segno, appunto, di quanto vago e aperto fosse lo stato di $\mathrm{N}$ per questa sezione. Simile riunione è comandata per $i$ sonetti di Michelangelo Vivaldi, par. $2^{a}$ XCIV di p. 236 e XCVIII di p. 272, rispettivamente prima e dopo quello di p. 26I, XCVII; cosi per quello del Bronzino, par. $2^{a}$ CXIII di p. 27I, dopo al CXII di p. 244. Né ci sono sempre avvertimenti del genere: non per l'altro del Bronzino, par. $2^{a}$ CXI che si trova a p. 230; eppure alla lettura questi tre e anche il quarto (par. $2^{a}$ CXIV), che pur apparirà solo in $\mathrm{t} 57$, appaiono strettamente collegati e conseguenti; non per $i$ sonetti del Lasca dislocati alle pp. 2IO-II, par. $2^{a}$ XCII-XCIII, a p. 229 LXXXVIII, e alle pp. 240-42 LXXXIX-XCI; non per $i d u e ~ d i$ Trifone Benci, par. $2^{a}$ CXCI e CXCII, uno a p. 233 e l'altro a p. 270. 
In $\mathrm{N}^{\mathrm{I}}$ anche questi si riuniscono e si determina qualche altra superiore aggregazione, come di par. $2^{a}$ LXXVII a Giovanni della Casa e LXXVIII a Francesco Nasi, che al Casa si riferisce, e perciò si succedono coi numeri zI 8 e 319, mentre in $\mathrm{N}$ si trovavano vicini, ma separati, alle pp. I62 e I67. Ben è vero che la trascrizione di par. $2^{a}$ LXXVIII con la risposta in $\mathrm{N}$ è resultata tardiva e perciò sistemata dove era possibile in pagine lasciate bianche. Cosi par. $2^{a}$ XXXVII al Berni, che in N si trovava a $p$. I36 fra il IX a Giovambatista Strozzi e il $x$ a Ugolino Martelli, n'è tolto, lasciandoli strettamente congiungere, e col numero 294 andrà a precedere il XXXVIII a Pietro Aretino; e cosi diversi altri casi; ma non tutti. Difatti, se le risposte al figliolo (Battista Alamanni par $2^{a}$ CV e CVI) erano unite con quelle al padre (Luigi CVIII-CX) fin da $\mathrm{N}(\not p .174-78)$ e rimarranno in $\mathrm{N}^{\mathrm{I}}$ (numeri 32I-25) e in $\mathrm{M}$ ( $p$. 190-94) $e$ in $\mathrm{t} 57$, aggiungendosi CVII (Battista), non altrettanto semplice cura ebbe per le proposte a questo (par. $2^{a}$ LIX, LX) e l'unica, per allora, a quello ( $L X I I) \mathrm{N}$, che le scrisse alle pp. I40-4I e I64, ma nemmeno $\mathrm{N}^{\mathrm{I}}$, che le segnò dei numeri 300-30I e 314, né M, che le lasciò separate alle pp. 163-64 e 179; ci pensò solo $\mathrm{t} 57$ che anche trasse l'altra proposta e relativa risposta di Battista, par. $2^{a}$ LXI, dai depositi di N (pp. $348 b$ e $\left.357 a\right)$ e di M (pp. 33Ib e 350b). Poco o nulla per questa sezione $\mathrm{N}^{\mathrm{I}}$ andrà a pescare dal deposito di $\mathrm{N}$ : par. $2^{a}$ XVII a Lelio Bonsi, da p. 3II, riunendolo col numero 305 agli altri due per lui, che soli $\mathrm{N}$ schierava in ordine alle pp. Is I e Is2. Qualcosa di più M, ma non tanto. Senza entrare in particolari, basterà tener conto che dai centoquarantuno scambi di sonetti di $\mathrm{N}$, calcolando anche par $2^{a}$ LXXVIII e CXXXIX inseriti in pagine bianche (I67 e 273) in tempo perché $\mathrm{N}^{\mathrm{I}}$ potesse considerarli, e scesi subito a centotrentanove, scancellati par. $2^{a}$ CLXVI a $p .188$ (recuperato da $\mathrm{M}$ a p. 295) e CII a p. 212 (sarà recuperato da $\mathrm{M}^{\mathrm{I}}$ a p. 26I), $\mathrm{M}$, che ne lascerà cadere un altro, quello col Guglia, di $\mathrm{N}$, p. $228\left(\mathrm{~N}^{\mathrm{I}}\right.$ 352), Varchi, l'invitto vostro alto valore $e$ Guglia, c'habbia di me nel vostro core (non più recuperato), arriverà a ordinarne centocinquantatré, attingendo sia al deposito sia richiamandone qualcuno disseminato in $\mathrm{N}$ nelle precedenti sezioni. Ben più lungo sarà il passo di t5 7 sia nell'organizzazione, su cui s'è fatto qualche cenno in principio, sia nell'estensione, raggiungendo dugentodiciannove scambi, in piccola parte anticipato da $\mathrm{M}^{\mathrm{I}}$ che ne inserisce nove: par. $2^{a}$ LXIII $p$. I60, XVI p. I68, LV p. I75, CLXXXIX p. 229, CII p. 26I, CXLVI e CXLVII p. 280, CСXV e CСXVI pp. 294 e 295. Solo due saranno abbandonati da $\mathrm{t} 57$, il sonetto di Benedetto Cipello, Quell'aura onde Helicona il vostro altero e le due risposte Ben m'è caro e giocondo, ma nel vero $(\mathrm{M} p$. 2Iб) e Caro Cipello mio, se così vero $(\mathrm{M} p .327) . \mathrm{M}^{\mathrm{I}}$ aveva prima ricongiunto la seconda risposta alla prima a p. 216, quindi spostato tutto a p. $295,{ }^{18}$ prima dell'altra proposta, di Lucantonio Ridolfi, con due risposte; anche queste abbandonate da $\mathrm{t} 57$. 
La lezione, invece, tende a restare stabile da $\mathrm{M}$ a $\mathrm{5} 57$, mentre era in tanti sonetti mutata fra $\mathrm{N}$ e M, o, meglio, in $\mathrm{N}$, su cui sono fatte la gran parte delle correzioni. Ma su queste, come promesso sopra, non ci s'intrattiene, se non per rilevare che la penna del Varchi s'esercita non solo sui propri ma anche sui sonetti altrui. Si ritoccano in modo anche esteso risposte o proposte del Lenzi, del Bonsi, dell'Oradini e di tanti altri. Qualche esempio: nella risposta del Lenzi, par. $2^{a}$ V 8 (N p. I32) "Volga le spalle per contrario vento» è corretto dalla corsiva del Varchi in «s'arreste, o tema invidioso vento»; in una di Lelio Bonsi, par. $2^{a}$ XV F (N p. I52) «Per questo, ch'io ho preso alpestro, e fero / Cammin» è mutato in «Per questo, che preso ho spinoso, e fero / Cammin»; nella proposta di Lucio Oradini, par. $2^{a}$ CXLV 3-4 (N p. 254) «Tale ha beltate in sé, tanta honestate, / Ch'a tutte l'altre, ogni altra lode involas, dove beltate faceva rima interna con $B$ e altra bisticcio con altre, è corretto in "Tale ha bellezza in sé, tanta honestate, / Ch'a tutte l'altre, ogni alta lode invola». Con questi più giovani amici si può capire che per il Varchi fosse difficile in qualsiasi momento lasciare $i$ panni del maestro. Lo stesso può valere anche verso il coetaneo Bronzino, che a lui sembra guardare con altrettanta reverenza di discepolo, sicché all'incipit di par. $2^{a}$ CXI «Varchi, ch'a par de' più rari e migliori», accettò di buon grado la variante «saggi»» (prima s'era proposto «dotti» N p. 230) e cosi il sonetto comincia nel codice delle sue rime, II IX Io della Nazionale di Firenze, c. 33v. Appare meno facile da comprendere che non si tenesse a freno la penna davanti alla risposta, par. $2^{a} \times(\mathrm{N}$ p. 137$)$ d'un personaggio più giovane ma poeta rispettato come Ugolino Martelli, cui, invece, è rifatto un verso (8, ma pare coinvolta tutta la giuntura di 7-8): "Voi'l devete acquetar col vostro CARO / Cui meco tanto a tal ragion amate», che diventa: "Voi'l devete acquetar col vostro CARO / Annibal, ch'a ragion si forte amate». Ma anche alla risposta dello stesso Caro, par. $2^{a}$ I II (N p. I29), la «Fortuna ingiuriosa» diventa a margine "Destino invidioso». Qui la mano che corregge invece che l'inconfondibile corsiva del Varchi è quella più rigida e calligrafica, ma che (sia sua o d'altri) scrive di certo sotto il suo controllo. Se in teoria il Caro avrebbe potuto approvare o anche proporre la variante, il Molza non più. La sua proposta, par. $2^{a}$ CL (N p. I73) leggeva ai vv. I-2: "Mentre che lieto vi godete all'ombra, / Varchi, del vostro caro (?) amato (?) Lauro», e la corsiva del Varchi mutò l'aggettivazione in «casto amato». Si sa quanto la castità dell'amore per il Lauro fosse essenziale e irrinunciabile alla raccolta dei sonetti. La correzione dice che a far aggio su tutto è l'unità e coerenza del libro, che significa una chiara e unitaria impostazione e responsabilità, anche quando vi s'inglobano cose d'altri.

Ritornando al libro, dei due codici preparatori, o ai libri, delle stampe del I5ss e I557, e provando a tirare un po' le fila, la gestazione, come s'è visto, fu una 
sola e il parto, per continuare la metafora, gemellare, anche se i gemelli videro la luce a distanza di due anni, per nulla eccessiva (fuori metafora). I due manoscritti considerati hanno permesso di seguirla da una fase già abbastanza avanzata, quella della trascrizione in ordine di $\mathrm{N}$, che sempre più è venuta a identificarsi, determinandolo a sua volta, con quello che da indizi esterni, grafici e d'impaginazione, appare il primo strato della sua scrittura, fino al I553-54. Questa data si cava dal sonetto Hor che due volte, che nell'uno e nell'altro suggella la sezione di quelli scritti in ordine ( $\mathrm{N} p .28 \mathrm{I} b, \mathrm{~N}^{\mathrm{I}}$ numero 403 e M p. 297b) e che corrisponde nella sostanza e ufficio, benché nella formulazione sia tutto diverso, al DXXXIV, riportato sopra, conclusivo della prima parte o t5 5. Ecco il sonetto nei due codici, un sonetto che per il posto e la funzione che ha merita d'esser considerato un po' di per sé, senza precipitarsi e tuttavia arrivando ben presto alle deduzioni cronologiche che offre:

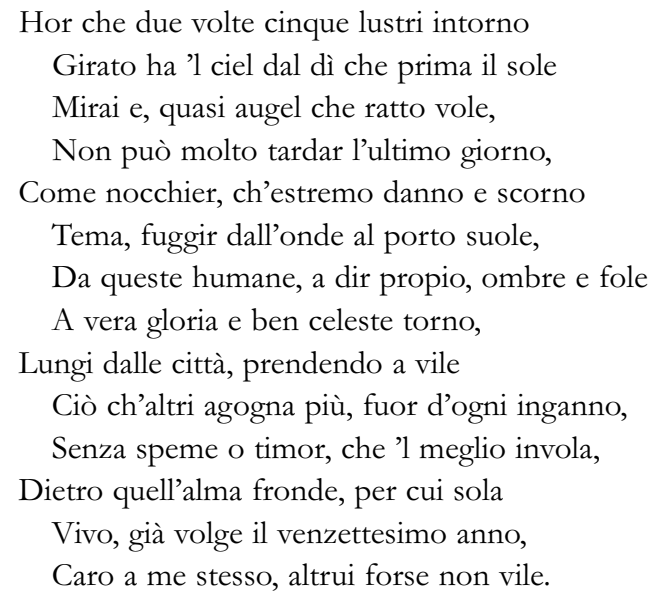

Questo il testo base, identico nei due manoscritti, non toccato in $\mathrm{N}$, mentre in $\mathrm{M}$ tutto il $v$. 8 è sostituito a margine con «A te, vero del ciel Signor, ritorno», il secondo emistichio di Io con «fuor d'ira e d'inganno», a I3 «volge» con «cade». Le prime due correzioni sono della scrittura del testo, la terza della corsiva tipica del Varchi. Nessuna incide sul tono generale, che è sempre quello del congedo e consuntivo, che sarà del DXXXIV in $\mathrm{t5} 5$. Come quello chiuderà la prima parte dei sonetti, quella che sola nelle stampe assume $i$ tratti del canzoniere, cosi questo chiude in $\mathrm{N}^{\mathrm{I}}$ e in $\mathrm{M}$ il libro unico dei sonetti che anche s'apriva con I, tipico testo iniziale di canzoniere. In $\mathrm{N}$ l'uno e l'altro appaiono aggiunti, in tempo, tuttavia, per il riordinamento di $\mathrm{N}^{\mathrm{I}}$. A quell'altezza, dunque, il canzoniere era quell'unica lunghissima raccolta di sonetti trascritti in ordine nei due codici, comprensiva di un'estesa sezione di corrispondenze accompagnate da risposta o proposta, che andrà a costituire 5 5. In Hor che 
due volte c'è di più e di diverso rispetto a DXXXIV un accenno di contrizione e proposito di mutar vita (5-II), che non coinvolge, e non poteva coinvolgere per le ragioni dette all'inizio, l'amore per il Lauro; anzi, la conversione è «Dietro quell'alma fronde» (I2). Nelle correzioni di $\mathrm{M}(8)$ il sonetto di tono spirituale, venendo a rivolgersi a Dio, diventerà esplicita preghiera e mostrerà $i$ segni ancora più palesi, forse scontati, della conclusione d'un percorso lirico che voglia essere unitario, per intendersi convenzionalmente, d'un canzoniere. In ts 5 la preghiera sarà, è parso per coerenza, solo di ringraziamento e messa in principio, a II, un sonetto che entrerà in $\mathrm{N}^{\mathrm{I}}$, ma che la trascrizione in ordine di $\mathrm{N}$ ignorava.

Dunque la chiusura in $\mathrm{N} e \mathrm{M}$ del libro onnicomprensivo, Hor che due volte, porta due riferimenti cronologici. L'autore ba compinto $i$ cinquant'anni: «due volte cinque lustri intorno / Girato ba 'l ciel dal dì che prima il sole / Miraì (I-3); essendo nato nel marzo I503, doveva aver passato il marzo I553; e questo è univoco. Rispetto all'incontro col Lauro si parla di ventisette anni: "rià volge [poi cade] il venzettesimo anno». L'evento è fissato dal sonetto XXXVI (9-II) alla fine d'agosto del I527: "Ventisette anni e cinquecento havea / Dopo il mille girato il sole ed era / Nel quinto grado della bella Astrea». Per dire che volgeva o cadeva il ventisettesimo anno dall'agosto del I527 probabilmente bastava che quell' anno fosse in corso, cioè si fosse passato l'agosto I553. Se si volesse intendere che ventisette anni erano compiuti, bisognerebbe aspettare la fine d'agosto del I554, quando il Varchi ne contava ormai piu di cinquantuno, anche se nulla l'obbligava a precisarlo e che n'avesse passati cinquanta rimaneva comunque vero. Sembrerebbe, tuttavia, un po' da preferire la prima ipotesi, anche perché in $\mathrm{N} e$ in $\mathrm{M}$ è presente, ultimo effettivo della serie dei morti, il sonetto CLV per la perdita di Pierino da Vinci, avvenuta, pare, nel I553, ma in nessuna parte di nessuno dei due compaiono CLXIV e CLXV, che piangono quella di Gaspara Stampa, 23 aprile I554, e entrano solo, a chiudere la serie, in $\mathrm{5} 5$. Anche questa data, certo, è un terminus post quem $e i$ due sonetti di per sé potrebbero essere stati scritti dopo diversi mesi; sicché la loro assenza non può collocare $\mathrm{M}(e \mathrm{~N})$ prima di quel giorno preciso, piuttosto e verosimilmente o prima o non tanto dopo. In ogni modo, ricordato ancora che I e Hor che due volte sembrano proprio aggiunti in $\mathrm{N}$ e precisato, quindi, che il corpo del libro vi fu sbozzato prima della data ricavabile da questo sonetto (ma s'è visto ora che CLV, trascrittovi in ordine a p. 44b, dovrebbe portare già al '53), la definizione del tutto in $\mathrm{N}$, in $\mathrm{N}^{\mathrm{I}}$ e in $\mathrm{M}$ si collocherà dopo il marzo I553, nel corso di quell' anno e del successivo. Il I2 gingno del I5I5, s'è detto in principio, è datata la dedica a Francesco de' Medici di t5 5. E difatti il sonetto finale, DXXXIV, corrispettivo di Hor che due volte, si data e aggiorna la prima parte dei sonetti «al ventottesimo anno» (4) dallincontro col Lauro, alla lettera dall'inizio della 
poesia che n'è scaturita, cioè nel corso di quell'anno, che si sarebbe compiuto solo con gli ultimi d'agosto. Ma anche si sa per certo da una lettera del Varchi che nel febbraio 'ss il libro era in tipografia. ${ }^{19}$ In quei mesi, poco più o poco meno di dodici, maturò la decisione, che sarà stata persuasa anche dalla misura, già grande e che sempre più s'ingrandiva, di separare l'unico libro canzoniere dall'epistolario poetico, che, visibile e definito, v'era stato racchiuso, e di dare a ognuno piena autonomia: l'uno pubblicato a stampa subito, l'altro due anni dopo. Ci si rese conto infine, in quei mesi, che se non s'interveniva sul feto, si sarebbe partorito una cosa, certo, mai vista, ma forse mostruosa, in tutto analoga a quelli che oggi si chiamano gemelli siamesi. I gemelli, invece, poterono cosi venire alla luce debitamente separati, ma gemelli nell'autonomia raggiunta rimasero. Furono chiamati De' sonetti di m. Benedetto Varchi parte prima $e$ De' sonetti di m. Benedetto Varchi colle risposte e proposte di diversi parte seconda, per avvertire di quellintimo e ben visibile legame, che la separazione infine sopravvenuta non aveva cancellato, solo impedito che diventasse reciproco e insopportabile impaccio.

Giuliano Tanturli 


\section{Benedetto Varchi}

I. Al soggiorno accenna anche Umberto Pirotti, Benedetto Varchi e la cultura del suo tempo, Firenze, Leo S. Olschki editore, I 97 I, p. 50, nota 5.

2. I numeri romani coi quali saranno indicati i sonetti, quando necessario preceduti dall'indicazione parte $2^{\text {a }}$ (altrimenti, se non è esplicito dal contesto, s'intende la prima), sono apposti, rispettando il contenuto e l'ordine delle prime edizioni cinquecentine citate, nelle Opere di Benedetto Varchi, ora per la prima volta raccolte con un discorso di $A$. Racheli intorno alla filologia del secolo XVI e alla vita e agli scritti dell'autore, aggiuntevi le lettere di Gio. Battista Busini sopra l'assedio di Firenze, Trieste, Lloyd austriaco, i 85 8-i 859 . Nella seconda parte il numero è comprensivo di proposta e risposta.

3. Cfr. Pirotti, Benedetto Varchi cit., p. 35.

4. Vanni Bramanti, Ritratto di Ugolino Martelli, in «Schede umanistiche», I999, pp. I7 e 29.

5. Sul senso di questa corrispondenza e la circostanza cfr. Silvia Longhi, Della Casa, Varchi, Bembo e la vera gloria (Scheda per il sonetto Feroce spirto), in «Studi e problemi di critica testuale», XIX (I979), pp. I 27-34.

6. La prima parte dei sonetti è studiata da Bernhard Huss, "Cantai colmo di gioia, e senza inganni". Benedetto Varchis Sonetti (parte prima) im Kontext des italianischen CinquecentoPetrarkismus, in «Romanistisches Jahrbuch», LII (200I), pp. I 33-57.

7. Cfr. Pirotti, Benedetto Varchi cit., p. 7.

8. Se è giusta la proposta di Silvano Ferrone, esperto di cose varchiane e fiesolane, in BenedetTo Varchi, Epigrammi a Silvano Razzi, Introduzione, edizione critica con commento e traduzione a cura di S. Ferrone, Fiesole 2003 , p. Io, uno di questi sonetti di ritorno a Fiesole, il XLVII, sarebbe da collocare verso l'estate del i 525.

9. Il cclXi è detto per Lucio Oradini nella tavola dei destinatari, per Lionardo Marinozzi d'Ancona in quella degli incipit.

I . Su questi sonetti del Varchi, sull'opera e altre celebrazioni letterarie cfr. Simone Albonico, Il Ruginoso Stile. Poeti e poesia in volgare a Milano nella prima metà del Cinquecento, Milano, Franco Angeli, I990, pp. 258-59.

I I. Il codice è censito e brevemente descritto, con indicazione sommaria del contenuto e rilevandone le «correzioni autografe», nel volume XI di Giuseppe Mazzatinti e Fortunato Pintor, Inventari dei manoscritti delle Biblioteche d'Italia, Forlì, Casa editrice Luigi Borandini, I 90 I, p. 250.

I 2. In questa peregrinazione, in coincidenza della quale anche fu rielaborata la seconda terzina, ai vv. 9-I 4 prima si oscurò la sintassi e il senso, guastandosi la rima dell'ultimo; infine sintassi e senso furono recuperati, anche se ardui, ma rimase sacrificata la rima. Così le terzine del sonetto in t5s: «Gl'huomini, gl'animai, gl'arbori e l'herbe, / E quanto scalda il sol, d'amare invoglia / Virtù, che 'l terzo ciel benigno piove. / Sol quelle sempre dolci e sempre acerbe, / Cui folgore non tocca, o vento muove, / Non cangian mai color, se mutan, foglie»; si potrebbe sospettare almeno per la rima imperfetta fra Io, invoglia, e I4, foglie, della stampa, che, invece, è incolpevole. In $N$ p. I Ib e in $M$ p. 2 I b, i vv. I 2-I 4 erano: «Sol quelle sempre verdi, e sempre acerbe / Frondi, cui vento mai né folgor muove, / Non cangiano color, né mutan foglia», con sintassi e senso limpidi e rime tutte perfette; ma sul margine e nell'interlinea di $M$ nell'inconfondibile corsiva del Varchi diventarono: «Sol quelle sempre dolci, e sempre acerbe, / Cui folgore non tocca o [corr. su né fulgore mai né] vento muove / Non cangian mai pensier né mutan voglie», e così furono 
trascritti nel deposito di p. 354a. L'autore si sarà reso conto che non c'era un sostantivo cui riferire il pronome femminile quelle; intervenne di suo pugno restaurando sia color al posto del pensier sia foglie al posto di voglie, ma al plurale. La sintassi tornò in sesto con un forte, ma anche mirabile iperbato, se non fosse stato per quel mutan, da prendere come tautologico di cangian, ma che denunciava il rappezzo. Con la stampa si rimediò anche a questo, sostituendo né con se e la coordinata con un'ipotetica. Ma alla rima o non si fece caso o si rinunciò.

I 3. In verità per il v. 9 a p. 356b il testo legge: «Che rimirargli sempre e di lor sempre» (con rimirargli in interlineo), e il margine corregge: «Se non sempre mirargli e di lor sempre», che è la lezione a testo di p. 46b; ciò che indurrebbe a ritenere questa trascrizione posteriore a quella. Ma può benissimo trattarsi di una variante del testo di p. 356 rientrata. D’altra parte è costante l'allineamento della p. $356 \mathrm{~b}$ di $N$, di $M$ e di t5s contro la p. $46 \mathrm{~b}$ di $N$ : vv. 6-8, «così dolce i duoi / Lumi volgeansi, onde a me nulla poi / Non piacque o piacerà poco né molto» $N$ p. 46b, corretto a margine in "piacque, né p. ", "così dolce i duoi / Occhi volgeansi e tal che nulla poi / Mi piacque o piacerà poco né molto» $N$ p. $356 \mathrm{~b}, M$, t55; v. I 2, «E s'a voi il languir mio» $N$ p. 46b, corretto a margine in "penar», «E s'a voi l'arder mio» $N$ p. $356 \mathrm{~b}, M$, t5s.

I4. Il LXXVII 5-I I è così a p. 49a: «Bench'io, poi ch'ogn'hor più m'inaspro e 'nduro / Del duol, cui lungi a voi fo larga strada, / Della mia pena sola, non pur rada / Fra quante hor sono al mondo, e quante furo; / Devrei trovar pietà, ch'asprezza uguale, / $\mathrm{O}$ più selvaggia, e soletaria vita / Non sentì mai né visse alcun mortale», e così a p. 356a: «Ben ch'io poi ch'ogn'hor più m'innaspro, e 'nduro / In questa horrida alpestra, herma contrada / Del duol cui [corr. in interl. su che] lunge a voi fo larga strada / E dall'Arbor che sol nel mondo curo / Devrei trovar pietà, ch'asprezza uguale / Né più selvaggia, o soletaria vita / Non sentì mai, né visse alcun mortale» (che sarà la lezione di 455 ). A p. 52 a il CIII 5 a testo legge: «Se del [corr. a marg. $d$. bel] casto, cortese, alto disio», corretto sul margine superiore: «Se di sincero e ... [parola illeggibile sotto la cassatura] casto disio», quindi: «Se di cortese affetto alto disio», a p. 36ob: «Se d'honesta beltà casto disio»; la sirima è tutta diversa, così a p. 52a: «Ma voi, che sì lontan da terra sete, / E sì vicino al ciel, Voi, che monarca / Nel mezzo del mio cor sempre sedete, / Perché quel che con gl'occhi ogn'hor vedete, / Speme [corr a marg. s. in Alma] d'honesto Amore [corr. a marg. d. in foco], e fede carca, / Per mia morte a voi stesso non credete?», e così a p. $360 \mathrm{~b}$ : «Dunque sarà, che la rea turba, e 'l vile / Stuolo, che contra i Buon sempre bisbiglia / Al maggior ben, che sia quaggiù mi scioglia? / Certo non fia, che non ben si consiglia / Quando per falsa tema un cor gentile / Del suo proprio tesor se stesso spoglia». t5s starà con la versione di p. $360 \mathrm{~b}$.

I 5 . Il XCII 5 a p. 29 b ha subito la lezione definitiva di $N$ a p. 52 a; al v. I 3 «Alma» è ricorretto a margine in «Speme»; sul margine di p. $348 \mathrm{~b}$ è introdotta la correzione «Dal maggior» al posto di «Al maggior».

I6. La lezione del secondo emistichio di ro e di i I, che a testo alle pp. 347 e 353 di $N$, come 333 di $M$ era: «così virtù vera / Vincer le voci del rio volgo suole», alla p. 353 di $N$, soprascrivendo, e in margine di p. 333 di $M$ porta la correzione al v. Io: «sì virtute intera»; la frase riportata così a p. 338 di $M$ fu corretta nel margine nel modo in cui compare anche in t57, cioè: «così salda, e 'ntera / Virtù vincer le voci e 'l volgo suole».

I7. Nelle Due lezioni di m. Benedetto Varchi .... In Fiorenza appresso Lorenzo Torrentino Impressor ducale MDXLIX, alle pp. I 8 -ig i due sonetti erano stati stampati sotto la rubrica: «Due sonetti fatti già quando si scoperse la cappella di San Lorenzo a m. Lorenzo Lenzi e a m. Bartolomeo Bettini»; erano stati stampati, si suppone, perché presentando in $N$ Più non mi par Bettin una correzione all'ultimo verso, da «Perché l'arte non ceda alla Natura» a «per ch'arte vaglia quanto può natura», la stampa ha ancora la versione prima. 


\section{Benedetto Varchi}

I 8. Per la precisione a p. 2 I 6 è annotato: «questo colle 2 risposte si scriva dopo il son. Quanto il contento a 295", a p. 295 dopo il sonetto di risposta a Filippo Valentino, che comincia Quanto il contento, par. ${ }^{a}$ CLXVI è scritto: «Dopo questo va il son. Quell'aura a 2 I6 colla risposta». ¿̀ facile che il singolare risposta sia o una svista o un'indicazione approssimativa, anche se non si può escludere l'intento d'inserirne una sola.

I 9. La lettera, che può leggersi in Benedetto VARChi, Opere, II, p. 828, è datata, secondo lo stile fiorentino, 6 febbraio i 554 (cioè '5 5 ) e si rivolge al Torrentino, dicendo che «In quelle tavole e sonetti che voi mi mandaste a casa, non sono alcuni sonetti che io dei a $\mathrm{m}$. Giovannantonio [...] sì che vi piacerà fare che io abbia il mio libro così sdrucito, che andrò rinvergando il tutto», precisando, tra l'altro, il titolo e il frontespizio, così come di fatto si legge, che intanto l'editore potrà «far ristampare» (dunque in una prima bozza era diverso), così come il sonetto CCXLVIII, indicato col numero della carta e con l'incipit. Di questo furono corretti solo nell'errata corrige, ossia nella lista degli errori più notabili, posta nell'ultima carta del volume, i vv. 7-8 (l'errata corrige dice, per errore, ottavo e nono) e I 2. Ma si deve avvertire che a 7-8 la correzione da «Non s'accorge la stolta, che sue brami / Non son cosa mortal che tosto passa» a «Ahi stolta, e non t'accorgi quel che brami / Esser cosa mortal che tosto passa», che sembra opportuna quant'altra mai, si applica non solo alla p. I 26 di t55, ma anche a $M$ e a $N$ e quindi, andrà supposto, al testo mandato in tipografia. E questo a discarico d'almeno una delle tante colpe riversate sui tipografi. L'attenzione sull'importante documento mi è stata richiamata da Vanni Bramanti, che ringrazio. 


\section{APPENDICE}

La tavola serve a rintracciare in $N$, in $N^{t}$, in $M$ e $M^{t}$ i sonetti di $t_{55}$ e di $t_{57}$, incolonnati a sinistra, cui seguono in orizzontale le pagine dei codici in cui ciascuno compare e il numero d'ordine assegnatogli in $N^{t}$. La freccia, $\rightarrow$, indica correzioni o spostamenti interni, la sbarra, /, un'alternativa, la lineetta, -, significa che il sonetto non c'è, il numero a esponente l'ordine nel quale un incipit è inserito nella pagina. Le parole in tondo di seguito ai numeri sono annotazioni dei codici. Si pongono in fine gli incipit in ordine alfabetico dei sonetti presenti nei codici e non stampati in 555 o 57 .

\begin{tabular}{|c|c|c|c|c|}
\hline t5s & $N$ & $N^{T}$ & $M$ & $M^{T}$ \\
\hline I & c. IIV & n. I & р. га & \\
\hline II & p. 3I2a & 2 & $\mathrm{Ib}$ & \\
\hline III-IV & $\mathrm{Ia}-\mathrm{Ib}$ & $3-4$ & $2 a-2 b$ & \\
\hline V & $314 \mathrm{~b}$ & 5 & $3 a$ & \\
\hline VI-VIII & $2 a-3 a$ & 6-8 & $3 b-4 b$ & \\
\hline IX & $347 \mathrm{a}$ & - & $330 \mathrm{a}$ & p. $2 \mathrm{Ia}^{\mathrm{I}} \rightarrow 4 \mathrm{~b}^{\mathrm{I}}$ \\
\hline $\mathrm{X}-\mathrm{XI}$ & $3 b-4 a$ & 9-1о & $5 a-5 b$ & \\
\hline XII & $277 \mathrm{~b}$ & - & $304 \mathrm{~b}$ & $5 \mathrm{~b}^{2}$ \\
\hline XIII & $275 \mathrm{~b}$ & - & $303 \mathrm{~b}$ & $5 \mathrm{~b}^{\mathrm{I}}$ \\
\hline XIV & - & - & - & \\
\hline $\mathrm{xV}$ & $334 a$ & - & $324 \mathrm{~b}$ & $102 b^{1} \rightarrow 22 a^{7} \rightarrow 6^{1} a$ \\
\hline XVI & Isa & I 2 & $6 \mathrm{~b}$ & \\
\hline XVII & $34 \mathrm{Ib}$ & - & $333 a$ & $2 \mathrm{ob}^{2}$ \\
\hline XVIII & $32 \mathrm{~b}$ & - & $332 \mathrm{a}$ & $20 \mathrm{~b}^{3}$ \\
\hline XIX & $9 \mathrm{a}$ & 4I & $2 \mathrm{ra}$ & \\
\hline $\mathrm{xx}$ & $172 a$ & - & $305 a$ & $22 \mathrm{~b}^{3} \rightarrow 2 \mathrm{Ia}^{3}$ \\
\hline XXI-XXIV & $5 a-6 b$ & $13-16$ & $7 a-8 b$ & \\
\hline XXV-XVII & $7 b-8 b$ & $18-20$ & $9 \mathrm{~b}-\mathrm{rob}$ & \\
\hline XxVIII & $7 \mathrm{a}$ & I7 & $9 \mathrm{a}$ & \\
\hline XXIX & - & - & - & \\
\hline $\mathrm{xxx}$ & $337 a$ & - & $323 a$ & $\mathrm{I} 3 \mathrm{a}^{\mathrm{I}}$ \\
\hline XXXI & 3 roa & $2 \mathrm{I}$ & I Ia & \\
\hline XXXII & ioa & 28 & I $4 \mathrm{~b}$ & \\
\hline XXXIII & $335 \mathrm{~b}$ & - & $324 a$ & $\mathrm{I} 4 \mathrm{~b}^{\mathrm{I}}$ \\
\hline XxxIV-Xxxy & Iob-ira & $29-30$ & I $5 a-I 5 b$ & \\
\hline xxxvI & $334 \mathrm{~b}$ & - & $325 a$ & $16 \mathrm{a}^{\mathrm{I}}$ \\
\hline XXXVII & $20 \mathrm{a}$ & 4 & $17 \mathrm{~b}$ & \\
\hline XXXVIII & $314 a$ & 37 & iga & \\
\hline XXXIx & $293 \mathrm{~b}$ & 38 & $19 \mathrm{~b}$ & \\
\hline $\mathrm{XL}$ & $359 \mathrm{~b}$ & - & $347 a$ & \\
\hline
\end{tabular}




\section{Benedetto Varchi}

\begin{tabular}{|c|c|c|c|c|}
\hline XLI & $2 \mathrm{ob}$ & 35 & I $8 \mathrm{a}$ & \\
\hline XLII & $3 \mathrm{I} 6 \mathrm{~b} \rightarrow 2 \mathrm{Ob}^{\mathrm{I}}$ & 36 & $\mathrm{I} 8 \mathrm{~b}$ & \\
\hline XLIII-XLIV & I $2 a-I 2 b$ & $32-33$ & I $6 \mathrm{~b}-\mathrm{I} 7 \mathrm{a}$ & \\
\hline XLV & $35 \mathrm{Ia}$ & - & $337 a$ & $\mathrm{Iob}^{\mathrm{I}}$ \\
\hline XLVI & $\mathrm{I} 7 \mathrm{~b}$ & 23 & I $2 \mathrm{a}$ & \\
\hline XLVII & $\mathrm{I} 7 \mathrm{a}$ & 26 & I $3 b$ & \\
\hline XLVIII & $\mathrm{I} 8 \mathrm{~b}$ & 25 & I3a & \\
\hline XLIX & I $8 \mathrm{a}$ & 24 & $\mathrm{I} 2 \mathrm{~b}$ & \\
\hline $\mathrm{L}$ & $3 \mathrm{Iob}$ & 27 & I $4 \mathrm{a}$ & \\
\hline LI & $293 a$ & 40 & $20 \mathrm{~b}$ & \\
\hline LII & $337 \mathrm{~b}$ & - & $332 \mathrm{~b}$ & $20 b^{\mathrm{I}}$ \\
\hline LIII & $\mathrm{I}_{3} \mathrm{~b}$ & 43 & $22 \mathrm{a}$ & \\
\hline LIV & $339 \mathrm{~b}$ & - & $320 \mathrm{a}$ & $22 \mathrm{a}^{\mathrm{I}}$ \\
\hline LV & $339^{a}$ & - & $320 \mathrm{~b}$ & $22 a^{2}$ \\
\hline LVI & - & - & c. $95 \mathrm{vb}$ & \\
\hline LVII & $340 \mathrm{a}$ & - & p. $32 \mathrm{Ia}$ & $22 a^{3}$ \\
\hline LVIII & I $4 \mathrm{a}$ & 44 & $22 \mathrm{~b}$ & \\
\hline LIX & $335 a$ & - & $323 \mathrm{~b}$ & $22 \mathrm{a}^{8} \rightarrow 22 \mathrm{~b}^{\mathrm{I}}$ \\
\hline $\mathrm{LX}$ & $275 a$ & - & $303 a$ & $22 \mathrm{~b}^{2}$ cass. \\
\hline LXI & I $4 \mathrm{~b}$ & 45 & $23 a$ & \\
\hline LXII & $285 \mathrm{~b}$ & 46 & $23 \mathrm{~b}$ & \\
\hline LXIII & $4 b$ & I I & $6 a$ & \\
\hline LXIV & $336 \mathrm{~b}$ & - & $322 \mathrm{~b}$ & $22 \mathrm{a}^{6} \rightarrow 2 \mathrm{Ia}^{2}$ \\
\hline LXV & $34 \mathrm{ob}$ & - & $32 \mathrm{Ib}$ & $22 a^{4}$ \\
\hline LXVI & $336 a$ & - & $322 a$ & $22 a^{5}$ \\
\hline LXVII-LXVIII & - & - & I $88 \mathrm{a}-\mathrm{I} 88 \mathrm{~b}$ & \\
\hline LXIX & $29 \mathrm{Ib}$ & 72 & $6 \mathrm{~b}$ & \\
\hline LXX-LXXI & $2 \mathrm{Ib}-22 \mathrm{a}$ & $5 \mathrm{I}-52$ & $26 a-26 b$ & \\
\hline LXXII & $2 \mathrm{Ia}$ & 50 & $25 \mathrm{~b}$ cass., & \\
\hline LXXIII-LXIV & $45 \mathrm{a}-45 \mathrm{~b}$ reint. & $48-49$ & $24 \mathrm{~b}-25 \mathrm{a} c$ & int. \\
\hline LXXV & 46a cass. e reint. & 47 & 24 a cass. $e$ & \\
\hline LXXVI & $46 \mathrm{~b}$ cass., $356 \mathrm{~b}$ & - & $348 b$ & \\
\hline LXXVII & 49a cass., $356 \mathrm{a}$ & 60 & $3 \circ \mathrm{ob}$ cass. & \\
\hline LXXVIII & $22 \mathrm{~b}$ & 53 & 27 a cass. $e$ & \\
\hline LXXIX-LXXX & $50 \mathrm{~b}-5 \mathrm{ra}$ & $55-56$ & $28 \mathrm{a}-28 \mathrm{~b}$ & \\
\hline LXXXI & $5 \mathrm{Ib}$ & 57 & 29 a cass. $e$ & \\
\hline LXXXII & $52 \mathrm{~b}$ & 59 & $30 \mathrm{a}$ & \\
\hline LXXXIII & $47 \mathrm{a}$ & $55 \rightarrow 6 \mathrm{I}$ & 3 ra & \\
\hline LXXXIV & - & - & - & \\
\hline LXXXV & $360 a$ & - & $347 \mathrm{~b}$ & \\
\hline LXXXVI & - & - & I $27 \mathrm{~b}$ & \\
\hline LXXXVII & $328 \mathrm{~b}$ & - & $330 \mathrm{~b}$ & $35 a^{1}$ \\
\hline LXXXVIII & I $70 \mathrm{~b}$ & - & $328 \mathrm{a}$ & $\mathrm{I} \circ 9 \mathrm{a}^{\mathrm{I}} \rightarrow 2 \mathrm{Ia}^{4}$ \\
\hline LXXXIX & I Ib & 42 & \multicolumn{2}{|c|}{$2 \mathrm{Ib}$ cass., reint., $354 \mathrm{a}$} \\
\hline $\mathrm{xC}$ & I $3 a$ & 39 & \multicolumn{2}{|l|}{$20 \mathrm{a}$} \\
\hline $\mathrm{XCI}$ & 29 Ia & $3 I$ & \multicolumn{2}{|l|}{ I6a } \\
\hline $\mathrm{XCII}$ & $\mathrm{I} 6 \mathrm{~b}$ & 22 & \multicolumn{2}{|l|}{ I Ib } \\
\hline
\end{tabular}


Guliano Tanturli

\begin{tabular}{|c|c|c|c|c|}
\hline XCIII & $52 \mathrm{a}$ cass. $360 \mathrm{~b}$ & 58 & $29 \mathrm{~b}$ cass., $348 \mathrm{a}$ & \\
\hline $\mathrm{XCIV}$ & $16 a$ & 63 & $32 a$ & \\
\hline $\mathrm{XCV}$ & $\mathrm{I} 9 \mathrm{a} \rightarrow \mathrm{I} 6 \mathrm{a}^{\mathrm{I}}$ & 64 & $32 \mathrm{~b}$ & \\
\hline XCVI & I $5 \mathrm{~b}$ & 65 & $33 a$ & \\
\hline XCVII-XCVIII & $25 a-25 b$ & $70-7 \mathrm{I}$ & $35 \mathrm{~b}-36 \mathrm{a}$ & \\
\hline XCIX & $294 a$ & 73 & $37 a$ & \\
\hline C & $279^{a}$ & - & $304 a$ & $\mathrm{I} \circ 8 \mathrm{a}^{\mathrm{I}}$ \\
\hline CI & - & - & $355 \mathrm{~b}$ & \\
\hline CII-CIV & $27 a-28 a$ & $67-69$ & $34 a-35 a$ & \\
\hline $\mathrm{CV}$ & - & - & $354 \mathrm{~b}$ & \\
\hline CVI-CVII & $54 \mathrm{~b}-55 \mathrm{a}$ & $74-75$ & $37 \mathrm{~b}-38 \mathrm{a}$ & \\
\hline CVIII & $54 \mathrm{a}$ & 76 & $38 \mathrm{~b}$ & \\
\hline CIX & $56 a$ & 78 & $39 \mathrm{~b}$ & \\
\hline $\mathrm{cX}$ & $56 \mathrm{~b}$ & 77 & $39 a$ & \\
\hline CXI & $\mathrm{I} \circ 8 \mathrm{~b}$ & 80 & $40 \mathrm{~b}$ & \\
\hline CXII & Io8a & 79 & $40 \mathrm{a}$ & \\
\hline CXIII & $55 \mathrm{~b}$ & $79 \rightarrow 8 \mathrm{I}$ & 4ra & \\
\hline CXIV & $53 \mathrm{~b}$ cass., $3 \mathrm{I} 3 \mathrm{~b}$ & $80 \rightarrow 3 \mathrm{I} 3$ & $4 \mathrm{Ib}$ & \\
\hline CXV & 53 a cass., 285 a & $8 \mathrm{I}$ & $42 a$ & \\
\hline CXVI & $57 \mathrm{a}$ & 82 & $42 \mathrm{~b}$ & \\
\hline CXVII-CXVIII & $58 a-58 b$ & $83-84$ & $43 a-43 b$ & \\
\hline CXIX & $57 \mathrm{~b}$ & 87 & $45 \mathrm{a}$ & \\
\hline cXX & $35 \mathrm{Ib}$ & - & $337 \mathrm{~b}$ & $44 b^{1}$ \\
\hline CXXI & $59 \mathrm{~b}$ & 86 & $44 \mathrm{~b}$ & \\
\hline CXXII & $\begin{array}{l}65 \text { a cass. } \rightarrow \text { fra } \\
\text { le malattie, } \\
289 \text { a }\end{array}$ & 88 & $45 \mathrm{~b}$ & \\
\hline CXXIII & 6oa & - & $46 a$ & \\
\hline CXXIV & $6 o b$ & 90 & $46 b$ & \\
\hline CXXV-CXXVII & $61 a-62 a$ & $93-95$ & $47 a-48 b$ & \\
\hline CXXVIII-CXXX & $29 \mathrm{~b}-30 \mathrm{~b}$ & $97-99$ & $49^{a-50 a}$ & \\
\hline CXXXI-CXXXII & $3 \mathrm{Ia}-3 \mathrm{Ib}$ & $9^{8-99 \rightarrow \mathrm{IOO}-\mathrm{IOI}}$ & $50 \mathrm{~b}-5 \mathrm{ra}$ & \\
\hline CXXXIII & $32 a$ & 102 & $5 \mathrm{Ib}$ & \\
\hline CXXXIV & $33 a$ & 103 & $52 a$ & \\
\hline Cxxxv & $32 \mathrm{~b}$ cass., $283 \mathrm{~b}$ & - & $52 \mathrm{~b}$ & \\
\hline CXXXVI-CXXXVII & $33 \mathrm{~b}-34 \mathrm{a}$ & IO4-IOS & $53 a-53 b$ & \\
\hline CXXXVIII & $35 \mathrm{a}$ & 106 & $54 a$ & \\
\hline CXXXIX-CXL & $36 a-36 b$ & 108-109 & $55 a-55 b$ & \\
\hline CXLI-CXLII & $37 a-37 b$ & I I I - I I 2 & $56 b-57 a$ & \\
\hline CXLIII & $35 \mathrm{~b}$ & 107 & $54 \mathrm{~b}$ & \\
\hline CXLIV & $38 \mathrm{~b}$ & I I 3 & $57 \mathrm{~b}$ & \\
\hline CXLV & $48 \mathrm{a} \rightarrow$ ne' morti & I I 4 & $58 a$ & \\
\hline CXLVI-CXLVII & $39 a-39 b$ & I I 6-I I 7 & $59 a-59 b$ & \\
\hline CXLVIII-CXLIX & $40 \mathrm{~b}-4 \mathrm{Ia}$ & I 2O-I 2 I & 6ra-6rb & \\
\hline $\mathrm{CL}$ & $43 \mathrm{~b}$ & I 22 & $62 a$ & \\
\hline CLI & $42 \mathrm{a}$ cass., I69a & - & $309 a$ & $60 b^{1} \rightarrow 59 b^{1} \rightarrow 58 b^{1}$ \\
\hline CLII & $38 \mathrm{a}$ & I I 8 & $60 a$ & \\
\hline
\end{tabular}


Benedetto Varchi

\begin{tabular}{|c|c|c|c|c|}
\hline CLIII & $42 \mathrm{~b}$ cass., $3 \mathrm{I} 3 \mathrm{a}$ & I 23 & $62 \mathrm{~b}$ & \\
\hline CLIV & $4 \mathrm{Ib}$ & II 9 & Gob & \\
\hline CLV & $44 \mathrm{~b}$ & I 26 & $64 a$ & \\
\hline CLVI & $43 a$ & I 24 & $63 a$ & \\
\hline CLVII & $44 \mathrm{a}$ & I 25 & $63 \mathrm{~b}$ & \\
\hline CLVIII-CLIX & - & - & $363 a-363 b$ & \\
\hline CLX & $362 b$ & - & $359 \mathrm{a}$ & \\
\hline CLXI & - & - & c. $98 \mathrm{vb}$ & \\
\hline CLXII & $34 \mathrm{~b}$ cass., 107b & I IO & $56 a$ & \\
\hline CLXIII & $363 a$ & - & $359 \mathrm{~b}$ & \\
\hline CLXIV-CLXV & - & - & - & \\
\hline CLXVI & \multicolumn{2}{|c|}{89 a tra quei di morte 129} & $6, \mathrm{~b}$ & \\
\hline CLXVII & $69 \mathrm{~b}$ & I 30 & $66 a$ & \\
\hline CLXVIII & $68 \mathrm{a}$ & I 40 & $7 \mathrm{ra}$ & \\
\hline CLXIX & $24 \mathrm{~b}$ & 66 & $33 \mathrm{~b}$ & $7 \mathrm{Ia}^{\mathrm{I}}$ \\
\hline CLXX & $67 \mathrm{~b}$ & I $4 \mathrm{I}$ & $7 \mathrm{Ib}$ & \\
\hline CLXXI & $342 a$ & - & $317 \mathrm{~b}$ & $7 \mathrm{Ib}^{\mathrm{I}}$ \\
\hline CLXXII & - & - & - & \\
\hline CLXXIII & $92 \mathrm{a}$ cass., $284 \mathrm{a}$ & $\mathrm{I} 44 \rightarrow \mathrm{I} 43$ & $72 b$ & \\
\hline CLXXIV & $66 a$ & 146 & $74 a$ & \\
\hline CLXXV & $64 a$ & I 45 & $73 \mathrm{~b}$ & \\
\hline CLXXVI & $277 \mathrm{a}$ & - & $302 \mathrm{a}$ & $74 \mathrm{a}^{\mathrm{I}}$ \\
\hline CLXXVII-CLXXVIII & $325 a-325 b$ & - & $307 a-307 b$ & $66 a^{2}-66 a^{3}$ \\
\hline CLXXIX & $63 \mathrm{~b}$ & I6I & $8 \mathrm{Ib}$ & \\
\hline CLXXX & $8 \mathrm{ob}$ & I 47 & $74 \mathrm{~b}$ & \\
\hline CLXXXI & $62 \mathrm{~b}$ cass., I $28 \mathrm{a}$ & I 8 I & $9^{2 b}$ & \\
\hline CLXXXII & $66 \mathrm{~b}$ cass., $284 \mathrm{~b}$ & I 44 & $73 a$ & \\
\hline CLXXXIII & $75 \mathrm{a}$ & I 3 I & $66 b$ & \\
\hline CLXXXIV & $79 \mathrm{~b}$ & I 32 & $67 a$ & \\
\hline CLXXXV & $93 \mathrm{~b}$ & I35 & $68 \mathrm{~b}$ & \\
\hline CLXXXVI & $98 \mathrm{a}$ & I 37 & $69 \mathrm{~b}$ & \\
\hline CLXXXVII & $68 \mathrm{~b}$ cass. $290 \mathrm{a}$ & I 34 & $68 \mathrm{a}$ & \\
\hline CLXXXVIII & $7 \mathrm{Ia}$ & I 49 & $75 \mathrm{~b}$ & \\
\hline CLXXXIX & 286 & I 50 & $76 a$ & \\
\hline CXC & $50 a$ & I 53 & $77 \mathrm{~b}$ & \\
\hline CXCI & $9 \circ b$ & I 54 & $78 \mathrm{a}$ & \\
\hline CXCII & 9ra & I 5 I & $76 b$ & \\
\hline CXCIII & $49 \mathrm{~b}$ cass., $295 \mathrm{a}$ & I 52 & $77 \mathrm{a}$ & \\
\hline CXCIV & $74 \mathrm{~b}$ cass., $289 \mathrm{~b}$ & 159 & $8 \mathrm{ob}$ & \\
\hline CXCV & $8 \circ a$ & $\mathrm{I} 48$ & $75 a$ & \\
\hline CXCVI & $99 a$ & 20 & $\mathrm{IO} 2 \mathrm{~b}$ & \\
\hline CXCVII-CXCVIII & $99 \mathrm{~b}-\mathrm{I} 00 \mathrm{a}$ & $203-204$ & IOIb-IO2a & \\
\hline CXCIX & $76 \mathrm{a}$ cass. $107 \mathrm{a}$ & 210 & $\cos a$ & \\
\hline $\mathrm{CC}$ & 8 га & I 38 & $70 a$ & \\
\hline CCI & $93 a$ & I 89 & $94 \mathrm{~b}$ & \\
\hline CCII & IO4a & - & $308 \mathrm{a}$ & I I $4 a^{5}$ \\
\hline CCIII-CCIV & $8 \mathrm{Ib}-82 \mathrm{a}$ & I64-I65 & $83 \mathrm{~b}-84 a$ & \\
\hline
\end{tabular}


Guliano Tanturli

\begin{tabular}{|c|c|c|c|c|}
\hline $\mathrm{CCV}$ & $89 \mathrm{~b}$ & I 80 & $9 \circ \mathrm{a}$ & \\
\hline CCVI & $348 \mathrm{a}$ & & - & 33 ra \\
\hline CCVII & $88 \mathrm{~b}$ & I 74 & $89 \mathrm{~b}$ & \\
\hline CCVIII-CCXVI & $84 a-88 a$ & I66-I 73 & $84 \mathrm{~b}-88 \mathrm{~b}$ & \\
\hline CCXVII & $9 \circ \mathrm{a}$ & 178 & $89 a$ & \\
\hline CCXVIII & Io6b & I 8 I & $9 \circ \mathrm{b}$ & \\
\hline CCXIX & $\mathrm{I} O 4 \mathrm{~b}$ & 215 & $\mathrm{I} 07 \mathrm{~b}$ & \\
\hline $\operatorname{ccxx}$ & I $27 \mathrm{a}$ & - & $298 \mathrm{a}$ & $107 b^{1}$ \\
\hline CCXXI & $292 \mathrm{a}$ & - & $298 \mathrm{~b}$ & $107 b^{2}$ \\
\hline CCXXII & $343 a$ & - & 3 I $8 \mathrm{a}$ & $107 \mathrm{~b}^{3}$ \\
\hline CCXXIII & $286 a$ & 160 & 8 ra & \\
\hline CCXXIV & $67 \mathrm{a}$ cass., $278 \mathrm{a}$ & $272 ?$ cass. & $82 a$ & \\
\hline CCXXV-CCXXVI & $70 a-70 b$ & $162-163$ & $82 b-83 a$ & \\
\hline CCXXVII & $75 \mathrm{~b}$ & I $49 \rightarrow$ I 55 & $78 \mathrm{~b}$ & \\
\hline CCXXVIII & $\mathrm{I} 72 \mathrm{~b}$ & - & $305 \mathrm{~b}$ & $78 \mathrm{~b}^{\mathrm{I}}$ \\
\hline CCXXIX & $92 \mathrm{~b}$ & 156 & $79^{a}$ & \\
\hline $\operatorname{CCXXx}$ & $64 \mathrm{~b}$ cass., $290 \mathrm{~b}$ & I 57 & $79 \mathrm{~b}$ & \\
\hline CCXXXI & $278 \mathrm{~b}$ & 278 cass. & $8 \circ \mathrm{a}$ & \\
\hline CCXXXII & $279 \mathrm{~b}$ & - & $3 \circ 2 \mathrm{~b}$ & $9 \circ b^{1}$ \\
\hline CCXXXIII-CCXXXV & $296 \mathrm{~b}-97 \mathrm{~b}$ & I $82-$ I 84 & $91 a-92 a$ & \\
\hline CCXXXVI-CCXXXVI & II IоIa-IoIb & 206-207 & $103 a-103 b$ & \\
\hline CCXXXVIII & $295 \mathrm{~b}$ & 209 & $\mathrm{I} \circ 4 \mathrm{~b}$ & \\
\hline CCXXXIX & $79 \mathrm{a}$ & 196 & $98 \mathrm{a}$ & \\
\hline CCXL & $333 \mathrm{~b}$ & - & $3 \circ 8 b$ & $\mathrm{I} I 4 \mathrm{a}^{3}$ \\
\hline CCXLI & Io5a & $218 ? \rightarrow 220$ & I I Oa & \\
\hline CCXLII & $36 \mathrm{Ib}$ & - & $352 \mathrm{~b}$ & \\
\hline CCXLIII & $358 \mathrm{a}$ & - & $35 \mathrm{Ib}$ & \\
\hline CCXLIV & $95 \mathrm{a}$ & $20 \mathrm{I}$ & Ioob & \\
\hline CCXLV & $94 \mathrm{~b}$ & 200 & I o०a & \\
\hline CCXXLVI-CCXLVII & $274 a-274 b$ & - & I I $2 a-$ I I $2 b$ & \\
\hline CCXLVIII & $329 a$ & - & 3 iоa & I I $2 b^{I}$ \\
\hline CCXLIX & $338 \mathrm{~b}$ & - & 3 I $4 a$ & I I $2 b^{2}$ \\
\hline CCL-CCLI & $330 \mathrm{a}-33 \mathrm{ob}$ & - & 3 I Ia-3 I Ib & I I $2 b^{3}-$ I I $2 b^{4}$ \\
\hline CCLII & $329 \mathrm{~b}$ & - & $3 \mathrm{Iob}$ & I I $2 b^{5}$ \\
\hline CCLIII-CCLIV & $33 \mathrm{Ia}-33 \mathrm{Ib}$ & - & 3 I $2 a-3$ I $2 b$ & I I $2 b^{6}$ - I I $2 b^{7}$ \\
\hline CCLV & $345 \mathrm{~b}$ & - & $3 \mathrm{I} 3 \mathrm{~b}$ & I I $2 b^{8}$ \\
\hline CCLVI & $346 a$ & - & $329 a$ & I I $2 b^{10}$ \\
\hline CCLVII & $332 a$ & - & $3 \mathrm{I} 3 \mathrm{a}$ & I I $2 b^{9}$ \\
\hline CCLVIII & $328 \mathrm{a}$ & - & $317 a$ & I I $2 b^{12}$ \\
\hline CCLIX & $338 \mathrm{a}$ & - & 3 I $4 b$ & I I $2 b^{\text {II }}$ \\
\hline CCLX & $342 \mathrm{~b}$ & - & $327 \mathrm{~b}$ & I I $2 b^{13}$ \\
\hline CCLXI & $34 \mathrm{ra}$ & - & $3 \mathrm{igb}$ & \\
\hline CCLXII-CCLXIII & $26 a-26 b$ & I93-I94 & $9^{6 b-97 a}$ & \\
\hline CCLXIV & $83 a$ & I98 & $99 \mathrm{~b}$ & \\
\hline CCLXV & $343 a$ & - & 3 iqa & $99 a^{1}$ \\
\hline CCLXVI & Io6a & $217 \rightarrow 219$ & $\mathrm{I} \circ 9 \mathrm{~b}$ & $99 \mathrm{a}^{2}$ \\
\hline CCLXVII & I $28 \mathrm{~b}$ & - ultimo & I I 4 b & \\
\hline
\end{tabular}


Benedetto Varchi

\begin{tabular}{|c|c|c|c|c|}
\hline CCLXVIII & $292 b$ & - & $328 b$ & $109 a^{2} \rightarrow 66 a^{1}$ \\
\hline CCLXIX & $287 a$ & $2 \mathrm{I} 8 \rightarrow 2 \mathrm{I} 7$ & $\mathrm{I} \circ 8 \mathrm{~b}$ & \\
\hline CCLXX & IO2a & $200 \longrightarrow 2 \mathrm{I} 7$ & Ioga & \\
\hline CCLXXI & $83 \mathrm{~b}$ & I97 & $98 b$ & \\
\hline CCLXXII & - & - & - & \\
\hline CCLXXIII & $333 a$ & - & $3 \mathrm{I} 8 \mathrm{a}$ & I I I $b^{I}$ \\
\hline CCLXXIV & $\mathrm{I} O 2 \mathrm{~b}$ & I 38 & $70 \mathrm{~b}$ & \\
\hline CCLXXV-CCLXXIX & - & - & - & \\
\hline CCLXXX & $65 \mathrm{~b}$ cass., 3 I $2 \mathrm{~b}$ & I 88 & $94 a$ & \\
\hline CCLXXXI & $7 \mathrm{Ib}$ & I 87 & $93 \mathrm{~b}$ & \\
\hline CCLXXXII-CCLXXXIII & II $72 \mathrm{a}-72 \mathrm{~b}$ & I90-I9I & $95 \mathrm{a}-95 \mathrm{~b}$ & \\
\hline CCLXXXIV & $73 \mathrm{~b}$ & 192 & $9^{6 \mathrm{a}}$ & \\
\hline CCLXXXV & $362 a$ & - & $353 \mathrm{~b}$ & \\
\hline CCLXXXVI & $74 \mathrm{a}$ & 2 I 2 & ro6a & \\
\hline CCLXXXVII & Iо3a & 3 & Io6b & \\
\hline CCLXXXVIII & $73 a$ & 2 I I & $105 b$ & \\
\hline CCLXXXIX & $\mathrm{IO} 3 \mathrm{~b}$ & 214 & I07a & \\
\hline $\mathrm{CCXC}$ & $82 \mathrm{~b}$ & I99 & $99 \mathrm{~b}$ & \\
\hline $\mathrm{CCXCI}$ & $\mathrm{I} 69 \mathrm{~b}$ & - & $309 \mathrm{~b}$ & $66 \mathrm{~b}$ cass. \\
\hline CCXCII & Ioob & 208 & IO4a & \\
\hline CCXCIII & I $26 \mathrm{~b}$ & - & I I I a & \\
\hline CCXCIV & $\mathrm{I} 7 \mathrm{Ib}$ & - & $306 \mathrm{~b}$ & I I $3 b^{I}$ \\
\hline $\mathrm{CCXCV}$ & I $25 \mathrm{~b}$ cass. I $26 \mathrm{a}$ & - & I I $4 \mathrm{a}$ & \\
\hline CCXCVI & I7 Ia & - & $306 a$ & I I $4 a^{I}$ \\
\hline CCXCVII & $354 a$ & - & $340 \mathrm{a}$ & I I $4 a^{4}$ \\
\hline CCXCVIII & $345 \mathrm{a}$ & - & $325 b$ & I I $4 \mathrm{a}^{2}$ \\
\hline CCXCIX & I $25 \mathrm{a}$ & - & I I ob & \\
\hline $\mathrm{CCC}$ & $77 \mathrm{a}$ & 195 & $97 \mathrm{~b}$ & \\
\hline CCCI & - & - & $364 a$ & \\
\hline CCCII-CCCX & - & - & - & \\
\hline CCCXI & - & - & c. $98 \mathrm{vb}$ & \\
\hline CCCXII & - & - & - & \\
\hline CCCXIII & $296 b$ & 230 & I I 5 a & I I $4 a^{I}$ \\
\hline CCCXIV-CCCXVII & $297 a-298 b$ & $23 I-234$ & I I $5 \mathrm{~b}-$ I I $7 \mathrm{a}$ & \\
\hline CCCXVIII & $300 a$ & 236 & I I $8 \mathrm{a}$ & \\
\hline CCCXIX & $299 a$ & 235 & I I $7 \mathrm{~b}$ & \\
\hline $\operatorname{cccxx}$ & $299 \mathrm{~b}$ & 237 & I I $8 b$ & \\
\hline CCCXXI & $355 a$ & - & $34 \mathrm{ra}$ & I I $4 \mathrm{a}^{2}$ \\
\hline CCCXII & - & - & $344 a$ & \\
\hline CCCXXIII-CCCXXIV - & - & - & $345 \mathrm{~b}-346 \mathrm{a}$ & \\
\hline CCCXXV & $359 a$ & - & $346 b$ & \\
\hline CCCXXVI & $363 \mathrm{~b}$ & - & $356 a$ & \\
\hline CCCXXVII & $364 \mathrm{~b}$ & - & $356 \mathrm{~b}$ & \\
\hline CCCXXVIII & $364 a$ & - & $357 \mathrm{~b}$ & \\
\hline CCCXXIX & $357 \mathrm{~b}$ & - & $35 \mathrm{ra}$ & \\
\hline $\operatorname{CCCXXX}$ & $358 \mathrm{~b}$ & - & $352 a$ & \\
\hline CCCXXXI & $\mathrm{I} 05 \mathrm{~b}$ & 216 & Io8a & \\
\hline
\end{tabular}


Guliano Tanturli

\begin{tabular}{|c|c|c|c|}
\hline CCCXXXII & $301 \mathrm{a}$ & 237 & I I 9 a \\
\hline \multicolumn{2}{|c|}{ CCCXXXIII-CCCXXXV $302 \mathrm{~b}-3 \circ 3 \mathrm{~b}$} & $238-240$ & I I $9 \mathrm{~b}-\mathrm{I} 2 \mathrm{Ob}$ \\
\hline CCCXXXVI & $306 a$ & $24 \mathrm{I}$ & I 2 I a \\
\hline \multicolumn{2}{|c|}{ CCCXXXVII- CCCXXXVIII 304a-304b } & $242-243$ & I 2 I b-I $22 \mathrm{a}$ \\
\hline CCCXXXIX & $307 a$ & 94 & $48 \mathrm{~b}$ \\
\hline CCCXL & $306 \mathrm{~b}$ & 245 & I $23 \mathrm{a}$ \\
\hline CCCXLI & $308 \mathrm{~b}$ & 246 & I $23 \mathrm{~b}$ \\
\hline CCCXLII & $308 \mathrm{a}$ & 244 & I $22 \mathrm{~b}$ \\
\hline CCCXLIII & $302 \mathrm{a}$ & 247 & I $24 \mathrm{a}$ \\
\hline CCCXLIV & $40 \mathrm{a}$ & II 5 & $58 \mathrm{~b}$ \\
\hline CCCXLV & $30 \mathrm{Ib}$ & 248 & I $24 \mathrm{~b}$ \\
\hline CCCXLVI & $307 \mathrm{~b}$ & 249 & I $25 \mathrm{a}$ \\
\hline \multicolumn{2}{|c|}{ CCCXLVII-CCCXLVIII $305 \mathrm{a}-305 \mathrm{~b}$} & $250-25 \mathrm{I}$ & I $25 \mathrm{~b}-\mathrm{I} 26 \mathrm{a}$ \\
\hline CCCXLIX-CCCL & $309 a-309 b$ & $252-253$ & I $26 \mathrm{~b}-\mathrm{I} 27 \mathrm{a}$ \\
\hline CCCLI & - & - & - \\
\hline \multicolumn{2}{|c|}{ CCCLII-CCCLXIV I I I a- I I 7 a } & $254-266$ & I $28 \mathrm{a}-\mathrm{I} 34 \mathrm{a}$ \\
\hline CCCLXV & $\mathrm{I} 2 \mathrm{Ob}$ & 267 & I $34 \mathrm{~b}$ \\
\hline CCCLXVI & I $22 \mathrm{a}$ & - & I $36 a$ \\
\hline CCCLXVII-CCCLXIX I & I I $8 a-$ I I $9 a$ & $270-272$ & I $36 \mathrm{~b}-137 \mathrm{~b}$ \\
\hline CCCLXX & I 2 I a & 275 & I $39 \mathrm{a}$ \\
\hline CCCLXXI & I $20 \mathrm{a}$ & 273 & I $38 \mathrm{a}$ \\
\hline CCCLXXII & I I gb & 274 & I $38 b$ \\
\hline CCCLXXIII & $123 \mathrm{a}$ & 277 & I $40 \mathrm{a}$ \\
\hline CCCLXXIV & I I 7 b & $276 \rightarrow 277$ & $\mathrm{I} 4 \mathrm{Ob}$ \\
\hline CCCLXXV & $\mathrm{I} 2 \mathrm{Ib}$ & 278 & I $4 \mathrm{Ia}$ \\
\hline CCCLXXVI & $123 \mathrm{~b}$ & 279 & I $4 \mathrm{Ib}$ \\
\hline CCCLXXVII- CCCLXXI & IVIIII $28 \mathrm{~b}-29 \mathrm{a}$ & $268-269$ & I $35 \mathrm{a}-\mathrm{I} 35 \mathrm{~b}$ cass.?* \\
\hline
\end{tabular}

*[Sulla pagina c'è un tratto di penna che taglia in diagonale il cCcLXxvII e tocca l'incipit del CCCLXXVIII. Per solito i sonetti da espungere sono sbarrati almeno con due diagonali incrociate.]

$\begin{array}{llll}\text { CCCLXXIX } & - & - & 360 \mathrm{~b} \\ \text { CCCLXXX } & - & - & \text { c. IIrb } \\ \text { CCCLXXXI } & - & - & \text { VIra } \\ \text { CCCLXXXII } & - & - & \text { VIvb } \\ \text { CCCLXXXIII } & - & - & \text { IVra } \\ \text { CCCLXXXIV } & - & - & \text { IIva } \\ \text { CCCLXXXV } & - & - & \text { IIIra } \\ \text { CCCLXXXVI } & - & - & 95 \text { ra } \\ \text { CCCLXXXVII } & - & - & \text { VIrb } \\ \text { CCCLXXXVIII } & - & - & 95 \text { rb } \\ \text { CCCLXXXIX } & - & - & \text { IVrb } \\ \text { CCCXC } & - & - & 98 \mathrm{ra} \\ \text { CCCXCI } & - & - & \text { VIIIV } \\ \text { CCCXCII-CCCXCIII } & - & - & \text { pp. } 64 \mathrm{~b}-65 \mathrm{a} \\ \text { CCCXCIV } & - & - & \text { c. IIIrb } \\ \text { CCCXCV } & - & - & \text { IIvb }\end{array}$




\section{Benedetto Varchi}

\begin{tabular}{|c|c|c|c|}
\hline CCCXCVI & - & - & viva \\
\hline CCCXCVII-CCCCXVI & - & - & - \\
\hline CCCCXVII & - & - & IV \\
\hline CCCCXVIII & - & - & Ir \\
\hline CCCCXIX-CCCCLXIII & - & - & - \\
\hline CCCCLXIV & 317 & I 27 & p. 65a incipit cass., $30 \mathrm{Ib}$ \\
\hline CCCCLXV-CCCCXCI & - & - & - \\
\hline CCCCXCII & $47 \mathrm{~b}$ & 62 & $3 \mathrm{Ib}$ cass. \\
\hline CCCCXCIII-CCCCXCV & $V-$ & - & - \\
\hline CCCCXCVI & I $22 \mathrm{~b}$ & 276 & I $39 \mathrm{~b}$ cass. \\
\hline CCCCXCVII-DXXXIV & - & - & - \\
\hline t57 & $N$ & $N^{I}$ & $M^{I}$ \\
\hline I-III & I 29-I 3 I & $280-282$ & I 43-I 45 \\
\hline IV & $\begin{array}{l}\text { I } 60 \text { cogli altri al } \\
\text { medesimo }\end{array}$ & 283 & 146 \\
\hline $\mathrm{V}$ & 132 & 284 & I 47 \\
\hline VI & I6 I cogli altri & 285 & I 48 \\
\hline VII-IX & I33-I35 & $286-288$ & I 49-I 5 I \\
\hline $\mathrm{X}$-XII & I $37-$ I 39 & $289-29 \mathrm{I}$ & I $52-I 54$ \\
\hline XIII & I 50 & - & 165 \\
\hline XIV & I 5 I & 303 & 166 \\
\hline $\mathrm{xV}$ & I 52 & - & 167 \\
\hline XVI & 346b prop. cass., 3 & $350-$ & $329 \mathrm{~b}$ prop. cass., $334 \mathrm{I}^{6} 9^{\mathrm{I}} \rightarrow \mathrm{I} 68^{\mathrm{I}}$ \\
\hline XVII & 3 I I & 305 & I68 \\
\hline XVIII & 168 & - & 169 \\
\hline XIX & - & - & c. $\mathrm{VV}$ \\
\hline $\mathrm{XX}-\mathrm{XXI}$ & I $53-\mathrm{I} 54$ & $306-307$ & $\mathrm{I} 7 \mathrm{I}-\mathrm{I} 72$ \\
\hline XXII & 276 & - & 170 \\
\hline XXIII & $347 \mathrm{~b}$ prop. cass. 3 & $53-$ & $333 \mathrm{~b}$ prop. cass., 338 \\
\hline XXIV & $\begin{array}{l}352 \mathrm{a} \text { prop. } 354 \mathrm{~b} r \\
\text { con reciproco richian }\end{array}$ & risp. - & 339 \\
\hline $\mathrm{xxV}$ & - & - & c. VIIr \\
\hline XXVI & I 57 & 308 & 173 \\
\hline XXVII & I 59 & 3 I I & 176 \\
\hline XXVIII & - & - & 367 \\
\hline XXIX & - & - & c. 95 va prop. c. IIra risp. \\
\hline $\mathrm{xxx}$ & - & - & c. VIIIr \\
\hline XXXI & - & - & p. 370 solo prop. \\
\hline XXXII & - & - & - \\
\hline XXXIII & 355b solo prop. & - & $340 \mathrm{~b}$ solo prop. $\quad 68 \mathrm{~b}^{\mathrm{I}}$ cass. \\
\hline XXXIV & $95 \mathrm{~b}$ solo prop. & 202 & ror a solo prop. \\
\hline XXXV & $77 \mathrm{~b}$ prop. cass., I 6 & 663 I 2 & I 77 \\
\hline XXXVI & $78 \mathrm{~b}$ solo prop. & 133 & $\begin{array}{l}67 \mathrm{~b} \text { prop. con richiamo alla risp., } 353 \mathrm{a} \\
\text { risp. }\end{array}$ \\
\hline XXXVII & 136 & 294 & I 57 \\
\hline
\end{tabular}


Guliano Tanturli

\begin{tabular}{|c|c|c|c|}
\hline XXXVIII & $\mathrm{I} 48$ & 295 & I 58 \\
\hline XXXIX & I 43 & 293 & I 56 \\
\hline $\mathrm{XL}$ & I 39 & 292 & I 55 \\
\hline XLI & \multicolumn{3}{|c|}{ 59а prop., I 70 a risp. } \\
\hline XLII & - & - & - \\
\hline XLIII & I 49 & 298 & 160 \\
\hline XLIV & I 56 & 309 & 174 \\
\hline $\mathrm{XLV}$ & I 45 & 316 & I 82 \\
\hline XLVI & 288 & - & I 83 \\
\hline XLVII & \multicolumn{2}{|c|}{283 a prop, 36 I a risp. - } & I I 3 b prop. cass., 343 \\
\hline XLVIII & 165 & 315 & I 80 \\
\hline XLIX & \multicolumn{2}{|c|}{282,294 b prop.cass. - } & I 8 I \\
\hline $\mathrm{L}$ & - & - & - \\
\hline LI & 9ib solo prop. & I 42 solo prop. & $\begin{array}{l}72 \mathrm{a} \text { prop. } 35 \text { ob risp con } \\
\text { richiamo reciproco }\end{array}$ \\
\hline LII & - & - & 349 \\
\hline LIII & I 58 & 319 & I75 \\
\hline LIV & - & - & - \\
\hline LV & 287b solo prop. & - & $\begin{array}{l}\text { I I Ib prop. cass., I7 } \\
336\end{array}$ \\
\hline LVI & - & - & 362 \\
\hline LVII-LVIII & I 46-I 47 & $298-299$ & $16 I-I 62$ \\
\hline LIX-LX & I 40-I 4I & $300-301$ & $163-164$ \\
\hline LXI & \multicolumn{2}{|c|}{$348 \mathrm{~b}$ prop. $357 \mathrm{a}$ risp - } & $33 \mathrm{Ib}$ prop. $35 \mathrm{ob}$ risp. \\
\hline LXII & I64 & $3 \mathrm{I} 4$ & 179 \\
\hline LXIII & \multicolumn{2}{|c|}{ I $27 \mathrm{~b}$ prop. $352 \mathrm{~b}$ risp. - } & $\begin{array}{l}\text { I } 13 \text { a prop. cass. I60 } \\
342\end{array}$ \\
\hline LXIV & - & - & $344 \mathrm{~b}-345 \mathrm{a}$ \\
\hline LXV & - & - & c. IVV \\
\hline LXVI & - & - & - \\
\hline LXVII & - & - & $9^{8 \mathrm{rb}}$ \\
\hline LXIX-LXXIV & - & - & - \\
\hline LXXV & I 55 & $3 \mathrm{I} 3$ & p. 178 \\
\hline LXXVI & 365 & - & 357 a prop. $360 a$ risp. \\
\hline LXXVII & 162 & 318 & I 84 \\
\hline LXXVIII & \multicolumn{2}{|c|}{ 288b solo prop. I67 3 I9 } & I 85 \\
\hline LXXIX & 163 & - & I 86 \\
\hline LXXX & - & - & - \\
\hline LXXXI & $23 I$ & $3 \mathrm{I} 3$ & $23 I$ \\
\hline LXXXII & 223 & 374 & 264 \\
\hline LXXXIII-LXXXVI & $200-203$ & $345-348$ & $224-227$ \\
\hline LXXXVII & 235 & 349 & 228 \\
\hline LXXXVIII & 229 & 362 & 242 \\
\hline LXXXIX-XCI & $240-242$ & $363-365$ & $243-245$ \\
\hline XCII-XCIII & 2IO-2 I I & $366-367$ & $246-247$ \\
\hline $\mathrm{XCIV}$ & $236 \rightarrow 26 \mathrm{I}^{\mathrm{I}}$ & 396 & 290 \\
\hline XCV & - & - & 369 \\
\hline
\end{tabular}




\section{Benedetto VARchi}

\begin{tabular}{|c|c|c|c|}
\hline XCVI & - & - & 368 \\
\hline XCVII & $26 \mathrm{I}$ & 397 & $29 \mathrm{I}$ \\
\hline XCVIII & $272 \rightarrow 26 \mathrm{I}^{3}$ & $39^{8}$ & 292 \\
\hline XCIX & - & - & c. IIIV \\
\hline C & - & - & - \\
\hline CI & - & - & c. $\mathrm{VV}$ \\
\hline CII & 2 I 2 cass., 3 I 8 & - & 299 \\
\hline CIII & $2 \mathrm{I} 3$ & $37 \mathrm{I}$ & 262 \\
\hline $\mathrm{CIV}$ & \multicolumn{2}{|c|}{$3 \mathrm{I} 5$ cass. $\rightarrow 2 \mathrm{I} 2 / 2 \mathrm{I}^{*}-$} & 263 \\
\hline
\end{tabular}

* [A p. 3 I 5 in alto a destra è scritto: «a carte 2 I 2», a sinistra: «a 2I 3»; nel margine inferiore di p. 2 I 2 sotto p. $2^{a}$ CIII è scritto e cassato: «qui s'ha a scrivere il sonetto, che comincia Arsi con dura, insopportabil sorte colle risposte, ch'è in questo a carte 31 5 ", tutto di mano del Varchi.]

$\begin{array}{llll}\text { CV-CVI } & \text { I 77-I78 } & 324-325 & \text { I93-I94 } \\ \text { CVII } & - & - & - \\ \text { CVIII-CX } & \text { I } 74-176 & 32 \text { I-323 } & \text { I90-I92 } \\ \text { CXI } & 230 & 365 & 255 \\ \text { CXII } & 244 & 366 & 256 \\ \text { CXIII } & 27 \text { I } \rightarrow 244 & 367 & 257 \\ \text { CXIV } & - & - & - \\ \text { CXV } & 232 & 354 & 232 \\ \text { CXVI } & 243 & - & 233 \\ \text { CXVII* } & - & - & -\end{array}$

*[C'è qui un incidente nella successione e numerazione di Benedetto Varchi, Opere, pp. 947-48: alla coppia CXVI, p. I 2 I di t57, deve succedere, ivi, p. I 22, la proposta del medesimo Simone della Volta, L'altera e bella donna cui tanto ama e la risposta Tai furon l'opre sue, tanta è la fama, cui si assegna il numero CXVII (non cXvIII), quindi, p. I 23, la proposta di Carlo Strozzi Varchi s'ad alcun mai pianto e dolore e la risposta Ben conosco il mio folle e vano errore, CXVIII (non CXVII), infine, p. I 24, la proposta del medesimo L'erto sentiero, onde si poggia al monte con la risposta Carlo che con gran passi a fuggir l'onte, cxix.]

$\begin{array}{llll}\text { CXVIII } & 207 & 360 & 250 \\ \text { CXIX } & 206 & 36 \mathrm{I} & 25 \mathrm{I} \\ \text { CXX } & 205 & 362 & 252 \\ \text { CXXI } & 204 & 363 & 253 \\ \text { CXXII } & 260 & 387 & 28 \mathrm{I} \\ \text { CXXIII } & - & - & - \\ \text { CXXIV } & 267 & 346 & 2 \mathrm{I} 5 \\ \text { CXXV } & 262 & 392 & 286 \\ \text { CXXVI } & 263 & 389 & 283 \\ \text { CXXVII } & 258 & 388 & 282 \\ \text { CXXVIII } & 197 & 343 & 2 \mathrm{I} 2 \\ \text { CXXIX } & 208 & 368 & 248 \\ \text { CXXX } & 259 & 393 & 287 \\ \text { CXXXI-CXXXV } & 247-25 \mathrm{I} & 377-38 \mathrm{I} & 267-27 \mathrm{I} \\ \text { CXXXVI } & 3 \mathrm{I} 9 & - & 272 \\ \text { CXXXVII } & 32 \mathrm{I} & - & 273\end{array}$


Guliano Tanturli

\begin{tabular}{|c|c|c|c|c|}
\hline CXXXVIII & 320 & - & 274 & \\
\hline CXXXIX & 273 & 382 & 275 & \\
\hline CXL & 322 & - & 276 & \\
\hline CXLI & - & - & - & \\
\hline CXLII & 252 & 383 & 277 & \\
\hline CXLIII & 255 & 386 & 278 & \\
\hline CXLIV-CXLV & $253-254$ & $384-386$ & $279-280$ & \\
\hline CXLVI-CXLVII & $326-327$ & - & $315-316$ & $280^{\mathrm{I}}-280^{2}$ \\
\hline CXLVIII-CXLIX & - & - & - & \\
\hline CL & 173 & 320 & 189 & \\
\hline CLI-CLII & I 86-I 87 & $333-334$ & $202-203$ & \\
\hline CLIII & $19^{2}$ & 338 & 207 & \\
\hline CLIV & I 8 I & 328 & 197 & \\
\hline CLV & I 83 & 329 & I98 & \\
\hline CLVI-CLVIII & I89-I9I & $335-337$ & 204-206 & \\
\hline CLIX-CLXII & $193-196$ & $339-342$ & 208-2 I I & \\
\hline CLXIII-CLIV & - & - & - & \\
\hline CLXV & 217 & 344 & $2 \mathrm{I} 3$ & \\
\hline CLXVI & I 88 cass. 324 & - & 295 & \\
\hline CLXVII & I 82 & 345 & $2 \mathrm{I} 4$ & \\
\hline CLXVIII & I99 & 369 & 260 & \\
\hline CLXIX & 109 & - & 259 & \\
\hline CLXX & I 80 & 326 & 195 & \\
\hline CLXXI & I 79 & 327 & 196 & \\
\hline CLXXII & 245 & 375 & 265 & \\
\hline CLXXIII & - & - & $36 \mathrm{I}$ & \\
\hline CLXXIV & I 24 & - & 254 & \\
\hline CLXXV & - & - & 365 & \\
\hline CLXXVI & - & - & - & \\
\hline CLXXVII-CLXXVIII & I $84-$ I 85 & $330-33 \mathrm{I}$ & $199-200$ & \\
\hline CLXXIX & I IO & - & $20 \mathrm{I}$ & \\
\hline CLXXX & 264 & 368 & 258 & \\
\hline CLXXXI & 216 & 349 & $2 \mathrm{I} 8$ & \\
\hline CLXXXII & 215 & 348 & $2 \mathrm{I} 7$ & \\
\hline CLXXXIII-CLXXXV & 2 I $8-220$ & $340-342$ & $219-22 I$ & \\
\hline CLXXXVI & $22 \mathrm{I}$ & 373 & 222 & \\
\hline CLXXXVII & 222 & 344 & 223 & \\
\hline CLXXXVIII & 226 & 350 & 229 & \\
\hline CLXXXIX & 323 & - & 300 & $229^{\mathrm{I}}$ \\
\hline $\mathrm{CXC}$ & 227 & $35 \mathrm{I}$ & 230 & \\
\hline CXCI & 233 & 356 & 235 & \\
\hline CXCII & 270 & - & 236 & \\
\hline CXCIII & 234 & 355 & 234 & \\
\hline CXCIV-CXCVI & $237-239$ & $359-36 I$ & $239-24 \mathrm{I}$ & \\
\hline CXCVII & 209 & 369 & 249 & \\
\hline CXCVIII & I98 & 370 & 26I & \\
\hline CXCIX & 225 & 357 & 237 & \\
\hline
\end{tabular}




\section{Benedetto Varchi}

\begin{tabular}{|c|c|c|c|c|}
\hline CC & 224 & 358 & 238 & \\
\hline CCI & 246 & 376 & 266 & \\
\hline CCII & - & - & 358 & \\
\hline CCIII & - & - & - & \\
\hline CCIV & - & - & 366 & \\
\hline CCV-CCVIII & - & - & - & \\
\hline CCIX-CCX & $265-266$ & $394-395$ & $288-289$ & \\
\hline CCXI-CCXII & $256-257$ & $390-391$ & $284-285$ & \\
\hline CCXIII-CCXIV & $268-269$ & $399-400$ & $293-294$ & \\
\hline CCXV & 344 & - & 326 & $294^{I}$ \\
\hline CCXVI & 349 & - & 335 & $295^{\mathrm{I}}$ \\
\hline CCXVII-CCXIX & - & - & - & \\
\hline
\end{tabular}

INCIPIT IN ORDINE ALFABETICO DEI SONETTI PRESENTI

NEI CODICI E NON STAMPATI IN t5sO t57

\begin{tabular}{|c|c|c|c|c|}
\hline & $N$ & $N^{I}$ & $M$ & $M^{I}$ \\
\hline $\begin{array}{l}\text { Altera e vaga e pura e chiara Petra } \\
\text { Ben m'è caro e giocondo, ma nel vero con } \\
\text { la prop. di Benedetto Cipello }\end{array}$ & p. 24 a cass. & - & - & \\
\hline $\begin{array}{l}\text { Quell' aura onde Helicona il vostro } \\
\text { altero }\end{array}$ & $2 \mathrm{I} 4$ & 347 & 2 I 6 & $295^{3}$ \\
\hline $\begin{array}{l}\text { Caro Cipello mio, se cosi vero } \\
\text { seconda Risp. a B. Cipello }\end{array}$ & & & & \\
\hline Quell'aura onde Helicona & $296 a$ & - & $327 a$ & $2 \mathrm{I}^{\mathrm{I}} \rightarrow 295^{3}$ \\
\hline $\begin{array}{l}\text { CASA, ove i miglior frutti e' più bei fiori } \\
\text { Ch'a voi, Stufa gentil, ch'a paro a paro } \\
\text { con la prop. di Giulio della Stufa }\end{array}$ & 69a cass. & - & - & \\
\hline Varchi immortal, che quanto sete raro & - & - & c. VIIV & \\
\hline $\begin{array}{l}\text { Deh, dimmi, Amor, se quelle ardenti } \\
\text { stelle }\end{array}$ & $23 \mathrm{~b}$ cass. & - & - & \\
\hline Donna, ch'a singular bellezza bavete & $48 \mathrm{~b}$ & - & - & \\
\hline Furia crudel, che dal più basso loco & $23 a$ & 54 & $27 \mathrm{~b}$ cass & \\
\hline Già so io ben ch'un amoroso ingegno & 98 a cass. & - & - & \\
\hline $\begin{array}{l}\text { Giovambatista e Giovanni, che quella } \\
\text { Guglia, c'habbia di me nel vostro core } \\
\text { Con la proposta del Guglia }\end{array}$ & $76 \mathrm{~b}$ cass. & - & - & \\
\hline Varchi, l'invitto vostro alto valore & 228 & 352 & - & \\
\hline $\begin{array}{l}\text { Hor che due volte cinque lustri intorno } \\
\text { L'arbor gentil che forte amai molti anni } \\
\text { Con la prop. di Lucantonio Ridolfi }\end{array}$ & $28 \mathrm{Ib}$ & 403 & $297 \mathrm{~b}$ & \\
\hline $\begin{array}{l}\text { Parmi, Varchi, ogni di più di mille anni } \\
\text { Nell'altrui dolci rime i tristi affanni } \\
\text { seconda Risp. a L. Ridolfi }\end{array}$ & 280 & - & 296 & \\
\hline Parmi, Varchi ogni di & 28 Ia & 402 & $297 a$ & \\
\hline $\begin{array}{l}\text { Nuova del nostro mar vaga sirena } \\
\text { Or ... vedi Hor ... } \\
\text { Parmi, Varchi, ogni di } \\
\text { prop. di Lucantonio Ridolfi a } \\
\text { L'arbor gentil che forte }\end{array}$ & $78 \mathrm{~b}$ & I 86 & $93 a$ cass. & \\
\hline Più non mi par, Bettin del dritto fore & 63 a cass. & - & - & \\
\hline
\end{tabular}


Guliano Tanturli

Quanto dianzi alta, oimé, chiara e gen- 3 I 6 a

$30 \mathrm{Ib}, \quad 64 \mathrm{~b}$

tile

incipit cass.

Quell'aura onde Helicona

prop. a

Ben m'è caro e giocondo

S'a si rara beltà voglie e costumi 9b cass.

Sacro, ben nato, avventuroso fiore $\quad 300$

$\begin{array}{ll}\text { Se da grandine o pioggia o sole o vento } & \mathrm{I} 9 \mathrm{~b} \text { cass. } \\ \mathrm{Se} \text { da leggiadra impresa alto valore } & 94 \mathrm{a} \text { cass. }\end{array}$

Urban mentre che voi d'ombre e di fumi 96a

Varchi, l'invitto vostro

prop. a

Guglia, c'abbia di me

Varchi immortal, che quanto

prop. a

Ch'a Voi, Stufa gentil 